Mon. Not. R. Astron. Soc. 000,1 1-?? (2016) Printed 16 January $2018 \quad$ (MN LATEX style file v2.2)

\title{
New Cataclysmic Variables and other Exotic Binaries in the Globular Cluster 47 Tucanae ${ }^{\star}$
}

\author{
L. E. Rivera-Sandoval ${ }^{1} \dagger$, M. van den Berg $^{2,1}$, C. O. Heinke ${ }^{3,4}$, H. N. Cohn ${ }^{5}$, \\ P. M. Lugger ${ }^{5}$, J. Anderson ${ }^{6}$, A. M. Cool ${ }^{7}$, P. D. Edmonds ${ }^{2}$, R. Wijnands ${ }^{1}$, \\ N. Ivanova ${ }^{3}$ and J. E. Grindlay ${ }^{2}$ \\ 1 Anton Pannekoek Institute for Astronomy, University of Amsterdam, Science Park 904, 1098 XH Amsterdam, The Netherlands \\ 2 Harvard-Smithsonian Center for Astrophysics, 60 Garden Street, Cambridge, MA 02138, USA \\ 3 Department of Physics, University of Alberta, CCIS 4-183, Edmonton, AB 435 T6G 2E1, Canada \\ ${ }^{4}$ Max Planck Institute for Radio Astronomy, Auf dem Hugel 69, 53121 Bonn, Germany \\ 5 Department of Astronomy, Indiana University, 727 E. Third St, Bloomington, IN 47405, USA \\ 6 Space Telescope Science Institute, 3700 San Martin Drive, Baltimore, MD 21218, USA \\ 7 Department of Physics and Astronomy, San Francisco State University, 1600 Holloway Avenue, San Francisco, CA 94132, USA
}

16 January 2018

\begin{abstract}
We present 22 new ( +3 confirmed) cataclysmic variables $(\mathrm{CVs})$ in the non corecollapsed globular cluster 47 Tucanae (47 Tuc). The total number of CVs in the cluster is now 43 , the largest sample in any globular cluster so far. For the identifications we used near-ultraviolet (NUV) and optical images from the Hubble Space Telescope, in combination with X-ray results from the Chandra X-ray Observatory. This allowed us to build the deepest NUV CV luminosity function of the cluster to date. We found that the CVs in 47 Tuc are more concentrated towards the cluster center than the main sequence turnoff stars. We compared our results to the CV populations of the core-collapsed globular clusters NGC 6397 and NGC 6752 . We found that 47 Tuc has fewer bright CVs per unit mass than those two other clusters. That suggests that dynamical interactions in core-collapsed clusters play a major role creating new CVs. In 47 Tuc, the CV population is probably dominated by primordial and old dynamically formed systems. We estimated that the CVs in 47 Tuc have total masses of $\sim 1.4 M_{\odot}$. We also found that the X-ray luminosity function of the CVs in the three clusters is bimodal. Additionally, we discuss a possible double degenerate system and an intriguing/unclassified object. Finally, we present four systems that could be millisecond pulsar companions given their X-ray and NUV/optical colors. For one of them we present very strong evidence for being an ablated companion. The other three could be CO- or He-WDs.
\end{abstract}

Key words: Binaries - Cataclysmic variables. Globular clusters - 47 Tucanae

\section{INTRODUCTION}

Cataclysmic variables (CVs) are binary stars that harbor a white dwarf (WD) that accretes from a low-mass companion. These systems have orbital periods between $\sim 75$ mins and $10 \mathrm{~h}$, and the overall distribution of $\mathrm{CV}$ orbital periods shows

\footnotetext{
* Based on proprietary and archival observations with the NASA/ESA Hubble Space Telescope, obtained at the Space Telescope Science Institute, which is operated by AURA, Inc., under NASA contract NAS $5-26555$.

† E-mail: l.e.riverasandoval@uva.nl
}

a gap between 2 to $3 \mathrm{~h}$ (see for example Knigge et al. 2011 and references therein).

The family of CVs is very heterogeneous. However, we can group them in different classes according to four key characteristics. 1) The way in which accretion occurs. Depending on the strength of the magnetic field of the WD in the $\mathrm{CV}$, the mass accretion can occur through an accretion disk (non-magnetic $\mathrm{CVs}$ ), collimated streams onto the magnetic poles (named polar CVs) or a combination of both (so called intermediate polar CVs). 2) Changes in the accretion disk. Occasional changes to a hotter and more viscous state lead to so called dwarf nova (DN) eruptions. 3) The 
ocassional presence of thermonuclear burning on the WD surface, also known as nova eruptions. 4) The presence or lack of hydrogen in the donor.

Related to the last point are the AM Canum Venaticorum (AM CVn) stars, which are CVs that have no, or very little $\mathrm{H}$ left, so Balmer lines are not present in their optical spectra (though see Israel et al. 2002, for a possible exception). These binaries have orbital periods between 5-65 mins (Carter et al. 2013). These short periods indicate that the companion is degenerate (or at least semi degenerate, which causes the period to be shorter than $\sim 75 \mathrm{~min}$, Nelson et al. 1986). It is believed that these systems harbor a WD that is accreting mass from an He rich companion, since spectra of these systems are He dominated (see Nelemans 2005, and references therein, for a review).

CVs or likely CVs have been found in different environments, such as the Galactic center, Solar neighborhood, open and globular clusters (GCs). AM CVn systems have not been identified beyond any doubt in GCs yet (though see Zurek et al. 2016). However, the study of CVs in star cluster has some advantages with respect to the study of CVs in other environments. Among those advantages are that GCs provide samples of these binaries at known distance, metallicity and age. Furthermore, the study of CVs in globulars is important, on one hand, for understanding how this population affects the evolution of the clusters (for example close binaries can help to prevent cluster core-collapse), and on the other hand, to know how the stellar interactions in a dense stellar environment, like a cluster, affect the CV evolution.

Numerical studies on the evolution of CVs in GCs have been carried out by, for example Verbunt \& Meylan (1988); Di Stefano \& Rappaport (1994); Davies (1997); Shara \& Hurley (2006); Hong et al. (2017); Belloni et al. (2017. $2017 \mathrm{~b})$. For a GC with about $10^{6}$ stars initially, Ivanova et al. (2006) found that after 10 Gyr of evolution, the number of CVs would be more than 200. This number is large compared to the number of CVs identified so far in any GC (few tens in massive clusters and only few in the less massive ones). However, because of the stellar crowdedness, intrinsic faintness and large distances (compared to the Solar neighborhood) their identification is not trivial. So far, the best way to confirm CVs is to take spectra, which has proven to be a fruitful technique (e.g. Grindlay et al. 1995 Knigge et al. 2003, 2008). Unfortunately, that is not always possible due to the faintness of these binaries and the stellar crowding in the cores of GCs (where most CVs reside), even with the use of Hubble Space Telescope (HST). Therefore, the best way to maximize the identification of possible CVs in GCs is by combining different techniques like optical variability, blue color (light from accretion is blue compared to light from normal stars), $\mathrm{H} \alpha$ excess, and X-ray emission (see for example Mukai 2017, for a recent review about the production of X-rays in $\mathrm{CVs}$ ). X-ray emission is actually a very good tracer of interacting binaries, and thanks to the good spatial resolution of Chandra it is frequently used to identify possible CVs in GCs (see below). Indeed, most of the CVs in clusters have been identified by their X-ray emission. Thus, the available sample of CVs in globulars is X-ray biased compared to the field population (most of which is optically biased, see Pretorius et al. 2013).

$\mathrm{CVs}$ are considered low luminosity X-ray sources $\left(L_{X}=\right.$ $\left.10^{29}-10^{34} \mathrm{erg} \mathrm{s}^{-1}\right)$. However, there are other accreting binaries that emit in the same range, which increases the risk of misclassification or ambiguity. Examples in 47 Tuc are the cases of the X-ray sources W42 (an accreting black hole candidate, Miller-Jones et al. 2015) and W82 (a millisecond pulsar -MSP-, Bogdanov et al.|2006) which were initially classified as CVs by Paresce et al. (1992) and Edmonds et al. (2003a, hereafter E03a), respectively. Although, we note that Edmonds et al. 2003b (from now on E03b) also suggested that W82 could be an MSP. To mitigate the risk of misclassification, the combination of photometric information at different wavelengths is important.

Clusters where multiple identifications of CVs in GCs have been made as counterparts to X-ray sources include 47 Tuc (E03a; E03b; Beccari et al. 2014), NGC 6752 (Pooley et al. 2002, Thomson et al. 2012), NGC 6397 (Grindlay et al. 2001|Cohn et al. 2010), M30 (Lugger et al.|2007), NGC 2808 (Servillat et al.|2008), M71 (Huang et al.|2010), M80 (Dieball et al. 2010), M92 (Lu et al. 2011), NGC 6388 (Maxwell et al. 2012), and $\omega$ Cen (Cool et al. 2013). These studies have shown the effectiveness of this technique and have revealed different characteristics of CVs in GCs. Nonetheless, in order to have a global idea about the properties and behavior of the CVs in clusters, a comparison among their CV populations is necessary.

Of particular interest is to know how the dynamical interactions in dense environments affect the creation and evolution of CVs. In this context, a comparison between CVs in core-collapsed and non core-collapsed clusters would help to answer that question. Core-collapse is the process that occurs when the core of the cluster has negative heat capacity, getting hotter as it loses energy to the outskirts. This means that there is a net loss of energy from the core when the dynamical interactions of stars push less massive stars in the cluster from the center to the outskirts. To counteract that effect, the stars in the core lose energy and fall deeper down the potential well, occupying a smaller volume ("collapsing"). This leads to a dramatic increase in the stellar density of the core. In terms of observations, a cluster is defined as core-collapsed if its surface brightness profile exhibits a power-law until the limit of observational resolution. On the other hand, the non core-collapsed Galactic clusters show a clear profile flattening in the central few arcseconds to arcminutes and are well fitted with a King model (King 1966).

Cohn et al. (2010) have found that in the core-collapsed cluster NGC 6397, the population of CVs is divided in two groups, with one group of CVs brighter than the other one, and more centrally concentrated. This raises the question whether CVs in non core-collapsed clusters are also divided in two groups or not, or whether the radial and brightness distribution of CVs as seen in NGC 6397 is a signature that is typical for dynamically active clusters.

47 Tuc is a well known Galactic non-core collapsed cluster that is relatively close $(\sim 4.7 \mathrm{kpc})$, shows low extinction, is massive and has a high interaction rate (Table 1). It is also the GC with the most X-ray sources identified so far (300 within the half-mass radius, $\mathrm{r}_{h}$ ), reaching a limiting luminosity in the $0.5-6 \mathrm{keV}$ band of $3 \times 10^{29} \mathrm{ergs} \mathrm{s}^{-1}$ Heinke et al. 2005, referred to from now on as H05). All these features make 47 Tuc a very good target to study close binaries, increasing the possibilities to identify many CVs. Using the 
high resolution of the Wide Field Camera 3 (WFC3) and the Advanced Camera for Surveys (ACS) on board the HST, complemented with results from Chandra, we have identified new CVs in the cluster. This includes CVs below $\sim 10^{30} \mathrm{erg}$ $\mathrm{s}^{-1}$. This is the first deep $\mathrm{H} \alpha$ study of 47 Tuc with $H S T$ that uses near-simultaneous continuum-band photometry. We designed an innovative WFC3 program to observe a large field of view of a globular cluster core to a high depth in the nearUV for the first time, using the broadest possible UV filters to increase our sensitivity. In this paper, we identify the largest number of CVs ever identified in a GC through X-ray counterpart identification, more than doubling the number of known CVs in 47 Tuc.

\section{OBSERVATIONS}

In this paper we have used the NUV data set from $H S T$ program GO 12950 taken with the UVIS channel of the WFC3 on 2013 August 13. We used a $4 \times 4$ dithering pattern that includes fractional-pixel offsets. We obtained 24 images in the filter F300X $\left(\mathrm{U}_{300}\right)$ and 8 images in the filter F390W $\left(\mathrm{B}_{390}\right)$. The total exposure times are $3881 \mathrm{~s}$ in $\mathrm{B}_{390}$ and $14256 \mathrm{~s}$ in $\mathrm{U}_{300}$. The original scale of the WFC3/UVIS images is 0. .'04 pixel $^{-1}$. We improved that resolution for the DAOPHOT and KS2 analyses (see below) by drizzling the images to obtain master frames with a pixel resolution of 0 .'02. All the objects studied here are inside the $\mathrm{r}_{h}$ of the cluster $(2.79, \mathrm{H} 05)$.

The optical images used in this work were taken with the Wide Field Channel (WFC) on the ACS under program GO 9281 in 2002 September 30, 2002 October 2/3 and 2002 October 11. We also used a dither pattern that includes fractional-pixel offsets. The optical data set consists of 10 images in the filter $\mathrm{F} 435 \mathrm{~W}\left(\mathrm{~B}_{435}\right), 20$ images in the filter $\mathrm{F} 658 \mathrm{~N}(\mathrm{H} \alpha)$, and 22 images in the filter F625W $\left(\mathrm{R}_{625}\right)$. The total exposure times are $955 \mathrm{~s}$ in $\mathrm{B}_{435}, 1320 \mathrm{~s}$ in $\mathrm{R}_{625}$, and $7440 \mathrm{~s}$ in $\mathrm{H} \alpha$. The native WFC pixel scale is 0. . 05 pixel $^{-1}$, which was improved to $0{ }^{\prime \prime} 025$ pixel $^{-1}$ after drizzling. For more details about the NUV and the optical data sets see Rivera-Sandoval et al. (2015).

In this paper we looked for CVs by identifying and classifying counterparts to Chandra X-ray sources in the cluster. We have used the catalog of X-ray sources in 47 Tuc reported by $\mathrm{H} 05$. That catalog was built using data from Chandra in 2002 with the ACIS-S instrument at the focus (281 ks exposure). These X-ray observations were scheduled to be simultaneous with the optical data used in this paper. A study of correlated X-ray and optical variability will be presented in a separate paper.

\section{DATA REDUCTION}

The image reduction and astrometric alignment of the NUV and optical images were done as described in RiveraSandoval et al. (2015). The photometric analysis of the NUV and optical images was also done as described in that paper. Additional to the DAOPHOT and KS2 analysis of the NUV images described there, we also have carried out a photometric analysis with the DOLPHOT software (Dolphin 2000 ), which worked on the individual $\mathrm{U}_{300}$ exposures. We used as a reference frame an image with extension .DRC, which is a stacked frame with the native scale of the individual images $\left(0 .{ }^{\prime \prime} 04\right.$ pixel $\left.^{-1}\right)$. It has been corrected for charge transfer efficiency degradation of the UVIS detectors, and it has also been corrected for geometric distortion. We aligned each individual image to the reference frame using the task WFC3FITDISTORT. Once the offsets in the X$\mathrm{Y}$ directions were determined, as well as the rotation with respect to the reference frame, we ran the DOLPHOT software on the $24\left(\mathrm{U}_{300}\right)+8\left(\mathrm{~B}_{390}\right)$ individual exposures. We obtained Vega-mag calibrated and aperture corrected photometric results for all the $\mathrm{U}_{300}$ and $\mathrm{B}_{390}$ images. This photometry was used for the variability analysis of the binaries described in this paper. The photometric magnitudes obtained with DOLPHOT are in general agreement with the ones obtained with DAOPHOT and KS2.

The absolute magnitudes obtained with the three software packages mentioned above were computed using a distance of $4.69 \pm 0.04 \pm 0.13 \mathrm{kpc}$ (Woodley et al. 2012). The two terms correspond to the random and the systematic errors, respectively. We also have used the reddening value towards 47 Tuc, viz. $\mathrm{E}(\mathrm{B}-\mathrm{V})=0.04 \pm 0.02$ (Salaris et al. 2007) together with the UVIS Exposure Time Calculator of HST to obtain the extinction values in the corresponding NUV filters. We obtained $\mathrm{A}\left(\mathrm{U}_{300}\right)=0.26 \pm 0.13$ and $\mathrm{A}\left(\mathrm{B}_{390}\right)=0.18$ \pm 0.09 . For the optical filters we used the conversions given in Sirianni et al. (2005) which result in $\mathrm{A}\left(\mathrm{B}_{435}\right)=0.16 \pm 0.08$, $\mathrm{A}(\mathrm{H} \alpha)=0.10 \pm 0.05$, and $\mathrm{A}\left(\mathrm{R}_{625}\right)=0.11 \pm 0.05$.

\section{RESULTS}

Out of the 300 X-ray sources found by H05 within the $\mathrm{r}_{h}$ of the cluster, 238 are in the field of view (FOV) of the NUV images and 244 are in the FOV of the optical images.

As previously mentioned, we have identified potential CVs in 47 Tuc by looking for counterparts to Chandra Xray sources in our $H S T$ NUV/optical images. We found an $\mathrm{NUV} / \mathrm{X}$-ray boresight offset of $-00^{\prime \prime} 023 \pm 0$.' 008 in right ascension and $-0.0^{\prime \prime} 003 \pm 0$. . 009 in declination in both filters (see Rivera-Sandoval et al. 2015, for more information about the NUV/X-ray alignment). We searched within $3 \sigma$ from the $\mathrm{X}$-ray positions. The size of each of these $3 \sigma$ error circles takes into account the Chandra positional error of the corresponding source (see H05), the boresight error (see above), and the HST astrometric errors (see Rivera-Sandoval et al. 2015). For each of the HST sources within the error circle, we examined their NUV and optical colors. A source is considered blue if it is located off the left side of the main sequence (MS) of the cluster in the NUV or in the $\mathrm{B}_{435}-\mathrm{R}_{625}$ versus $\mathrm{R}_{625}$ color magnitude diagrams (CMDs; Figures 1 and 2 .

Based on our photometry in the filters $\mathrm{B}_{435}, \mathrm{H} \alpha$ and $\mathrm{R}_{625}$ we have constructed a color-color diagram (CCD; Figure 3). We consider a star to have $\mathrm{H} \alpha$ excess emission if it lies above the MS in that CCD (which most of the times coincides with the source being off the left side of the MS in the $\mathrm{H} \alpha-\mathrm{R}_{625}$ versus $\left.\mathrm{R}_{625} \mathrm{CMD}\right)$.

If a star inside the $3 \sigma$ error circle has a blue color, shows an $\mathrm{H} \alpha$ excess and/or is optically variable (variability will be discussed below), then it is classified as a CV. If a star is blue but it was not detected in the $\mathrm{H} \alpha$ images and/or it does not show variability, that star is classified as a CV candidate. 
Table 1. Parameters of the globular clusters discussed in this paper that have several cataclysmic variables identified.

\begin{tabular}{|c|c|c|c|c|c|c|c|c|c|}
\hline Cluster & $\begin{array}{c}\operatorname{Mass}^{a} \\
\left(\times 10^{5} M_{\odot}\right)\end{array}$ & $\begin{array}{c}\text { Distance }^{b} \\
(\mathrm{kpc})\end{array}$ & $\begin{array}{l}\mathrm{Age}^{c} \\
(\mathrm{~Gy})\end{array}$ & $\begin{array}{l}\mathrm{r}_{h}^{d} \\
\left({ }^{\prime}\right)\end{array}$ & {$[\mathrm{Fe} / \mathrm{H}]^{c}$} & $\mathrm{E}(\mathrm{B}-\mathrm{V})^{e}$ & $\begin{array}{c}\rho_{0}^{e} \\
\left(L_{\odot} / \mathrm{pc}^{3}\right)\end{array}$ & $\begin{array}{c}\Gamma^{f} \\
(\text { total })\end{array}$ & $\begin{array}{c}\text { Core } \\
\text { collapsed? }\end{array}$ \\
\hline 47 Tuc & $6.45 \pm 0.4$ & $4.69 \pm 0.04 \pm 0.13$ & $11.75 \pm 0.25$ & 2.79 & -0.76 & 0.04 & 4.88 & 1000 & $\mathrm{No}$ \\
\hline NGC 6397 & $1.1 \pm 0.1$ & $2.2_{-0.7}^{+0.5}$ & $13.00 \pm 0.25$ & 2.33 & -1.99 & 0.18 & 5.76 & 84.1 & Yes \\
\hline NGC 6752 & $2.82 \pm 0.4$ & $4.0 \pm 0.2$ & $12.50 \pm 0.25$ & 1.9 & -1.55 & 0.04 & 5.04 & 401 & Yes \\
\hline
\end{tabular}

${ }^{a}$ Taken from Kimmig et al. (2015) for 47 Tuc and from Heyl et al. (2012) for NGC 6397.

${ }^{b}$ Taken from Woodley et al. (2012) for 47 Tuc, Heyl et al. (2012) for NGC 6397, and Harris (1996, 2010 revision) for NGC 6752.

$c$ Taken from VandenBerg et al. (2013).

${ }^{d}$ Taken from H05 for 47 Tuc, Cohn et al. (2010) for NGC 6397 and from Lugger et al. (2017) for NGC 6752.

$e$ Taken from Harris (1996, 2010 revision).

$f$ Taken from Bahramian et al. (2013).

$g$ Taken from Trager et al. (1995).

We have applied the same criteria to the previously suggested CVs by other authors (see for example Knigge et al. 2002. E03a, H05, Beccari et al. 2014). In that case if the star shows $\mathrm{H} \alpha$ excess and/or is variable in our images, then it is still considered as a CV. On the other hand, if the star is blue and visible in the $\mathrm{H} \alpha$ images, but it does not show an $\mathrm{H} \alpha$ excess and/or variability, then an alternative explanation for this behavior is considered.

Using our CCD, we estimated the $\mathrm{H} \alpha$ equivalent width (EW) of our CVs and CV candidates by comparing their dereddened $\mathrm{H} \alpha-\mathrm{R}_{625}$ color to the expected $\mathrm{H} \alpha-\mathrm{R}_{625}$ color for an MS star of the same $\mathrm{B}_{435}-\mathrm{R}_{625}$ color. The latter were computed using the Synphot packag $£^{1}$ We observe that all the CVs and CV candidates have $\mathrm{EW}(\mathrm{H} \alpha) \lesssim-5 \AA$, which are consistent with EWs found for CVs in the field (van den Berg et al. 2009, Szkody et al. 2011).

To complement our analysis, we have also studied variability of the objects discussed here as CVs and CV candidates. This is an important tool to identify CVs, since variability is expected from these binaries due to flickering, eclipses or ellipsoidal variability. We have used the root main square (rms) deviation about the $\mathrm{U}_{300}$ median magnitude obtained with DOLPHOT as a measure of variability. To reduce the possible scatter due to photometric issues in the 24 measurements for each object, we have only taken into account photometric measurements labeled by DOLPHOT as good quality measurements (flag equal zero). The possible influence of outlier ${ }^{2}$ has been reduced by using an iterative $3 \sigma$ clipping algorithm (which removes most of the measurements affected by noise, cosmic rays, hot pixels, etc.). To quantify the level of variability for each $\mathrm{CV}$ and CV candidate, we have used the percentile technique. Thus we have drawn lines along the well defined sequence of non-variables (or very-small-amplitude variables) in this diagram. They represent the levels above which $95 \%$ and $99 \%$ of the stars at a given magnitude are found. If an optical source is on or above the $95 \%$ confidence level line, then we consider it variable.

It is possible that periodic behavior is not observed (even when present) for many objects because of the short

\footnotetext{
1 www.stsci.edu/institute/software_hardware/stsdas/synphot 2 Measurements that result in magnitudes smaller or larger than $m \pm 3 \sigma$, where $m$ is the median magnitude and $\sigma$ is the standard deviation.
}

time coverage (few hours) of our observations. However, we detected an eclipse of the known CV AKO 9 Auriere et al. 1989 see Section 4.1). In order to investigate the presence of outbursts or eclipses we have also constructed light curves in the filter $\mathrm{U}_{300}$. We thus detected four $\mathrm{CVs}$ that exhibit large-amplitude variability at the time of our observations (see Section 4.2.

Using the methods mentioned above, we classified $43 \mathrm{X}$ ray sources in the cluster as CVs and CV candidates (with likely non degenerate donors). Of all those, 22 are new identifications. All of these CVs and CV candidates are X-ray sources with luminosities $L_{X(0.5-6 \mathrm{keV})} \sim 5 \times 10^{29}-2 \times 10^{32}$ $\mathrm{erg} \mathrm{s}^{-1}$ (H05). In the NUV CMD, most of these CVs and CV candidates lie on or near the primary WD cooling sequence of the cluster, while few others fall on the Small Magellanic Cloud ${ }^{3}$ (SMC) MS. For the objects that lie on the SMC MS, we think that it is unlikely that they actually belong to that galaxy because only a small fraction of SMC stars fall inside our field; the chance of finding superpositions with X-ray sources with luminosities of the order $10^{32}-10^{34} \mathrm{erg} \mathrm{s}^{-1}$ (at the distance of the SMC, $\sim 60 \mathrm{kpc}$ ), is pretty low. Also, these X-ray luminosities would be very high for being isolated MS or active binary (AB; $L_{X}<10^{31} \mathrm{erg} \mathrm{s}^{-1}$ ) stars (for instance, W51 would have an X-ray luminosity of $\sim 10^{34} \mathrm{erg}$ $\mathrm{s}^{-1}$ ). We note that the primary source of spurious identifications to our sample would be single WDs that happen to land in our error circles. These WDs would lie on the WD sequence, but will not show $\mathrm{H} \alpha$ excess or variability.

The apparent magnitudes of the $43 \mathrm{CVs}$ and CV candidates, together with their astrometric results are given in Table 5 . Finding charts for some of them are shown in Appendix B In Section 4.3 we discuss two objects with optical (specifically $\mathrm{H} \alpha$ ) properties that distinguish them from the CVs and CV candidates discussed in Section 4.1. viz. a candidate AM CVn system and another intriguing, but unclassified object. in Section 4.4 we present four candidate counterparts to Chandra sources that show striking similarities to known MSP optical counterparts; the difference is that no radio source has been detected at the position of these four sources, yet. Finally in Section 4.5 we discuss in

\footnotetext{
3 The outskirts of the SMC are behind 47 Tuc, leading to some contamination of the 47 Tuc CMD by SMC stars around $\mathrm{U}_{300^{-}}$ $\mathrm{B}_{390} \sim 0.1$ and $\mathrm{M}_{B 390} \gtrsim 8.5$ in Figure 1 and $\mathrm{B}_{435}-\mathrm{R}_{625} \sim 0.5$ and $\mathrm{M}_{R 625} \gtrsim 8$ in Figure 2
} 


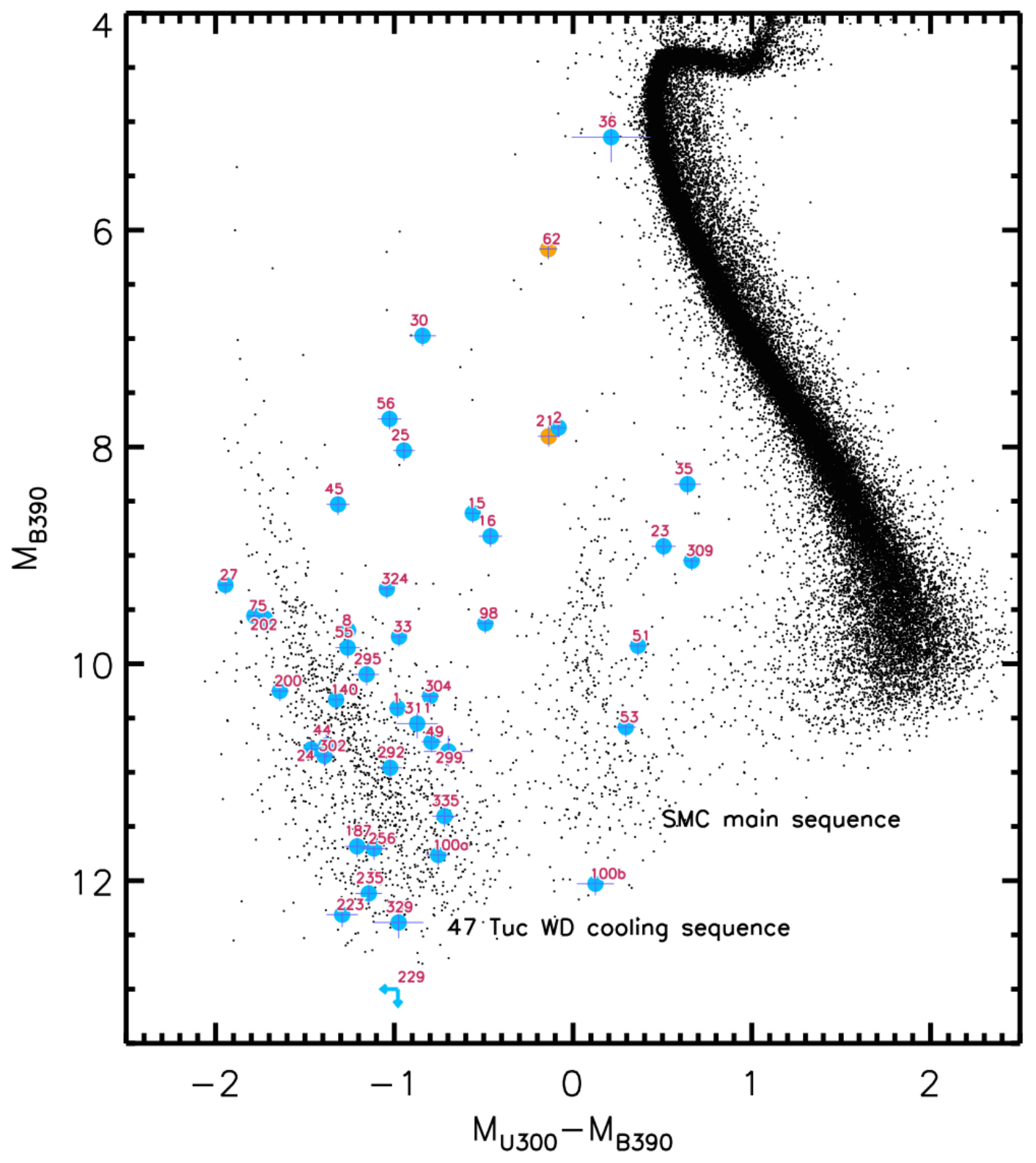

Figure 1. $\mathrm{U}_{300}-\mathrm{B}_{390}$ versus $\mathrm{B}_{390} \mathrm{CMD}$ based on DAOPHOT photometry of our NUV images. Each CV and CV candidate is represented with a blue circle and its respective $\mathrm{W}$ number. The AM CVn candidate $\mathrm{W} 62_{U V}$ and the unclassified object $\mathrm{W} 21_{U V}$ are marked with an orange circle. Apparent magnitudes were converted to absolute magnitudes using a distance to 47 Tuc of $4.69 \mathrm{kpc}$ and reddening $\mathrm{E}(\mathrm{B}-\mathrm{V})=0.04$. Mean magnitudes are given for the variable objects $\mathrm{W} 2_{U V}, \mathrm{~W} 15_{U V}, \mathrm{~W} 23_{U V}$ and $\mathrm{W} 27_{U V}$. The $\mathrm{CV}$ candidate $\mathrm{W}_{22} 9_{U V}$ was just detected in the $\mathrm{U}_{300}$ band and thus is plotted in this CMD using an upper limit in $\mathrm{B}_{390}$.

detail the probability for chance coincidences between Chan$d r a$ sources and optical sources with CV-like properties.

From now on we use the subscript "UV" to refer to the NUV counterpart to the corresponding "W" X-ray source from the catalog of $\mathrm{H} 05$, e.g. $\mathrm{W} 1_{U V}$ as the counterpart of W1. We also use the abbreviation "NUV CMD" for the $\mathrm{U}_{300}$ $\mathrm{B}_{390}$ versus $\mathrm{B}_{390} \mathrm{CMD}$, "optical CMD" for the $\mathrm{B}_{435}-\mathrm{R}_{625}$ versus $\mathrm{R}_{625} \mathrm{CMD}$, and "H $\alpha$ CMD" for the $\mathrm{H} \alpha-\mathrm{R}_{625}$ versus $\mathrm{R}_{625} \mathrm{CMD}$.

\subsection{Cataclysmic variables in 47 Tuc}

\subsubsection{Previously identified $C V s$ and $C V$ candidates}

The nineteen CVs and CV candidates associated with the Xray sources W1, W2, W8, W15, W21 (see Section 4.3), W25, W27 (V3), W30, W33, W35, W36 (AKO 9), W44, W45, W49, W51, W53, W56, W120 and W122 were previously suggested by Paresce et al. (1992), Paresce \& de Marchi (1994), Shara et al. (1996), Grindlay et al. (2001) and E03a to be CVs. Where clearly detected in our data, these CVs are 

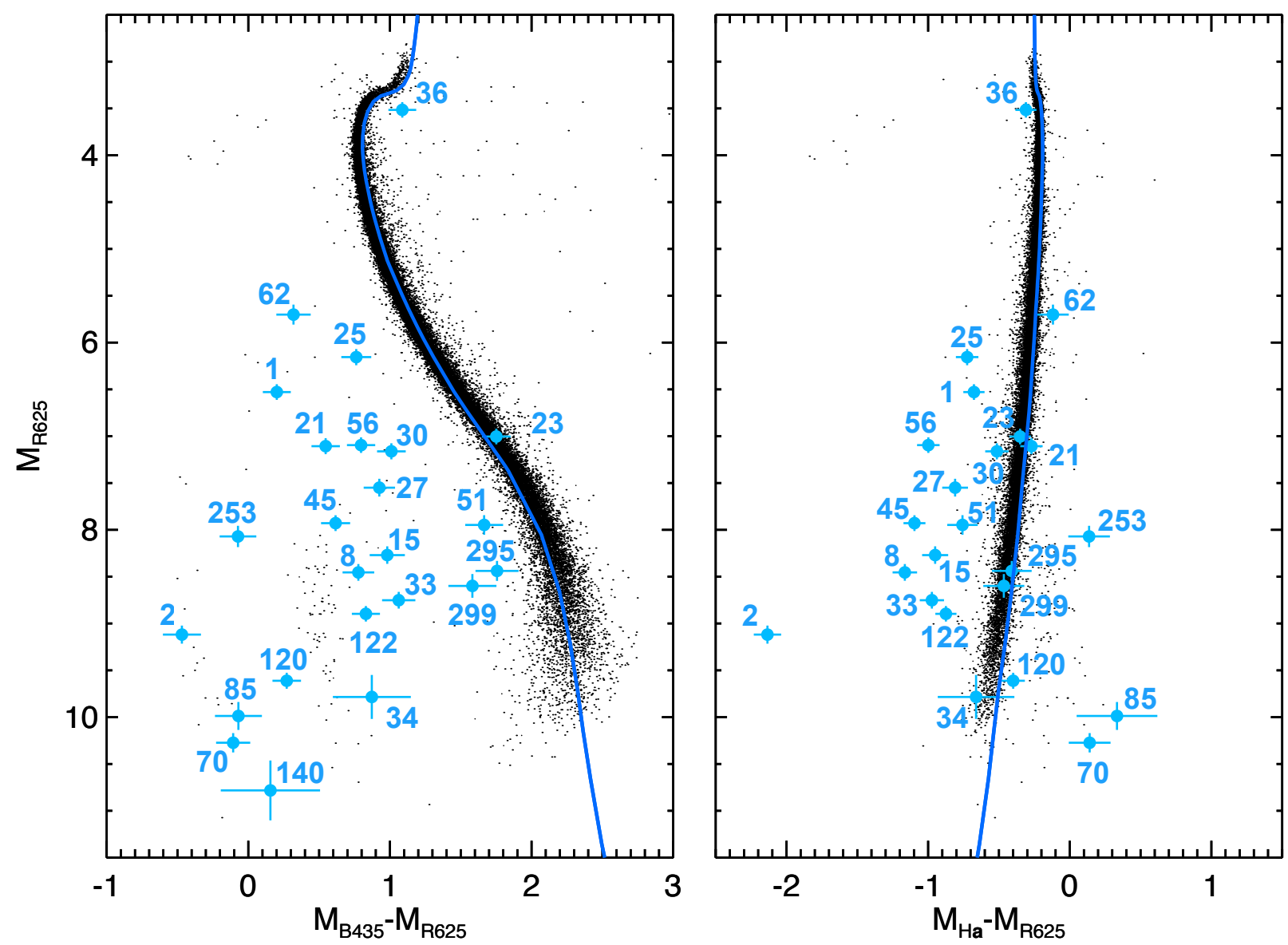

Figure 2. $B_{435}-R_{625}$ versus $R_{625}$ (left) and $H \alpha-R_{625}$ versus $R_{625}$ (right) CMDs. Counterparts discussed in this paper are plotted with blue circles. Calibrated Vega-mag magnitudes were converted to dereddened absolute magnitudes using a distance to 47 Tuc of $4.69 \mathrm{kpc}$ and reddening $\mathrm{E}(\mathrm{B}-\mathrm{V})=0.04$. The mean magnitude is given for $\mathrm{W} 2_{U V}$. Overplotted are isochrones for an age of $11 \mathrm{Gyr}$ and $\mathrm{Z}=0.004$ for the corresponding HST filters (Bressan et al. 2012).

blue in our NUV and optical CMDs, and have an $\mathrm{H} \alpha$ excess (Figures 1 and 2). Exceptions are $\mathrm{W} 120_{U V}$ and $\mathrm{W} 122_{U V}$ which are not in the FOV of our NUV images, W35 $U V$ that is surrounded by bright stars preventing $\mathrm{R}_{625}$ and $\mathrm{H} \alpha$ photometry, as well as W53 ${ }_{U V}$ and $\mathrm{W} 44_{U V}$ in which diffraction spikes affect the optical photometry. The object $\mathrm{W} 49_{U V}$ was detected neither in $\mathrm{H} \alpha$ nor $\mathrm{R}_{625}$. Two additional sources with WD-like colors were found within the $2 \sigma$ error circle of W49. For one of them optical photometry was derived but due to contamination by a nearby saturated star the photometry is not reliable. The other WD-like object was not detected in the optical bands. Among the three possible candidates, only one $\left(\mathrm{W} 49_{U V}\right)$ shows variability (Figure 4 ).

We also note that $\mathrm{W} 120_{U V}$ lies to the right of the $\mathrm{H} \alpha-$ $\mathrm{R}_{625}$ MS as some faint CVs in the cluster NGC 6397 (Cohn et al. 2010) do. However, considering its blue color (Figure 2 left), the CCD (Figure 3 shows an $\mathrm{H} \alpha$ excess that corresponds to a line with $\mathrm{EW}(\mathrm{H} \alpha)$ of $-30 \AA$, which is consistent with the $\mathrm{EW}(\mathrm{H} \alpha)$ of typical CVs.

The objects $\mathrm{W} 1_{U V}, \mathrm{~W} 8_{U V}, \mathrm{~W} 27_{U V}, \mathrm{~W} 33_{U V}, \mathrm{~W} 44_{U V}$, $\mathrm{W} 45_{U V}$ and $\mathrm{W} 49_{U V}$ lie on the WD cooling sequence of the NUV CMD, which suggests a large contribution from the WD and/or accretion disk to the NUV spectrum. On the other hand, the colors of the CVs W15UV ${ }_{U}$ W $25_{U V}$, W30 $0_{U V}$, $\mathrm{W} 355_{U V}, \mathrm{~W} 36_{U V}, \mathrm{~W} 51_{U V}, \mathrm{~W} 53_{U V}$ and $\mathrm{W} 56_{U V}$ suggest a larger contribution from the companion star, though it is also possible that some of them (like $\mathrm{W} 25_{U V}$ and $\mathrm{W} 56_{U V}$ ) have emission dominated by hot, relatively low-mass WDs. In the case of $\mathrm{W} 36_{U V}$ (AKO 9), which harbors a subgiant secondary star, the NUV emission is dominated by the (edge-on) accretion disk (Knigge et al. 2003), and it is the brightest NUV object among the CV and CV candidates discussed in this paper.

Among the stars that show variability at a confidence level equal or higher than $95 \%$ in $\mathrm{U}_{300}$ (Figure 4) are $\mathrm{W} 2_{U V}, \mathrm{~W} 8_{U V}, \mathrm{~W} 15_{U V}, \mathrm{~W} 25_{U V}, \mathrm{~W} 27_{U V}, \mathrm{~W} 30_{U V}, \mathrm{~W} 33_{U V}$, $\mathrm{W} 35_{U V}, \mathrm{~W} 36_{U V}, \mathrm{~W} 45_{U V}, \mathrm{~W} 49_{U V}, \mathrm{~W} 51_{U V}$ and $\mathrm{W} 56_{U V}$. Given that the expected number of variable blue objects or variable WDs associated by chance within these error circles is small (Section 4.5), it is likely that they are the real counterparts to the respective X-ray sources. The objects $\mathrm{W} 1_{U V}$, $\mathrm{W} 44_{U V}$ and $\mathrm{W} 53_{U V}$, do not show variability in $\mathrm{U}_{300}$. However, E03b (see for instance their Figure 9) found that they display long term variability in the filters $\mathrm{F} 814 \mathrm{~W}$ and/or F300W, which also suggests that they are the correct X-ray counterparts. The objects $\mathrm{W} 120_{U V}$ and $\mathrm{W} 122_{U V}$ are out- 


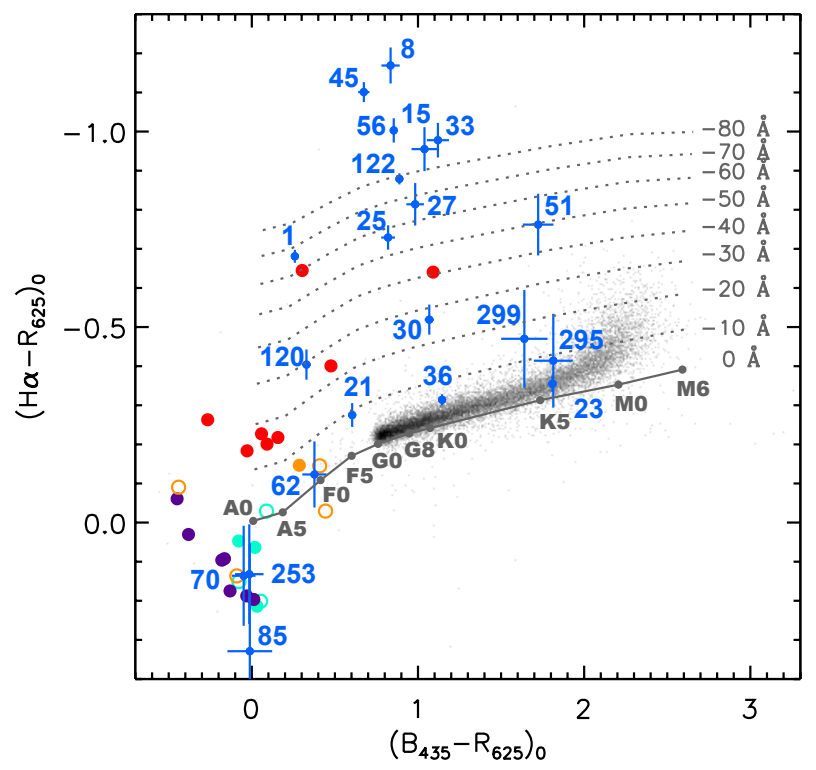

Figure 3. Color-color diagram of 47 Tuc that shows the optical counterparts to Chandra X-ray sources in the cluster discussed in this paper (small blue circles with error bars). The photometry has been dereddened using $\mathrm{E}(\mathrm{B}-\mathrm{V})=0.04$. Synthetic colors of various spectral types were computed with the Synphot package using low-metallicity ( $\log \mathrm{Z}=-2.5$ ) Castelli-Kurucz model spectra, and are shown as dark gray circles. The solid gray line corresponds to $\mathrm{EW}(\mathrm{H})=0 \AA$ and the dotted lines show increments of $\Delta \mathrm{EW}(\mathrm{H} \alpha)=-10 \AA$, which result from adding a gaussian emission line with FWHM of $25 \AA$. Orange, cyan and red circles are colors obtained from AM CVn, DA WD and CV spectra from the Sloan Digital Sky Survey (SDSS) by Carter et al. (2013), respectively. The purple circles are colors obtained for DA WDs model spectra with Synphot. For some SDSS systems a small fraction of the $\mathrm{B}_{435}$ filter bandpass is not covered by the spectra, which means that the actual $\mathrm{B}_{435}-\mathrm{R}_{625}$ colors of the SDSS binaries are slightly bluer than plotted in this figure (open circles). Filled circles correspond to good $\mathrm{B}_{435}$ coverage. Other stars in the cluster are denoted with black dots.

side of the NUV FOV. But they are also clearly variable in other bands (E03b), supporting their classification as CVs.

For AKO 9 the variability is related to eclipses (a partial light curve of this 1.1 day accreting binary is shown in Figure 5). For the other CVs the nature of variability appears more stochastic and could be due to flickering. The variability of $\mathrm{W} 2{ }_{U V}, \mathrm{~W} 15_{U V}$ and $\mathrm{W} 27_{U V}$ is discussed in more detail in Section 4.2

\subsubsection{Confirmation of previously suggested $C V s$}

The stars $\mathrm{W} 16_{U V}, \mathrm{~W} 55_{U V}$ and $\mathrm{W} 140_{U V}$ were previously mentioned as possible CVs (E03a). These objects were not firmly identified as CVs due to the stellar crowdedness in the optical images of these authors. Our results support the classification of these objects as CVs. These three objects have no $\mathrm{H} \alpha$ information (there are saturated stars nearby or very close neighbors) but they were clearly identified in the NUV images. In the NUV CMD, W16 $6_{U V}$ lies between the primary WD cooling sequence and the MS of the SMC, while $\mathrm{W} 55_{U V}$ and $\mathrm{W} 140_{U V}$ lie on, or near the primary WD cool- ing sequence. The objects $\mathrm{W} 16_{U V}$ and $\mathrm{W} 140_{U V}$ display variability at a confidence level higher than $99 \%$, which strongly supports their classification as CVs. Variability for W55 was not detected.

\subsubsection{Unidentified previously suggested CVs}

We note that for the X-ray sources W10 and W20, previously cataloged as CV candidates based on the presence of saturated/bright stars and X-ray properties (E03a, E03b, $\mathrm{H} 05$ ), we did not find signals of any blue object within the $3 \sigma$ error circle in the NUV images that could indicate the presence of an accreting WD. We obtained the same results from the analysis at optical wavelengths. Both W10 and W20 have relatively bright (giant, subgiant or MS) stars in the X-ray error circles, which may prevent the identification of true, faint counterparts (E03a), such as CVs or MSPs. Alternatively, the bright stars may be the true counterparts, in which case they are likely RS CVn-type coronally active binaries. Apart from W10, W20, W32 and W71 (the latter two reclassified as likely ABs), all the other CVs and CV candidates mentioned by E03a, E03b and H05 are presented in our paper.

\subsubsection{New $C V$ s and $C V$ candidates}

In this work we have classified $22 \mathrm{X}$-ray sources as new $\mathrm{CVs}$ and $\mathrm{CV}$ candidates (most of which were previously unclassified X-ray sources). The respective counterparts are $\mathrm{W} 23_{U V}, \mathrm{~W} 24_{U V}, \mathrm{~W} 75_{U V}, \mathrm{~W} 98_{U V}, \mathrm{~W} 100_{U V-\mathrm{a} \text { or }-\mathrm{b}}$, $\mathrm{W} 187_{U V}, \mathrm{~W} 200_{U V}, \mathrm{~W} 202_{U V}, \mathrm{~W} 223_{U V}, \mathrm{~W} 229_{U V}, \mathrm{~W} 235_{U V}$, $\mathrm{W} 256_{U V}, \mathrm{~W} 292_{U V}, \mathrm{~W} 295_{U V} \mathrm{~W} 299_{U V}, \mathrm{~W} 302_{U V}, \mathrm{~W} 304_{U V}$, $\mathrm{W} 309_{U V}, \mathrm{~W} 311_{U V}, \mathrm{~W} 324_{U V}, \mathrm{~W} 329_{U V}$, and $\mathrm{W} 335_{U V}$. All of them show blue colors in the NUV except W229 ${ }_{U V}$, which was only detected in the $\mathrm{U}_{300}$ stacked image, and therefore we were not able to investigate its variability in that band. The non-detection of $\mathrm{W}_{22} 29_{U V}$ in the $\mathrm{B}_{390}$ filter indicates it is a blue object. Most of the counterparts to these 22 X-ray sources lie on the WD cooling sequence of the NUV CMD, indicating the strong contribution from the accreting WD to the NUV light. Because of intrinsic faintness, stellar crowding, bright saturated stars nearby and diffraction spikes, it was not possible to get optical photometry, including $\mathrm{H} \alpha$, for all of the 23 optical stars (counting $\mathrm{W} 100_{U V-a}$ and $\mathrm{W} 100_{U V-b}$ separately). In the filter $\mathrm{B}_{435}$ photometry was derived for fourteen objects. $\mathrm{H} \alpha$ information was obtained for three stars $\left(\mathrm{W} 23_{U V}, \mathrm{~W} 295_{U V}\right.$ and W299 $U V$ ), which supports their identification as CVs. They show $\mathrm{EW}(\mathrm{H} \alpha) \lesssim-5 \AA$ (up to $-30 \AA$ for $\mathrm{W} 299_{U V}$ ), also their different location in the optical and NUV CMDs with respect to the MS or WD sequence show that they are not single-temperature WDs, and thus most likely contain WDs and donor stars. Variability at the confidence level of $95 \%$ or higher was detected for nine out of the 23 stars $\left(\mathrm{W} 23_{U V}, \mathrm{~W} 200_{U V}, \mathrm{~W} 256_{U V}, \mathrm{~W} 292_{U V}\right.$, $\mathrm{W} 295_{U V} \mathrm{~W} 299_{U V}, \mathrm{~W} 304_{U V}, \mathrm{~W} 309_{U V}$ and $\left.\mathrm{W} 311_{U V}\right)$. Because of that, they have only a small chance of being random coincidences compared to the blue stars found in the $3 \sigma$ error circles of the other thirteen sources that are newly classified as candidate CVs. Eclipses or ellipsoidal variations were not found in any of the new 23 (counting W100 $U V-a$ and $\mathrm{W} 100_{U V-b}$ separately) systems. For the non variable 
counterparts, it is possible that they are variable in other bands different than $\mathrm{U}_{300}$, like the previously discussed cases of $\mathrm{W} 1_{U V}, \mathrm{~W} 44_{U V}$ and $\mathrm{W} 53_{U V}$. Another possibility is that those systems have long term variations that were missed because of our short time coverage. It is also possible that due to the $\sim 1.5 \mathrm{hr}$ observational gap (between both visits due to Earth occultation), orbital variations, like brief eclipses, were missed. We note that unlike the rest of CVs and $\mathrm{CV}$ candidates in the cluster, which display a blue color in the optical CMD, the object $\mathrm{W} 23_{U V}$ lies on the MS of the optical CMD. Using the isochrones computed by Bressan et al. (2012) we determined that W23 ${ }_{U V}$ could have an optical companion with mass $0.55-0.6 M_{\odot}$. This object is also highly variable in the NUV and thus will be further discussed in Section 4.2

The X-ray sources W23, W24, W75, W100, W200, W202, W256, W299, W302, W304 and W335 are also among the X-ray sources for which we found multiple potential counterparts, and more information about them is given below (Section 4.1.5).

\subsubsection{Confused X-ray sources and X-ray sources with multiple potential counterparts}

The high stellar crowding in 47 Tuc can lead to multiple potential NUV/optical counterparts within a single Chandra error circle. This may be due to chance coincidence of one or more non-X-ray-emitting stars in the error circle, and/or due to X-ray sources lying too close to each other to be resolved by Chandra. H05 suggest that of order $14 \%$ of Xray sources in the core brighter than $\sim 10^{30} \mathrm{erg} / \mathrm{s}$ are not detected, due to being "confused" with similar or brighter $\mathrm{X}$-ray sources. This is consistent with the evidence that 3 of 22 MSPs (47 Tuc F, 47 Tuc G, and 47 Tuc O) are confused with other sources (H05), two of which are other MSPs. Such "confused" X-ray sources are also more difficult to analyze in the NUV and optical. This is because they have larger error circles. However, most of the NUV/optical sources in the error circles have colors that are compatible with those expected from MS stars, which suggests that they are aligned by chance with the X-ray source.

For twelve X-ray sources we found more than one interesting NUV or optical source inside the X-ray error circle, of which at least one is a CV or CV candidate. These are W23, W24, W35, W75, W100, W200, W202, W256, W299, W302, W304 and W335. Six of these exhibit NUV variability. The sources W24, W35, W75, W202 and W302 are among the list of X-ray "confused" sources in H05 and are therefore likely source blends. For statistical purposes we have just given upper limits for the X-ray luminosities of these CV candidates (except for W100; Table 5), since an accurate and reliable splitting of the luminosities among the number and kind of X-ray sources identified for each of these $\mathrm{W}$ sources is impossible given the available information. Results about the other possible counterparts (that are not CVs) for these $\mathrm{X}$-ray sources will be shown in a separate paper. We stress that in this work we focus on the results about CVs and CV candidates.

From our NUV analysis we found that all the X-ray sources mentioned above have at least one blue object within the $3 \sigma$ error circle. An exception is W75, for which the WDlike object identified lies outside $3 \sigma$. A counterpart at $2.5 \sigma$ has been proposed by E03a, but other counterparts outside $3 \sigma$ have also been associated with that X-ray source (e.g. Knigge et al. 2002, Knigge et al. 2008).

The sources W23, W24, W256, W299 and W304 were previously classified as ABs (Albrow et al. 2001, E03b, H05). For all these sources we identified the corresponding $\mathrm{ABs}$ based on published finding charts, or on the coordinates of the associated optical variables. However, for the first time we also identified a blue object inside the $3 \sigma$ error circles (all but W23 ${ }_{U V}$ lying on the WD sequence in the NUV CMD), which in the cases of W23, W24 and W304 are closer than the ABs to the center of the error circle. On the other hand W200, W202, W302 and W335, were until now unclassified X-ray sources. For all of them (including W35) we found two possible counterparts, a blue object and an AB. An exception is W200, which apart from the presumable CV (at $2 \sigma$ from the center of the X-ray circle) there is a blue straggler star (at $0.43^{\prime \prime}$ or $4 \sigma$ from the center of the X-ray error) that could contribute to the X-ray flux.

The source W100 is a peculiar case for which we found two blue objects within the $3 \sigma$ error circle (see Appendix B). They lie at $0.25^{\prime \prime}$ or $1.5 \sigma\left(\mathrm{W} 100_{U V-a}\right)$ and $0.18^{\prime \prime}$ or $1 \sigma$ $\left(\mathrm{W} 100_{U V-b}\right)$ from the center of the circle. Only $\mathrm{W} 100_{U V-a}$ was detected in the filter $\mathrm{B}_{435}$. Based on the available information it is impossible to tell which one is the correct counterpart to W100, or whether W100 is a blended X-ray source with two CV counterparts (though this would be less likely).

\subsection{CVs with large-amplitude NUV variability}

In this section, we present results for the counterparts to the X-ray sources W2, W15, W23 and W27, which display largeamplitude NUV variability during the time of our NUV observations. In Figure 6 we show their DOLPHOT light curves; these are in good agreement with those obtained with KS2. Information about the NUV and optical colors of these four objects were discussed in Sections 4.1.1 (W2, $\mathrm{W} 15, \mathrm{~W} 27$ ) and 4.1.4 (W23). Based on an exquisite $H S T$ WFPC2 data set of more than $600 \mathrm{~V}$ and $I$ exposures obtained over an $8.3 \mathrm{~d}$ period, E03b performed a variability analysis of many optical counterparts to Chandra sources in 47 Tuc, the results of which we compare with more recent results and our findings on a case-by-case basis below.

For the CV $\mathrm{W} 2_{U V}$, no indication of periodicity was found when folding the $\mathrm{U}_{300}$ light curve on any of the possible optical periods $(2.2,5.9$ and $8.2 \mathrm{hr})$ or X-ray period $(6.287 \mathrm{hr})$ suggested by E03b. However, recently Israel et al. (2016) used a larger Chandra data set to do a period search and found that this system shows eclipses with a period of $2.4025 \mathrm{~h}$. Using that period, we did not find a periodic pattern in our NUV light curves; the optical data on the other hand, do show periodic behavior and deep, eclipselike features, most notable in the $\mathrm{R}_{625}$ filter (see Figure 7 for the folded $R_{625}$ and $U_{300}$ light curves). Interestingly, the amplitude of the variations observed by E03b in their light curves from 1999 is small (about $0.5 \mathrm{mag}$ in $V$ ) compared to our findings. W2 is also matched to the miscellaneous blue variable WF2-V48 in Albrow et al. (2001), who already noted that this star could be a CV. Their $V$ light curve, which may be affected by a nearby diffraction spike, shows deep sharp dips, resembling the behavior of our $R_{625}$ light 


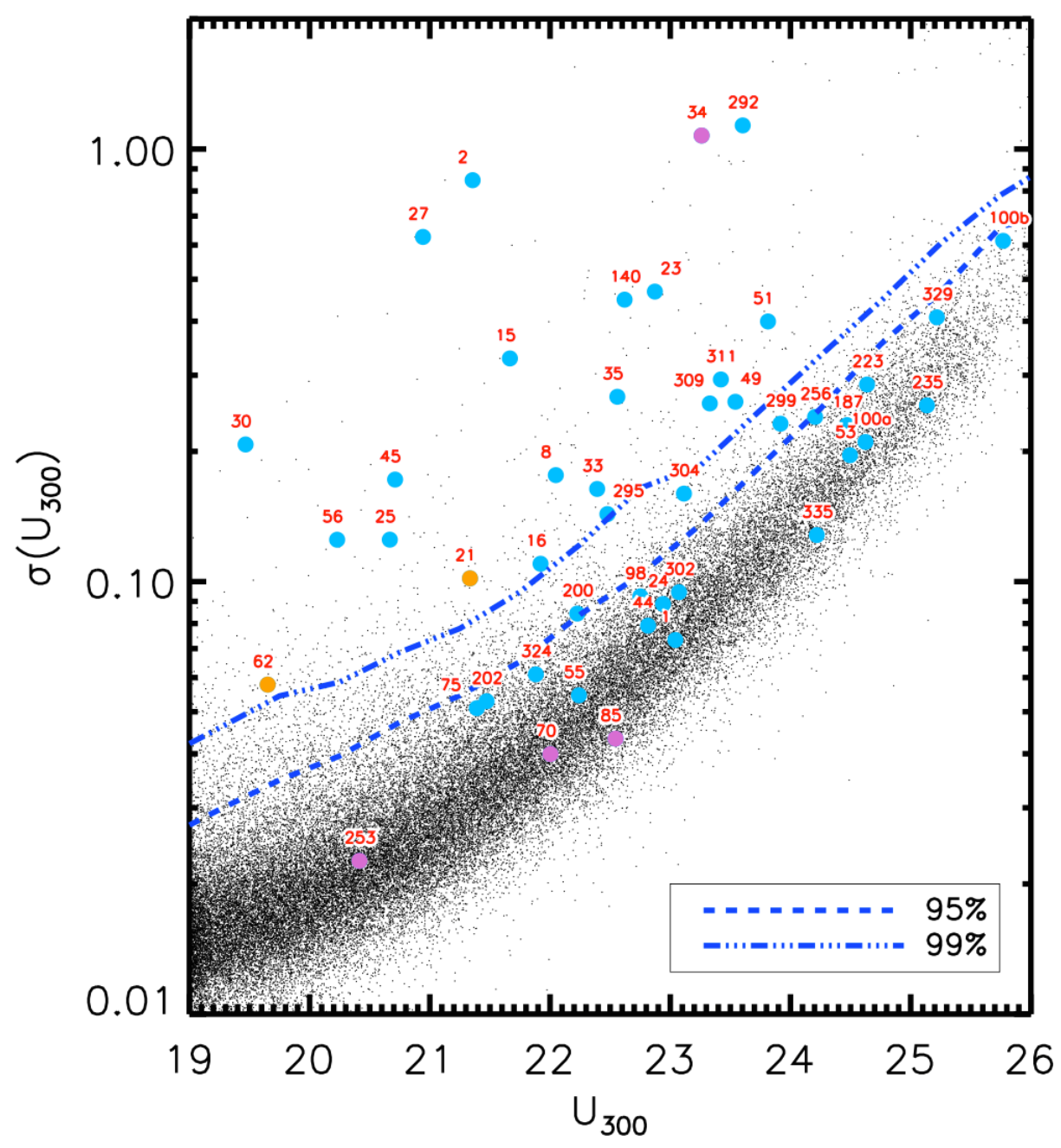

Figure 4. Variability diagram in the filter $\mathrm{U}_{300}$, showing the rms variability in the (at most 24) $\mathrm{U}_{300}$ magnitudes extracted by DOLPHOT from the individual exposures, versus the average magnitude. Outliers were filtered by using $3 \sigma$ clipping algorithm. The CVs and CV candidates are represented with blue circles. The blue dashed lines represent the $95 \%$ and $99 \%$ confidence level of variability, as computed by the percentiles in the $\sigma\left(\mathrm{U}_{300}\right)$ distribution below which $95 \%$ and $99 \%$ of the stars are found (percentiles computed in bin widths of $0.5 \mathrm{mag}$ ). Other stars in the cluster are represented with black dots. The AM CVn candidate W62 $U V$ and the unclassified object W21 $1_{U V}$ show variability at a confidence level higher than $99 \%$ and are marked with orange circles. The candidates to MSP companions are market with pink circles. The eclipsing CV AKO 9 is not shown because its brightness makes it fall outside the frame. W120 $U V$ and W122 $U V$ are outside of the NUV FOV, thus there are no NUV results for them. The CV candidate W229 $9_{U V}$ was just detected in the $\mathrm{U}_{300}$ stacked image, and so it is not included in this diagram.

curve. Eclipses that come and go on a time scale of years could be due to long-term changes in the system's configuration with respect to the observer, for example due to precession of the accretion disk, or changes in the thickness of the disk. Despite the absence of a periodic NUV signal, the $\mathrm{U}_{300}$ light curve shows magnitude variations of $\sim 3 \mathrm{mag}$ (Figure 6) within the time span of our observations $(\sim 8$ hr). These variations cannot be attributed to contamination by neighboring stars, since $\mathrm{W} 2_{U V}$ is the only star inside the $3 \sigma$ error circle. Non-periodic, large-amplitude variability has been observed for highly magnetic CVs, and is associated with changes between high and low accretion states. State switches can occur on a time scale of about one or two weeks (see for example HS 0943+1404; Rodríguez-Gil et al. 2005) but large amplitude variations in time-scales of tens of min- utes have also been observed for magnetic CVs (like in AE Aqr e.g. Zamanov et al. 2012); if W2 is an intermediate polar (the presence of a disk is required to account for the observed/unobserved eclipses at different epochs), we could have caught the system during a high/low state transition.

W15 $U V$ is an eclipsing CV with an optical period of $4.238 \mathrm{hr}$ (E03b). In the $\mathrm{U}_{300}$ we did not find the eclipse for this object when folding the light curve using that period. However, we observe photometric variations of $\sim 2.5$ mag in the same band (see Figures 6 and 8 ). This contrasts with the optical light curve presented by E03b which shows ellipsoidal variations, and does not show a large decay or increase on a time scale of hours like we observe. Visual inspection of the individual images suggests that the variations obtained in the NUV are authentic. Like is the case of $\mathrm{W} 2_{U V}$, this 


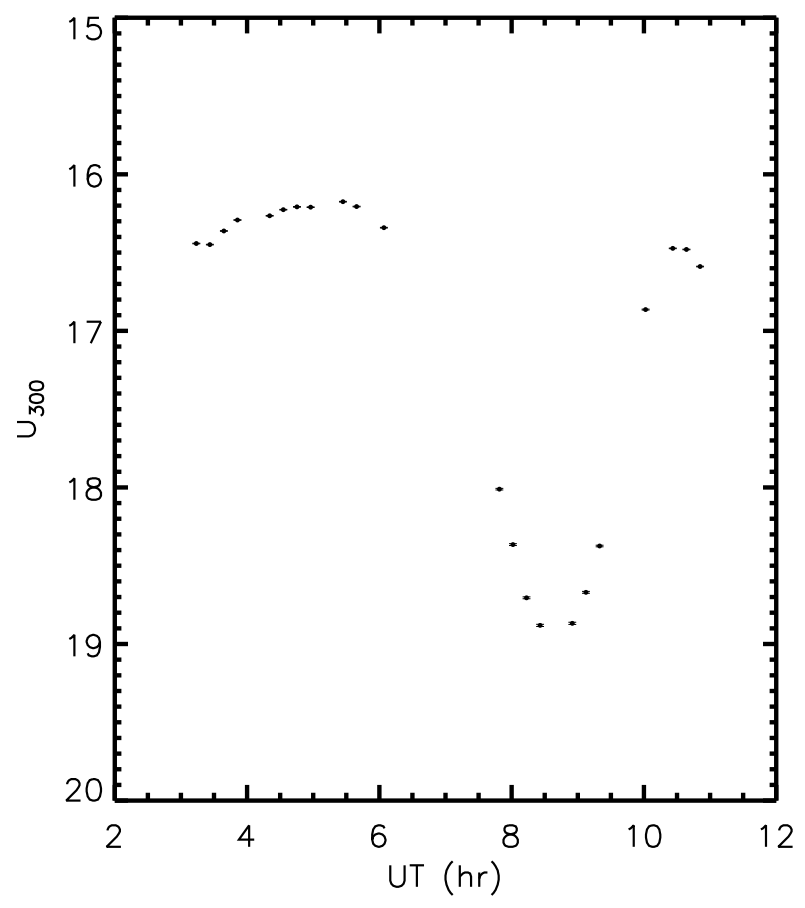

Figure 5. Dereddened partial light curve in the filter $\mathrm{U}_{300}$ of the of 1.1 day orbital period eclipsing CV AKO 9. Only photometric measurements that were labeled by DOLPHOT as good quality measurements are plotted.

change in behavior could be due to long-term changes in the system's configuration; or the component that dominates the NUV light is very different from the component that dominates the optical (e.g. disk versus mass donor).

The light curve of $\mathrm{W} 23_{U V}$ shows variability up to $\sim 2$ mag on a time scale of hours, as well (Figure 6). Examination of the individual images suggests that the observed variations are real. The NUV light curve of this object looks like it is declining back to a quiescent level, perhaps after a strong flare lasting at least several hours. Alternatively, we could have observed W23 right at the end of a DN outburst, which are recurrent brightenings of 2-5 mag observed in non-magnetic CVs. DN eruptions have already been detected in several 47 Tuc CVs (AKO 9, Auriere et al., 1989 W30 $U V$, Paresce \& de Marchi, 1994 and possibly W51 $1_{U V}$ and $\left.\mathrm{W} 56_{U V}, \mathrm{E} 03 \mathrm{~b}, \mathrm{H} 05\right)$. Unfortunately the time coverage of the $W 23_{U V}$ light curve is short and no more information can be obtained.

$\mathrm{W} 27_{U V}$ has already been noted as a CV displaying large-amplitude variability (Shara et al. 1996) and has been classified as a magnetic CV (H05) based on its periodic Xray light curve. Grindlay et al. (2001) and E03b proposed a possible orbital period of $3.83 \mathrm{hr}$ and $3.7 \mathrm{hr}$, respectively for this object (though Hattawi et al. -in preparation- have found a period of $6.7 \mathrm{hr}$ ). Our $\mathrm{U}_{300}$ phase-folded light curve using these periods does not show signs of periodicity. However, variations of $\sim 2.6 \mathrm{mag}$ in amplitude are visible in the light curve, which are similar to the ones observed in $\mathrm{W} 2_{U V}$.

Flickering variations in the optical light of CVs occur on time scales of $\sim 10 \mathrm{~min}$, and the corresponding brightness fluctuations around the mean value are not of the order of a few magnitudes but rather a few tenths of a magnitude. Flickering is therefore not a plausible explanation for the observed behavior of the systems discussed above. In Figure 6 we show our $\mathrm{U}_{300}$ light curve for $\mathrm{W} 30_{U V}$, in which the amplitude variations are likely due to flickering from the hot spot or accretion disk.

\subsection{A possible AM CVn system (W62) and an intriguing object (W21)}

The counterparts to the Chandra X-ray sources W21 and W62 are blue in the NUV and optical CMDs. Both objects have variability at a confidence level higher than $99 \%$ (Figure 4). Also, both objects lie very close to, or even slightly $\left(\lesssim 0.15 \mathrm{mag}\right.$ in $\left.\mathrm{H} \alpha-\mathrm{R}_{625}\right)$ to the right side of the $\mathrm{H} \alpha-\mathrm{R}_{625}$ MS. Compared to $\mathrm{W} 120_{U V}$, which also lies on that region, the inferred $\mathrm{H} \alpha$ emission for $\mathrm{W} 21_{U V}$ is much weaker, and perhaps even absent for W62 $U V$ (Figure 3). Witham et al. (2006) found that typical CVs show $\mathrm{H} \alpha$ emission lines with $|E W s|>10 \AA$, with $\mathrm{DN}$ in outburst the ones that usually have smaller EWs. However, the absence of large (several magnitudes) amplitude variations in the optical data of W21 and W62, which were taken at different epochs, argues against a DN nature. An intermediate polar nature could be a possibility, though spectroscopic observations of these kind of objects show that usually they exhibit $\mathrm{H} \alpha$ emission lines with EWs from tens to hundreds of $\AA$ (e.g. RX J0558+53 $|\mathrm{EW}(\mathrm{H} \alpha)|=604 \AA$, IGR J19267+1325 $|\mathrm{EW}(\mathrm{H} \alpha)|=120$ $\AA$, V2306 Cyg $|\mathrm{EW}(\mathrm{H} \alpha)|=35 \AA$; Harlaftis \& Horne 1999 . Steeghs et al. 2008. Witham et al.|2006) which contrasts with that estimated for $\mathrm{W} 62_{U V}$. Therefore, below we explore alternative explanations for $\mathrm{W} 21_{U V}$ and $\mathrm{W} 62_{U V}$.

$\mathrm{W} 21_{U V}$ is a periodic variable with an orbital period of 52 or 104 mins (E03b). The power spectrum and amplitude variations of this object favors the former period. However the folded light curve using the longer period is asymmetric, which could be due to ellipsoidal variations. If indeed the period is $52 \mathrm{~min}, \mathrm{~W} 21_{U V}$ should have a fully or partially degenerate secondary (the minimum period for CVs with a non degenerate donor is 75-80 mins; Gänsicke et al. 2009). $\mathrm{H}$-rich CVs with periods below 75 mins have been detected, examples are 485 Cen (59 min period, Augusteijn et al. 1996) and 1RXS J232953.9+062814 (64 min period, Thorstensen et al. 2002). However, these $\mathrm{CVs}$ have $\mathrm{EW}(\mathrm{H} \alpha) \sim-30 \AA$ and $-25 \AA$, respectively. Which are considerably larger than the determined $\mathrm{EW}(\mathrm{H} \alpha)$ for $\mathrm{W} 21_{U V}$. Moreover, these two short period CVs are $\sim 1$ absolute magnitude fainter than $\mathrm{W} 21_{U V}$ in $\mathrm{V}$ and $\mathrm{R}$, respectively. These characteristics argue against a very short period $\mathrm{H}$-rich $\mathrm{CV}$ nature for $\mathrm{W}_{2} 1_{U V}$. On the other hand, we think that the possibility for $\mathrm{W} 21_{U V}$ of being a system with a high accretion mass rate (nova-like) is low, since these systems are usually significantly brighter $\left(\mathrm{M}_{V} \lesssim 6.4 \mathrm{mag}\right.$, Warner 1987).

The object $\mathrm{W} 62_{U V}$ is the second brightest binary discussed in this paper (Figure 1). There are no observational constraints on the orbital period. The $\mathrm{EW}(\mathrm{H} \alpha)$ inferred from Figure 3 is small: $\mathrm{EW}(\mathrm{H} \alpha) \sim-3 \pm 6 \AA$, consistent with no $\mathrm{H} \alpha$ emission line.

We explored the possibility that $\mathrm{W} 21_{U V}$ and $\mathrm{W} 62_{U V}$ are double degenerate systems. In fact we found that the colors of $\mathrm{W} 62_{U V}$ are compatible with those obtained from AM CVn spectra (Figure 3) published by Carter et al. (2013). 

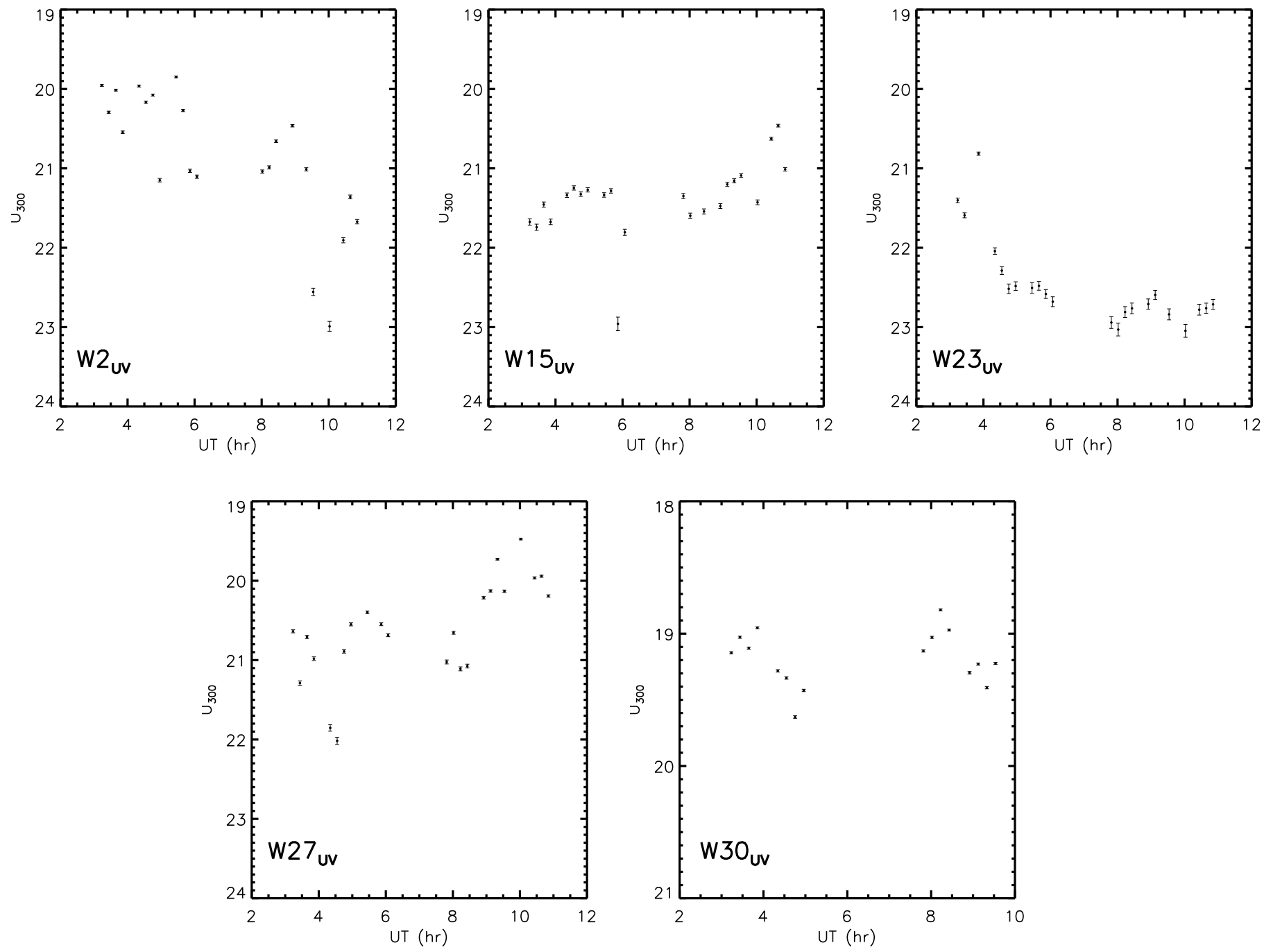

Figure 6. Dereddened light curves in the filter $\mathrm{U}_{300}$ of the $\mathrm{CVs} \mathrm{W} 2_{U V}, \mathrm{~W} 15_{U V}, \mathrm{~W} 23_{U V}, \mathrm{~W} 27_{U V}$ and $\mathrm{W} 30_{U V}$. The last is an already known dwarf nova system, added just for comparison matters. Large amplitude variations (more than 1 mag) are observed in the light curves of $\mathrm{W} 2{ }_{U V}, \mathrm{~W} 15_{U V}, \mathrm{~W} 23_{U V}$ and W27 $7_{U V}$ in time-scales of hours. Only photometric measurements that were flagged by DOLPHOT as good quality measurements are shown.

Moreover, if we estimate the period of $\mathrm{W} 62_{U V}$ using the magnitude-period relation for AM CVns given by Bildsten et al. (2006), we find that $\mathrm{W} 62_{U V}$ would have a period of $\sim$ 6 mins. Very similar to that of the known AM CVn system HM Cnc (Israel et al. 2002). Therefore, if W62 $U V$ would be confirmed as an AM CVn, then it is the first of that type detected in 47 Tuc and it is one of the few with an orbital period below 10 mins. The NUV color of $\mathrm{W} 62_{U V}\left(\mathrm{U}_{300}-\mathrm{B}_{390}\right.$ $\approx 0.15$ ) is consistent with those of AM CVn binaries (NUV$\mathrm{u}$ color goes from -0.8 to 0.7 ; Carter et al. 2013), and the same is true for its X-ray luminosity $\left(L_{X} \sim 10^{30}-10^{32}\right.$ erg $\mathrm{s}^{-1}$ for $\mathrm{AM}$ CVn binaries; Ramsay et al. 2006). We note that $\mathrm{W} 21_{U V}$ has an absolute $\mathrm{V}$ magnitude ( $\sim 7.4$ mags; E03a) that is not consistent $(\sim 5.5$ mag brighter $)$ with the one expected for an AM CVn system with an orbital period of $52 \mathrm{mins}$ (the $104 \mathrm{~min}$ period would automatically exclude $\mathrm{W} 21_{U V}$ from being a double degenerate). According to Bildsten et al. (2006), an AM CVn system with that period should have $\mathrm{M}_{V} \approx 13 \mathrm{mag}$ (as it has been observed for systems with similar periods, Solheim 2010).

Comparing the colors of $\mathrm{W} 62_{U V}$ and $\mathrm{W} 21_{U V}$ with those of He WD (like in Figure 9), they lie slightly to the right of the least massive cooling track. Together with the lack of radio emission, this gives evidence against a possible He WD MSP companion nature (but see Section 4.4). The probability of these objects of being black widow or redback systems is low, since the confirmed systems of that type in GCs have positions in the CMDs that are not consistent with those of $\mathrm{W} 62_{U V}$ or W21 $1_{U V}$ (see for example Edmonds et al. 2002, Cadelano et al. 2015). This is because black widow or redback systems lack an accretion disk and a hot, accreting WD. Instead, the optical flux comes from a (irradiated) secondary star, which causes the binary not to be as blue as $\mathrm{W} 62_{U V}$ or $\mathrm{W} 21_{U V}$ are. We discard a possible quiescence low mass X-ray binary (qLMXBs) nature for both objects, on account of their X-ray to ultraviolet flux ratio. Since CVs lack the deep potential wells of qLMXBs, they should gen- 


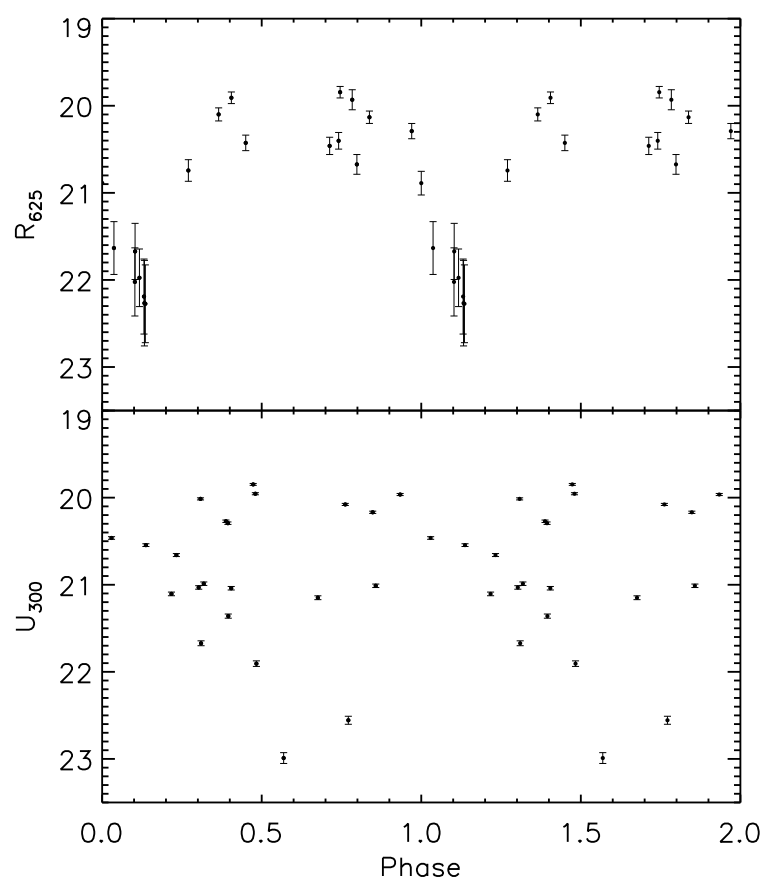

Figure 7. Phase-folded light curve in the $\mathrm{R}_{625}$ and $\mathrm{U}_{300}$ bands for the eclipsing CV W2 $U V$. We have used the period of $2.4 \mathrm{~h}$ given by Israel et al. (2016). The light curves have been dereddened but not barycentered. We used the same initial time when folding. The eclipse is clearly visible in $\mathrm{R}_{625}$ but not in $\mathrm{U}_{300}$. Note that the observations in $\mathrm{U}_{300}$ were taken at a different epoch than the observations in $\mathrm{R}_{625}$.

erate a much smaller ratio of X-ray to UV flux from material passing through their accretion disks. We found that the known qLMXBs in 47 Tuc have $\log \left(F_{X} / F_{300}\right)$ between 1 and 3 , while $\mathrm{W} 21_{U V}$ has a value of around -0.1 and $\mathrm{W} 62_{U V}$ $\sim-1.5$. Also, the positions of these two binaries in the NUV CMD do not match with those of qLMXBs in the cluster (Edmonds et al. 2002, van den Berg et al. in preparation). Given all the arguments mentioned above, the nature of the binary $\mathrm{W} 21_{U V}$ remains uncertain.

\subsection{Millisecond pulsar companions?}

We place in this group the counterparts to the X-ray sources W34, W70, W85 and W253. The first three objects were previously suggested to be either CVs or MSPs (Edmonds et al. 2002, E03a, E03b). However, our photometric results (especially the $\mathrm{H} \alpha$ ) argues against the CV nature (see Figure 3. . The X-ray source W253 is classified for the first time in this paper, and has been included in this section because of the similarities with W70 and W85. Figure 9 shows the position of these four binaries in the NUV CMD, and Table 2 contains their photometric and astrometric results.

W70 and W85 were previously discussed by E03a, E03b who found both objects with blue colors in the $\mathrm{U}-\mathrm{V}$ vs $\mathrm{U}$ and F300W - F555W vs F300W CMDs, respectively. However, a lack of I magnitude was mentioned by those authors for W70 $U V$, together with a mismatch of 2 pixels in the position of this object between the $\mathrm{V}$ and the $\mathrm{U}$ images. This mismatch is the result of the presence of a very close and bright

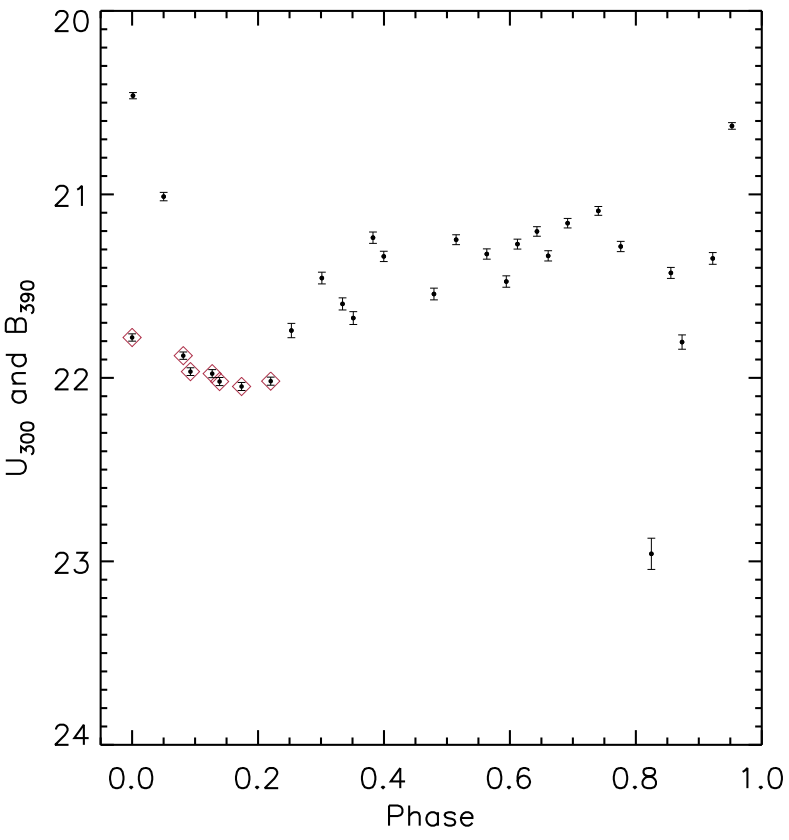

Figure 8. Dereddened phase-folded light curves in the filters $\mathrm{U}_{300}$ and $\mathrm{B}_{390}$ for the eclipsing $\mathrm{CV} \mathrm{W} 15_{U V}$. We have used the period of $4.238 \mathrm{~h}$ given by E03b. Red diamonds correspond to the observations in the filter $\mathrm{B}_{390}$. The eclipse is not visible in $\mathrm{U}_{300}$, instead variations of $\sim 2.5$ mag are observed. Only photometric measurements that were labeled by DOLPHOT as good quality measurements are plotted.

neighbor at only $0.12^{\prime \prime}$ from the real counterpart. Since in the NUV images both stars are clearly visible, reliable photometry for $\mathrm{W} 70_{U V}$ was obtained. The resulting magnitudes place this X-ray binary on the primary WD cooling sequence of the NUV and optical CMD. Its NUV, optical and $\mathrm{H} \alpha$ colors, indicate that $\mathrm{W} 70_{U V}$ could be a CO WD. Additionally its X-ray softness and lack of variability in the NUV, optical or X-ray emission are consistent with a (low state) magnetic $\mathrm{CV}$, a CO WD companion (it seems too blue to be an $\mathrm{He}$ $\mathrm{WD}$ ) to a so-far-undetected MSP, or a chance coincidence of an isolated WD within the X-ray error circle (see Section 4.5 .

The objects $\mathrm{W} 85_{U V}$ and $\mathrm{W} 253_{U V}$ show blue colors that place them between the primary WD sequence and the SMC MS. There are no indications of variability in any wavelength for these objects. In the optical CMD, W85UV appears on the primary WD sequence. This slight position change in the CMDs suggests that this binary is not an isolated WD matched by chance with the X-ray source. The object $\mathrm{W} 253_{U V}$ is between the WD sequence and the SMC MS in the optical CMD. However, we note that the presence of a bad column nearby might slightly affect the optical photometry. From the fitting of the X-ray spectra, H05 found that W253 is more suggestive of an MSP than a thermal plasma spectrum typical of CVs or ABs. Additionally, W253 ${ }_{U V}$ is almost at the center of the $3 \sigma$ error circle, which significantly reduces the probabilities of being a chance coincidence.

Given the results described above, we considered the possibility that $\mathrm{W} 85_{U V}$ and $\mathrm{W} 253_{U V}$ are faint CVs like 
Table 2. Astrometric and photometric results for the MSP companion candidates. Units of right ascension are hours, minutes, and seconds, and units of declination are degrees, arcminutes, and arcseconds. The photometric values are calibrated to the Vega-mag system. Photometric errors are given in parenthesis. The projected distance from the cluster center (r) was determined using the coordinates of the center of 47 Tuc as given by Goldsbury et al. (2010).

\begin{tabular}{ccccccccc} 
Source & R.A. & Decl $(\mathrm{J} 2000)$ & $\mathrm{U}_{300}$ & $\mathrm{~B}_{390}$ & $\mathrm{~B}_{435}$ & $\mathrm{H} \alpha$ & $\mathrm{R}_{625}$ & $\mathrm{r}\left({ }^{\prime}\right)$ \\
\hline $34_{U V}^{*}$ & $00: 24: 05.216$ & $-72: 04: 46.72$ & $23.8(2)$ & $23.59(9)$ & $24.1(1)$ & $22.5(1)$ & $23.2(2)$ & 0.10 \\
$70_{U V}$ & $00: 24: 11.874$ & $-72: 04: 44.21$ & $22.003(6)$ & $23.36(1)$ & $23.69(3)$ & $23.8(1)$ & $23.73(6)$ & 0.49 \\
$85_{U V}$ & $00: 23: 59.387$ & $-72: 04: 38.57$ & $22.545(9)$ & $23.17(1)$ & $23.44(5)$ & $23.7(2)$ & $23.4(1)$ & 0.54 \\
$253_{U V}$ & $00: 24: 10.983$ & $-72: 05: 04.99$ & $20.414(3)$ & $21.306(4)$ & $21.52(3)^{* *}$ & $21.6(1)^{* *}$ & $21.53(7)^{* *}$ & 0.45
\end{tabular}

* Mean photometric values.

** Photometry may be slightly affected by the presence of a bad column.

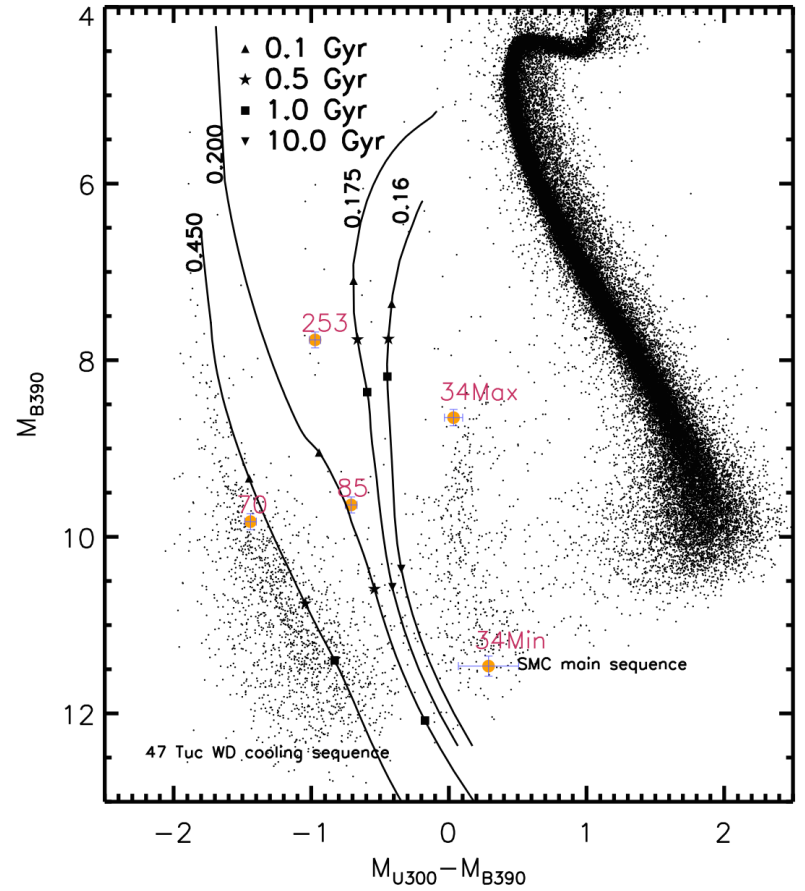

Figure 9. $U_{300}-B_{390}$ versus $B_{390}$ CMD based on DAOPHOT photometry of our NUV images. Each candidate MSP companion is denoted with the corresponding number and a filled orange circle. Calibrated magnitudes were converted to absolute magnitudes using a distance to 47 Tuc of $4.69 \mathrm{kpc}$ and reddening $E(B-V)=0.04$. The lines correspond to cooling models for $\mathrm{He}$ WDs with thick $\mathrm{H}$ envelopes as in Serenelli et al. (2002). They were computed for an initial metallicity of $Z=0.003$. The masses for which each track were calculated are indicated on the top of each track. The units are $M_{\odot}$.

$\mathrm{W} 120_{U V}$. However, from our Figure 3 we see that the colors of both binaries are indicative of the presence of prominent $\mathrm{H} \alpha$ absorption lines that are not typical of CVs, in contrast to the findings of $\mathrm{W} 120_{U V}$. Instead, their colors are in very good agreement with those of DA WDs (WDs with H-dominated atmospheres that show deep Balmer absorption lines) obtained from spectra published by Carter et al. (2013). We note that the lack of variability in W85UV and $\mathrm{W} 253_{U V}$ argues against an AM CVn nature. However, our results for these two objects are consistent with those described by Rivera-Sandoval et al. (2015) for He WD companions to MSPs in the cluster (see van Kerkwijk et al. 1996 Bassa et al. 2006, for more examples of (DA) WDs compan- ions to pulsars). The difference is that radio emission has not been reported either for $\mathrm{W} 85_{U V}$ or $\mathrm{W} 253_{U V}$.

Since $\mathrm{W} 70_{U V}, \mathrm{~W} 85_{U V}$ and $\mathrm{W} 253_{U V}$ have similar properties to the MSP companions, in Table 3 we show estimated masses, effective temperatures, surface gravities, luminosities, cooling ages, and X-ray properties for these three binaries assuming that they are indeed MSP companions. These parameters were obtained by comparison of He WD cooling models from Serenelli et al. (2002) to our NUV photometry. These models were computed for a metallicity $Z=0.003$, as appropriate for 47 Tuc. Using the absolute magnitudes in the filter $\mathrm{B}_{390}$ as independent variable, we have estimated the corresponding parameters by interpolating linearly along the two cooling tracks that bracket the location of W253 $3_{U V}$ and $\mathrm{W} 85_{U V}$. For $\mathrm{W} 70_{U V}$ the values were obtained by interpolating to the closest cooling track (that one for an He WD with $\left.0.45 M_{\odot}\right)$. As mentioned before, this object could be consistent with a CO WD with mass $>0.45 M_{\odot}$.

The counterpart to the X-ray source W34 is a short period variable with a period of $1.624 \mathrm{hr}$ (Edmonds et al.|2002). Light curves in the filters $\mathrm{U}_{300}$ and $\mathrm{B}_{390}$ folded on that period are shown in Figure 10, where we can observe the non sinusoidal behavior of $\mathrm{W} 34_{U V}$, with amplitude variations of $\sim 2.8$ magnitudes in the filter $\mathrm{B}_{390}$ and $\sim 3$ magnitudes in the filter $\mathrm{U}_{300}$. Only measurements labeled by DOLPHOT as good quality measurements were used. We note that the light curve of $\mathrm{W} 34_{U V}$ is not the typical light curve of a $\mathrm{CV}$ system. In fact, the light curve of $\mathrm{W} 34_{U V}$ matches in shape and amplitude those of radio MSPs with strongly irradiated companion stars in short orbits (such as 47 Tuc W Edmonds et al. 2002 PSR J1653-0158, Romani et al. 2014. XSS J12270-4859, Rivera Sandoval et al.|2017; and especially PSR B1957+20, Reynolds et al. 2007). W34 $4_{U V}$ displays a blue color in the NUV and optical CMDs (Figures 2 and 9. It shows small $\mathrm{H} \alpha$ excess, but the photometric errors in that band are considerable due to the presence of bright neighbors, thus it remains the possibility that this binary lies on the MS of the H $\alpha$ CMD. Edmonds et al. (2002) have already pointed out optical similarities between W34 $U V$ and the black widow system associated to W29 (47 Tuc W), with the difference that W34 has not been reported in radio wavelengths. Moreover, the strong heating from the light curves of $\mathrm{W} 34_{U V}$ proves that the intrinsic color of the companion is redder than the observed color. This rules out any kind of WD companion nature (as W3 $4_{U V}$ lies far to the right of the least massive He WD cooling track in Figure 9, suggesting that the companion is almost certainly a low-mass MS star. We also noted that this binary lies on the SMC MS. 
However, we discard a possible membership to that galaxy because at the distance of the SMC the source would be very bright $\left(2.6 \times 10^{33} \mathrm{erg} \mathrm{s}^{-1}\right)$, and it would have an orbital period far too short for being a SMC MS binary star.

As already mentioned, radio emission for all the systems discussed in this section has not been reported. That could be due to different reasons. The simplest one is that the direction of the radio beam is not pointed towards Earth. An alternative explanation is that the corresponding radio MSPs show disappearances (probably caused by clouds of material in the binary system) for several orbits that coincide with the time when the radio observations of the cluster have been taken, as occurs for PSR 1744-24A (e.g. Nice \& Thorsett 1992). Another explanation could be that they are faint radio sources compared to the other pulsars in the cluster, so they have been missed in the radio data analysis. Indeed, Pan et al. (2016) recently found two new isolated radio MSPs in 47 Tuc by reprocessing existing radio data with new methods. That opens the possibility of future detection (probably by more sensitive telescopes) of radio pulsations from the systems here discussed.

\subsection{Random coincidence test}

In a crowded cluster such as 47 Tuc it is important to take into account that some matches between X-ray sources and optical counterparts can be chance coincidences. In order to know how reliable the associations discussed in this paper are, we have carried out a test that computes the expected number of random coincidences between the X-ray sources and the NUV counterparts.

We first distinguish two regions in our NUV images: the core, limited by the core radius $\left(r_{c}=24^{\prime \prime}\right.$; Howell et al. 2000), and the region surrounding it but inside the NUV images. Of the $238 \mathrm{X}$-ray sources in our NUV FOV, 88 are in the core and 150 are outside the core but within the $\mathrm{r}_{h}$ of the cluster (see Figure A1 of Appendix A.

For the analysis, we followed the definition of blue object given at the beginning of Section 4. However we now call blue-gap object those stars found between the WD sequence and the MS of 47 Tuc ( $~ 1450$ objects $)$. In the same context, we define as WD an object that falls on the WD cooling sequence of the NUV CMD (Figure 1). Additionally, if an object shows NUV variability at a confidence level equal or higher than $95 \%$, we call it variable. Given that the number of blue-gap objects is smaller than WDs, we deduce that the probability of finding a blue-gap object within an $\mathrm{X}$-ray error circle is smaller than finding a WD. Additionally, we identified around 620 sources in the NUV FOV that show evident $\mathrm{Ha}$ excess $(\geqslant 99 \%$ confidence level) and are not associated to X-ray sources in the cluster.

We determined the density ( $\operatorname{stars} \operatorname{arcsec}^{-2}$ ) of variable (or non-variable) blue-gap objects inside and outside of the core radius of the cluster. We also determined the density of variable (or non-variable) WDs inside and outside of the core radius. Thus, we have four densities for variable objects and four densities for non variable objects. We have used these densities to calculate the total expected number of false matches within the $3 \sigma$ X-ray error circles of the 238 sources in our NUV FOV.

We expect $\sim 0.2$ false matches for variable blue-gap objects and the same number for variable WDs outside the core radius. On the other hand, we expect $\sim 0.8$ false matches for variable blue-gap objects and a similar value for variable WDs inside the core radius. Such values become much smaller if instead we consider the areas of the $1 \sigma$ and $2 \sigma$ $\mathrm{X}$-ray error circles, where many of the counterparts that we propose are.

For the non variable objects, we expect 10 (and 14) false matches for blue-gap objects (and WDs) outside the core radius ( $\sim 35 \%$ of the detected matches). On the other hand, we expect 13 (and 15) false matches for blue-gap objects (and WDs), inside the core radius ( $\sim 50 \%$ of the detected matches). These relative large numbers are not surprising, since within the $3 \sigma$ error circles of X-ray sources for which we have secure optical counterparts we have also found blue-gap or WD objects that are likely spurious alignments. Also, the total numbers of false matches are largely influenced by the sources that have large Xray error circles (e.g. many of the sources discussed in Section 4.1.5. It is possible that few of the non variable (and not detected in $\mathrm{H} \alpha$ ) $\mathrm{CV}$ candidates reported in this paper are part of these chance coincidences. These objects are $\mathrm{W} 24_{U V}, \mathrm{~W} 55_{U V}, \mathrm{~W} 75_{U V}, \mathrm{~W} 98_{U V}, \mathrm{~W} 100_{U V-\mathrm{a}}$ and -b, $\mathrm{W} 187_{U V}, \mathrm{~W} 202_{U V}, \mathrm{~W} 223_{U V}, \mathrm{~W} 229_{U V}, \mathrm{~W} 235_{U V}, \mathrm{~W} 302_{U V}$, $\mathrm{W} 324_{U V}, \mathrm{~W} 329_{U V}$ and W335 $U V$. However, the objects $\mathrm{W} 24_{U V}$ and $\mathrm{W} 302_{U V}$ are WDs that fall well inside the $1 \sigma$ error circle and are inside the core of the cluster, where the expected number of false matches among the 88 X-ray sources is 1.2. The counterparts to W229 and W253 (a proposed binary MSP also non-variable in NUV) are also within $1 \sigma$ but they lie outside the core of the cluster. The objects W98 $U V$, $\mathrm{W} 100_{U V-\mathrm{a}}$ and $-\mathrm{b}, \mathrm{W} 223_{U V}, \mathrm{~W} 235_{U V}$ and $\mathrm{W} 324_{U V}$, are inside the $1.5 \sigma$ error circle.

As discussed in previous sections, the counterparts to $\mathrm{W} 1, \mathrm{~W} 44$ and $\mathrm{W} 53$ are not variable in $\mathrm{U}_{300}$, but they actually are in other wavelengths (E03b). This suggests that most likely they are the correct counterparts to their Xrays sources and so the chance of being spurious matches is reduced. Among the MSP counterparts proposed (W34 $4_{U V}$, $\mathrm{W} 70_{U V}, \mathrm{~W} 85_{U V}$ and $\left.\mathrm{W} 253_{U V}\right)$, we think that $\mathrm{W} 70_{U V}$ has the largest chances to be a match coincidence. It was found at $3 \sigma$ and the counterpart does not show position changes in our different CMDs that could indicate that it is not a singletemperature WD. However, some of the confirmed MSP companions (also non-variable in NUV) by Rivera-Sandoval et al. (2015) present similar characteristics to $\mathrm{W} 70_{U V}$ and were also found at $3 \sigma$ from the X-ray source.

\section{COMPARISON TO OTHER GLOBULAR CLUSTERS: CORE-COLLAPSED VS NON CORE-COLLAPSED}

Given the results obtained in this paper, 47 Tuc is now the $\mathrm{GC}$ with the most CVs and CV candidates identified so far. It is followed in number of identified CVs and CV candidates by the cluster $\omega$ Cen (27 systems - Cool et al. 2013), NGC 6752 (16 systems - Lugger et al. 2017) and NGC 6397 (15 systems - Cohn et al. 2010). Characteristic parameters for these last two clusters are given in Table 1 and a list of the CVs and CV candidates found in them is given in Tables C1 and C2 of Appendix C The approximate X-ray limiting luminosities of these clusters are few times $10^{29} \mathrm{erg} \mathrm{s}^{-1}$. 
Table 3. Estimated masses $\left(\mathrm{M}_{c}\right)$, cooling ages, effective temperatures $\left(\mathrm{T}_{e f f}\right)$, surface gravities $(\log \mathrm{g})$, bolometric luminosities $(\mathrm{L})$ and X-ray luminosities $\left(L_{X}\right)$ for the possible counterparts to hidden MSPs in 47 Tuc. These parameters were obtained by comparing our NUV photometry to He WD cooling models with metallicity $Z=0.003$ (Serenelli et al. 2002).

\begin{tabular}{cccccccc} 
Source & $\begin{array}{c}\mathrm{M}_{c} \\
\left(\mathrm{M}_{\odot}\right)\end{array}$ & $\begin{array}{c}\mathrm{T}_{\text {eff }} \\
(\mathrm{kK})\end{array}$ & Log g & $\begin{array}{c}\text { Log L } \\
\left(L_{\odot}\right)\end{array}$ & $\begin{array}{c}\text { Cooling age } \\
(\mathrm{Gyr})\end{array}$ & $\begin{array}{c}L_{X} 0.5-6.0^{b} \\
\left(10^{30} \mathrm{ergs} \mathrm{s}^{-1}\right)\end{array}$ & $\begin{array}{c}L_{X} \\
\left(10^{30} \mathrm{ergs} \mathrm{s}^{-1}\right)\end{array}$ \\
\hline $34_{U V}$ & $<0.16$ & - & - & - & - & $16.5_{-1.1}^{+1.3}$ & $10.5_{-0.7}^{+0.8}$ \\
$70_{U V}$ & $>0.45$ & $>18.3$ & $>7.6$ & $>-1.4$ & $>0.18$ & $1.6_{-0.3}^{+0.4}$ & $1.5_{-0.2}^{+0.3}$ \\
$85_{U V}$ & $0.2-0.175$ & $11.4-9.2$ & $6.6-6.3$ & $-1.7--1.8$ & $0.2-3.8$ & $2.9_{-0.4}^{+0.6}$ & $1.7_{-0.3}^{+0.3}$ \\
$253_{U V}$ & $0.2-0.175$ & $11.7-9.9$ & $5.9-5.6$ & $-1.0--1.1$ & 0.5 & $2.7_{-0.4}^{+0.6}$ & $1.7_{-0.3}^{+0.3}$
\end{tabular}

${ }^{a}$ Ages represent the time between the point of highest $\mathrm{T}_{\text {eff }}$ on the cooling track until the observed point along the track.

$b$ Values taken from H05.
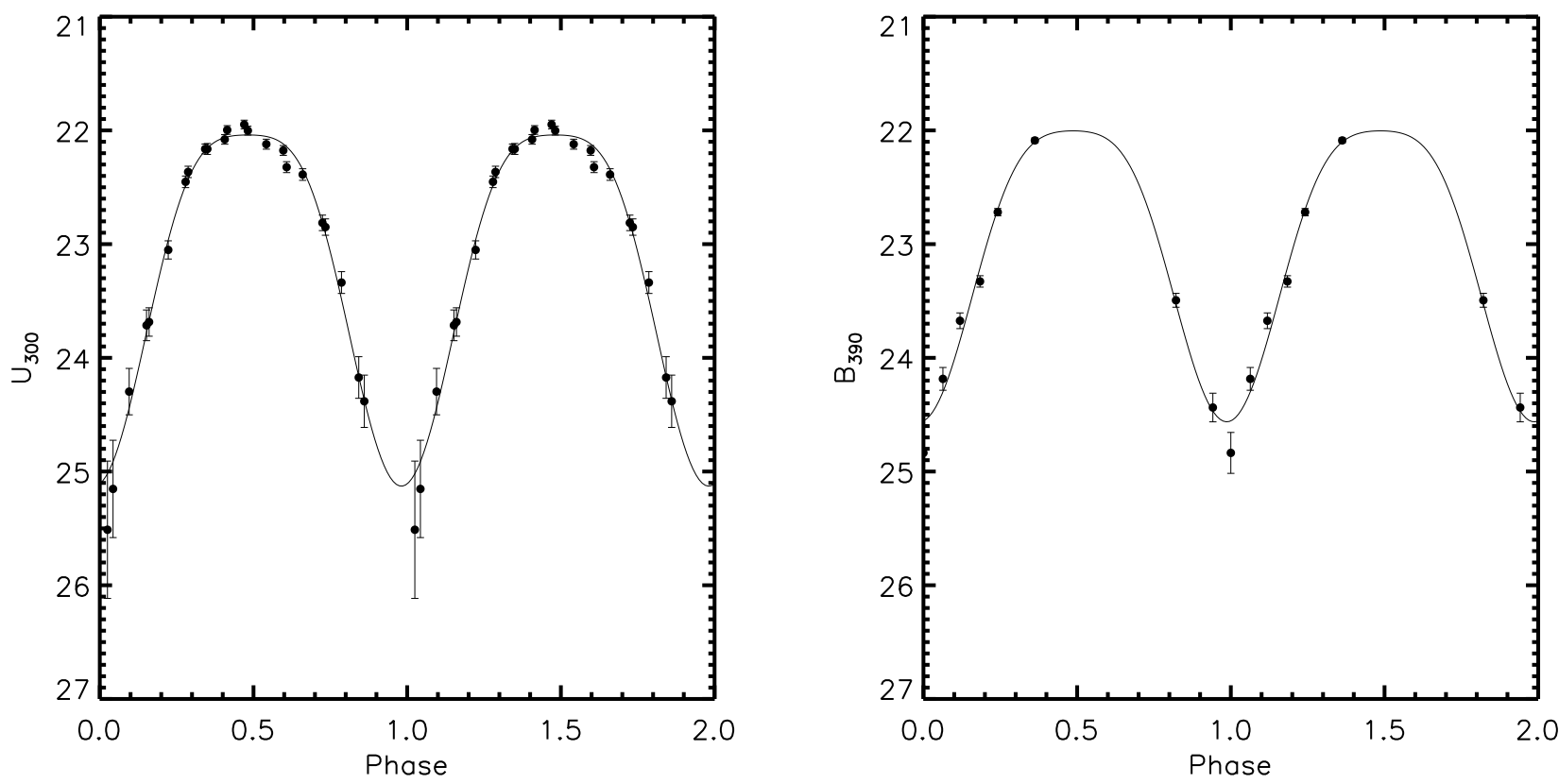

Figure 10. Folded light curves of $\mathrm{W} 34_{U V}$ in the filters $\mathrm{U}_{300}$ and $\mathrm{B}_{390}$. We have used a period of 1.624 hr. For clarity, the phase is plotted twice. The best fit is overplotted. The light curves are not similar to light curves of CVs, but they resemble more the light curves of systems in which the companion is strongly irradiated. The light curves are dereddened but not barycentered.

In the following sections we will compare the results found in 47 Tuc with the available results of the GCs NGC 6752 and NGC 6397 to obtain the best comparison of the $\mathrm{CV}$ population among the Galactic clusters. We exclude $\omega$ Cen from our comparison due to its remarkable differences, like its mass, its orbit and its velocity dispersion measure which suggest that $\omega$ Cen could be the disrupted core of a dwarf galaxy instead of a GC (see for example Majewski et al. 2000, Noyola et al. 2008). However, a comparison between the X-ray counterparts of $\omega$ Cen and the ones in NGC 6397, including the CV population, has been reported by Cool et al. (2013). They found a notable difference between the samples of both clusters. $\omega$ Cen (with a mass of $\left.40.5 \times 10^{5} M_{\odot}\right)$ has $\sim 3$ times more $\mathrm{CV}$ candidates in the range $L_{X}=10^{30}-10^{31} \mathrm{erg} \mathrm{s}^{-1}$ than NGC 6397, but similar number of CVs in the range $L_{X}=2 \times 10^{31}-2 \times 10^{32} \mathrm{erg}$ $\mathrm{s}^{-1}$. This suggests that unlike NGC 6397, the CV sample in $\omega$ Cen could be dominated by primordial CVs. We refer the interested reader to that paper for more details.
It is important to mention that in our comparison we only include the CVs and CV candidates discussed in Subsections 4.1 and 4.2. This is because the characteristics of the binaries $\mathrm{W} 34_{U V}, \mathrm{~W} 70_{U V}, \mathrm{~W} 85_{U V}$ and $\mathrm{W} 253_{U V}$ do not match those of typical $\mathrm{CVs}$, instead they match much better those of MSP companions. The nature of the sources $\mathrm{W} 21_{U V}$ and $\mathrm{W} 62_{U V}$ is not confirmed, and thus they have been excluded from the comparison as well. By excluding these sources, we can make a fairer comparison ${ }^{4}$ of the CVs with likely non degenerate donors between cluster's samples, since none of the CVs and CV candidates in NGC 6397 and NGC 6752 were suspected to be either AM CVn or MSP companions. For practicality, from now on we will refer as CVs to all CVs and CV candidates in the three clusters.

4 Note that NGC 6397 and NGC 6752 have not been studied in NUV. 


\subsection{X-ray and optical properties of the CV population}

Studies of the X-ray sources in the core-collapsed GCs NGC 6397 and NGC 6752 show that these clusters have two populations of CVs: a bright population (with apparent magnitudes $\left.\mathrm{R}_{625} \lesssim 21.5\right)$ that is more concentrated towards the core than a faint population $\left(\mathrm{R}_{625}>21.5\right)$, for which the WD dominates the brightness (Cohn et al. 2010, Lugger et al. 2017). When we compare the optical CMDs of these clusters with the optical CMD of the non core-collapsed cluster 47 Tuc, we find that in the last one we can not distinguish two groups of CVs as in NGC 6397 and NGC 6752 (Figure 2). For a better comparison, in Figure 11 we show normalized histograms of the absolute $\mathrm{B}_{435}$ magnitudes of the CVs with respect to the total number of identified CVs in each cluster. It is important to mention that the results shown in this histogram and in all the other histograms in this paper do not depend on the binning size. From Figure 11 we see that there are clear differences in the $\mathrm{B}_{435}$ luminosity distributions of the two types of clusters. We derived the probability density function of the absolute $\mathrm{B}_{435}$ magnitudes in the three clusters by carrying out a kernel density estimation (KDE, Parzen 1962) on our samples. Again, the shape of the KDE estimation does not depend on the binning size, but purely on the data. We chose a Gaussian function as kernel and a bandwidth given by the optimization approximation of Silverman (Silverman 1986). We found that 47 Tuc has an unimodal distribution, reaching the peak at absolute magnitude $\mathrm{M}_{B 435}=10$.

In Figure 12 we show the normalized luminosity function for 47 Tuc in the filter $\mathrm{U}_{300}$, which is more complete, since for some $\mathrm{CVs}$ the $\mathrm{B}_{435}$ magnitude could not be obtained. We observe that in the filter $\mathrm{U}_{300}$ the distribution is very similar to that in the filter $\mathrm{B}_{435}$. Since the shape of the distribution does not depend on the filter used, this suggests that 47 Tuc has an intrinsic unimodal distribution of CVs. On the other hand, the bimodal luminosity functions of NGC 6397 and NGC 6752 suggest that the corecollapsed clusters actually harbor more bright CVs than the non core-collapsed GC 47 Tuc. The difference between the distributions for non core-collapsed and core-collapsed clusters becomes more evident when we scale the number of CVs in NGC 6397 and NGC 6752 by the ratio between the mass of 47 Tuc and the mass of these clusters (Figure 13).

In all the figures discussed above we just excluded the counterpart to W100 since there is an ambiguity about which object is the correct counterpart (but even considering $\mathrm{W} 100_{U V-a}$ or $\mathrm{W}_{100_{U V-b}}$ the conclusions remain unchanged). Also, we noted that by excluding the counterparts to the X-ray sources W23, W24, W35, W75, W200, W202, W256, W299, W302, W304 and W335, which are classified as confused X-ray sources and X-ray sources with multiple potential counterparts (Section 4.1.5), the general results above mentioned do not change.

For the three clusters, we also compared the X-ray luminosity distributions of the CVs in the band $0.5-6 \mathrm{keV}$ (Figure 14. In order to avoid ambiguities in this comparison, we have removed eleven out of twelve X-ray sources that could be a blend of multiple nearby X-ray sources from the CV sample of 47 Tuc (see Section 4.1.5). However, we have included W100 in this analysis because we consider

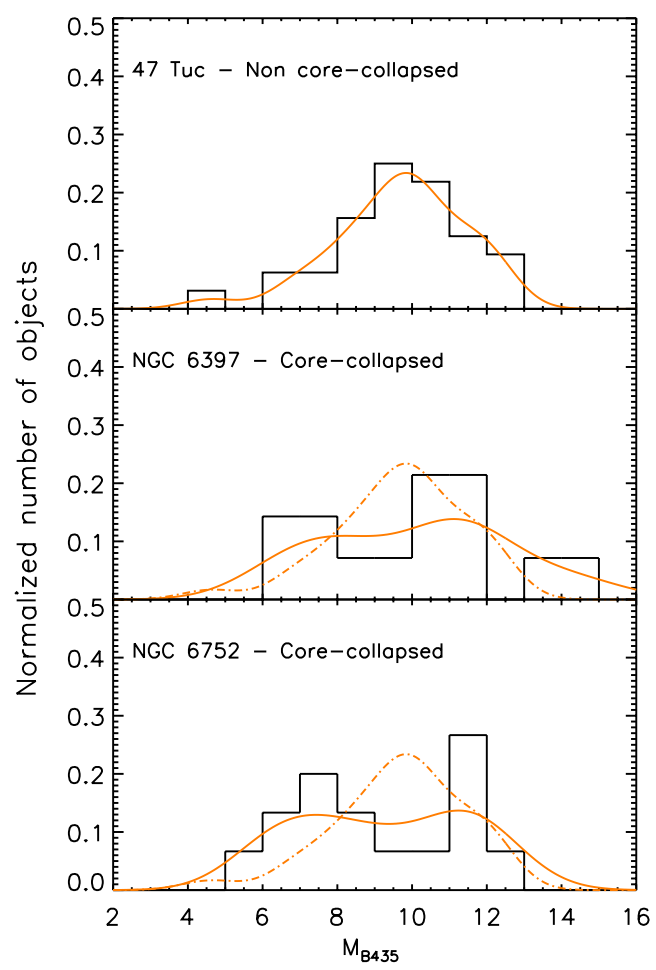

Figure 11. Normalized luminosity functions in the filter $\mathrm{B}_{435}$ for the CVs in 47 Tuc, NGC 6397 and NGC 6752 . In the case of 47 Tuc, only CVs for which we derived photometry in the $\mathrm{B}_{435}$ band are plotted. An exception is W100 which was excluded because the counterpart is ambiguous. The integral under the histogram is equal to 1 . In each plot, the orange line is the probability density function estimated by the KDE method using as kernel a Gaussian function. The dashed curves in all three plots represent the probability density function of 47 Tuc. The distribution function of 47 Tuc is unimodal, while the ones of NGC 6397 and NGC 6752 are bimodal.

that that X-ray source is the result of only one emitter (if we remove it, the gap of the X-ray CV distribution of 47 Tuc in Figure 14 becomes slightly more visible). For this cluster we have used the X-ray luminosities reported by H05. In the case of NGC 6752 we have transformed the X-ray luminosities given by Forestell et al. (2014) in the band $0.5-8 \mathrm{keV}$ to luminosities in the band $0.5-6 \mathrm{keV}$ by multiplying for the appropriate factor (0.95) assuming a $2 \mathrm{keV}$ bremsstrahlung model. For NGC 6397 we have converted the X-ray fluxes reported in Bogdanov et al. (2010) into X-ray luminosities assuming a distance of $2.2 \mathrm{kpc}$. In Figure 14 we show a histogram of X-ray luminosities of the CVs. We see that all the clusters present a bimodal distribution: a population of faint X-ray CVs $\left(L_{X} \lesssim 9 \times 10^{30} \mathrm{ergs} \mathrm{s}^{-1}\right)$ and another population of bright X-ray $\widetilde{C V s}_{\mathrm{S}}\left(L_{X}>9 \times 10^{30} \mathrm{ergs} \mathrm{s}^{-1}\right)$. This could be explained by the mass transfer rates in CVs, the higher the mass transfer rate, the higher the $\mathrm{X}$-ray luminosity. The gap observed in all the clusters could be related to the CV period gap, since objects below the period gap would have lower mass transfer rates compared to CVs above it (Howell et al. 2001). 


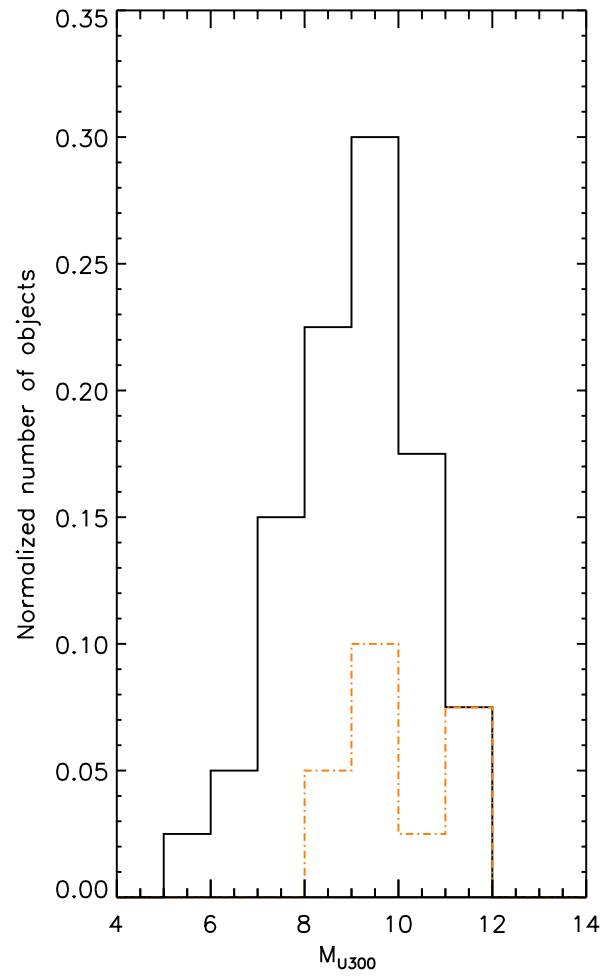

Figure 12. Normalized luminosity function in the filter $\mathrm{U}_{300}$ for the CVs in 47 Tuc. This cluster shows an unimodal distribution. Almost all the CVs discussed in this paper are included in this plot. Exceptions are the counterparts to W100, W120 and W122. The first one has an ambiguous counterpart. The last two are outside of the NUV FOV. The integral under the solid line histogram is equal to 1 . The dashed line histogram represents the CVs for which $\mathrm{M}_{B 435}$ was not obtained.

\subsection{Spatial distribution}

Assuming that the center of 47 Tuc corresponds to that reported by Goldsbury et al. (2010), which is R.A.= 00:24:05.71, Decl. $=-72: 04: 52.7$ in epoch J2000, we have calculated the projected radial distance to each CV in the cluster using that reference point. Results of the projected distance are given in column 4 of Table 5 In Figure 16 we have a histogram of the distribution of CVs with respect to the corresponding center of 47 Tuc, NGC 6752 and NGC 6397. The projected radial distributions have been normalized by the $\mathrm{r}_{h}$ of each cluster. For 47 Tuc, NGC 6752 and NGC 6397 the respective $\mathrm{r}_{h}$ values are 2'.79, 1'.91 and 2'33. Our NUV images of 47 Tuc cover a radial distance equivalent to $50 \%$ of its $\mathrm{r}_{h}$ (see Appendix A). At that distance we cover around $80 \%$ of the total number of X-ray sources detected within the $\mathrm{r}_{h}$ (see Figure 15). However, H05 showed that at radial distances larger than $\sim 100^{\prime \prime}$, most of the $\mathrm{X}$-ray sources identified are consistent with background objects. This means that if we discard these sources, our NUV images cover $\sim 95 \%$ of the X-ray objects in 47 Tuc within $\mathrm{r}_{h}$ (see Figure 8 of H05).

From Figure 16 we observe that apart from the different number of CVs detected in each cluster, the three clusters also show different radial distributions. In 47 Tuc, the number of CVs decreases gradually as we get far from the core. A different radial distribution is observed for the core-collapsed

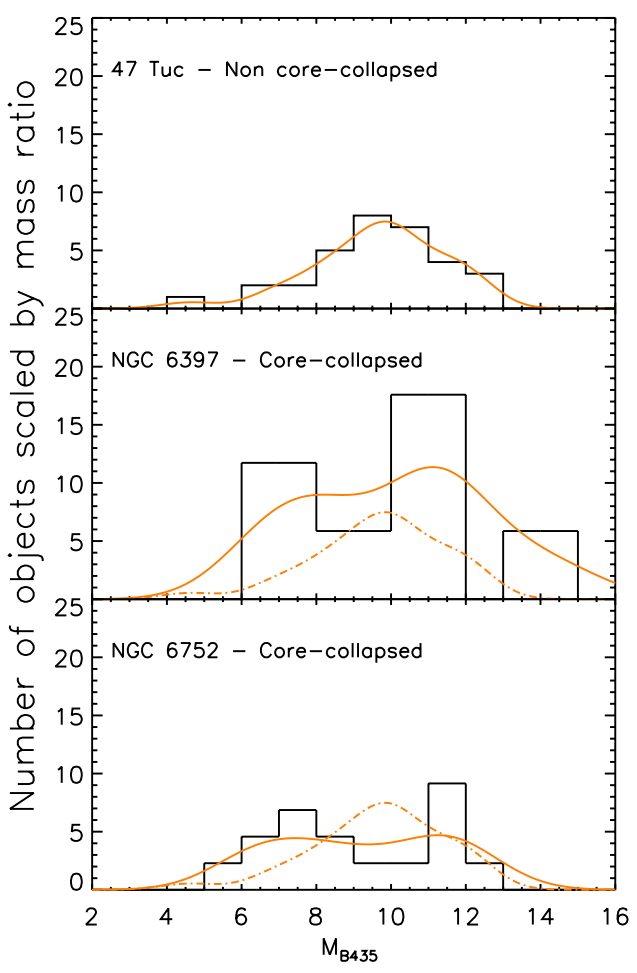

Figure 13. Mass scaled luminosity functions in the filter $\mathrm{B}_{435}$ for the CVs in 47 Tuc, NGC 6397 and NGC 6752. The corresponding scale is the ratio between the mass of 47 Tuc and the mass of these clusters. In the case of 47 Tuc, only CVs for which we derived photometry in the $\mathrm{B}_{435}$ band are plotted. An exception is W100 which counterpart is ambiguous and so it was excluded. In each plot, the orange line is the probability density function estimated by the KDE method using as kernel a Gaussian function. The dashed curves in all three plots represent the probability density function of 47 Tuc. The distribution function of 47 Tuc is unimodal, while the ones of NGC 6397 and NGC 6752 are bimodal. Note that core-collapsed clusters have a larger number of CVs with absolute magnitudes $\mathrm{M}_{B 435} \leqslant 8$ than 47 Tuc.

clusters NGC 6752 and NGC 6397 for which two groups are distinguishable: a large group of CVs more concentrated in the cores of these clusters, and a small group at larger radii, as has been already pointed out by Cohn et al. (2010) and Lugger et al. (2017). We carried out a Kuiper test (which is as sensitive in the tails as at the median of the distribution; Kuiper 1960 to know which is the probability that the radial distributions of NGC 6397 and NGC 6752 are drawn from the same radial distribution as 47 Tuc. We obtained a probability of $7.2 \%$ between 47 Tuc and NGC 6397, and a probability of $28 \%$ between 47 Tuc and NGC 6752 . This relatively large percentage could be due to the small CV sample at $r / r_{h}>0.4$ in NGC 6752 . The probability obtained between NGC 6397 and NGC 6752 is $74 \%$.

In Section 5.1 we showed that the CVs in the three clusters have a bimodal distribution in X-rays. In Figure 17 we show a plot of $L_{X}(0.5-6 \mathrm{keV})$ vs $r / r_{h}$ for the three clusters. We note that at $r / r_{h} \gtrsim 0.5$ most of the CVs in all the clusters have X-ray luminosities below $2 \times 10^{31}$ erg $\mathrm{s}^{-1}$. This can be explained by faint and old CVs that have 


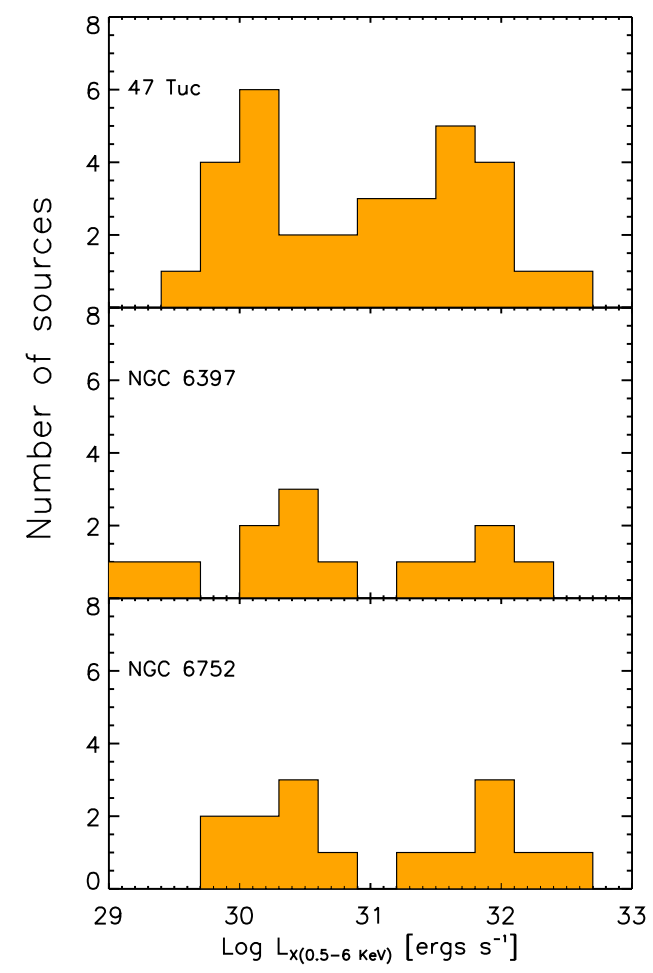

Figure 14. X-ray luminosity function for the CVs in the globular clusters 47 Tuc, NGC 6397 and NGC 6752. All the clusters show a bimodal distribution. For 47 Tuc, only X-ray sources with one possible NUV/optical counterpart are plotted. The three clusters have an X-ray limiting luminosity of $\sim 10^{29} \mathrm{erg} \mathrm{s}^{-1}$.

been ejected to larger radii due to stellar interactions in the clusters.

For 47 Tuc, we also analyzed the radial distribution of the CVs following the procedures described in Cohn et al. (2010) and Lugger et al. (2017) for fitting a parameterized surface-density profile to a main-sequence turnoff (MSTO) sample and subsamples of the CV population. This produces an estimate of the characteristic mass for all of the CVs taken together, and for the bright - and faint - CV subsamples. The KS2 photometry was used for this analysis. We first determined the cluster center by iterative centroiding in a $30^{\prime \prime}$ aperture, using a MSTO sample defined by $17.7 \leqslant B_{390} \leqslant 20.2$. The resulting center coordinates of R.A. $=00: 24: 05.76$, Decl. $=-72: 04: 52.6$ agree well with the definitive center determination by Goldsbury et al. (2010), differing by only $0^{\prime \prime} 2$.

We next determined the cumulative radial distribution of a MSTO sample that extends out to the nearest edge of the mosaic frame, which is $80^{\prime \prime}$ from the cluster center. We fitted the cumulative radial distribution of the MSTO group with a "generalized King model", which we have also called a "cored power law". As discussed by Cohn et al. (2010), this takes the form,

$$
S(r)=S_{0}\left[1+\left(\frac{r}{r_{0}}\right)^{2}\right]^{\alpha / 2}
$$

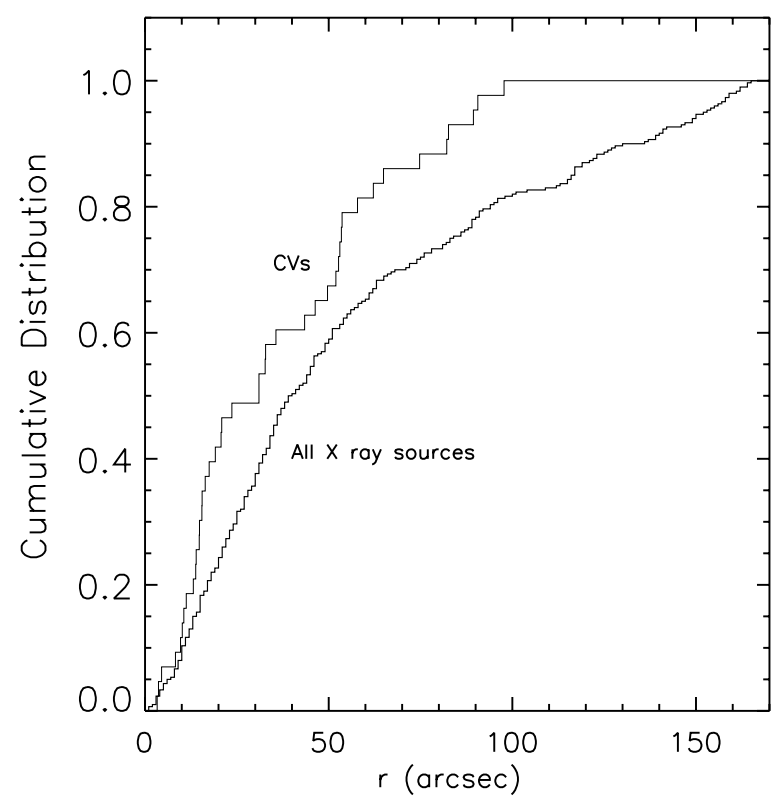

Figure 15. Cumulative radial distribution of the CVs and all the X-ray sources in the catalog of H05. All the CVs detected are within $100^{\prime \prime}$, where $\sim 80 \%$ of all the X-ray sources detected by H05 reside. X-ray sources with $r>100^{\prime \prime}$ are more consistent with background objects.

with the core radius related to the scale parameter $r_{0}$ by,

$$
r_{c}=\left(2^{-2 / \alpha}-1\right)^{1 / 2} r_{0}
$$

The result of the maximum-likelihood fit of this profile to the MSTO sample is given in Table 4 We note that the best-fit core radius value, $25^{\prime \prime} .1 \pm 0$.' 6 agrees well with the value of $24^{\prime \prime} .0 \pm 1^{\prime \prime} .9$ determined by Howell et al. (2000). The best-fit value of the slope parameter, $\alpha=-1.6$, falls close to the value of -1.8 that we previously found to give a good fit to the central profile of a King $(1966)$ model with a concentration parameter of $c=2$ (Lugger et al. 1995). This supports the conclusion by Howell et al. (2000) that 47 Tuc has a large, normal core and is not presently undergoing core collapse.

We next examined the radial profile of the CVs, taken both as a single sample and as separate bright and faint samples. We adopted a separating magnitude between the bright and faint samples of $\mathrm{M}_{B 390}=9.0$. The limit was chosen to be consistent with the magnitude limits found observationally in NGC 6397 and NGC 6752. The cumulative radial distributions of these samples are plotted in Figure 19 From that figure we see that the population of CVs in the cluster is much more centrally concentrated than the MSTO population, in agreement with numerical models of Hong et al. (2017). This concentration of CVs in the cluster could be due either to their production in the core and/or to their higher average mass compared to other stars. However, the fact that the brighter CVs (which must be relatively young) are not substantially more centrally concentrated than the fainter $\mathrm{CVs}$, indicates that the central concentration of CVs 


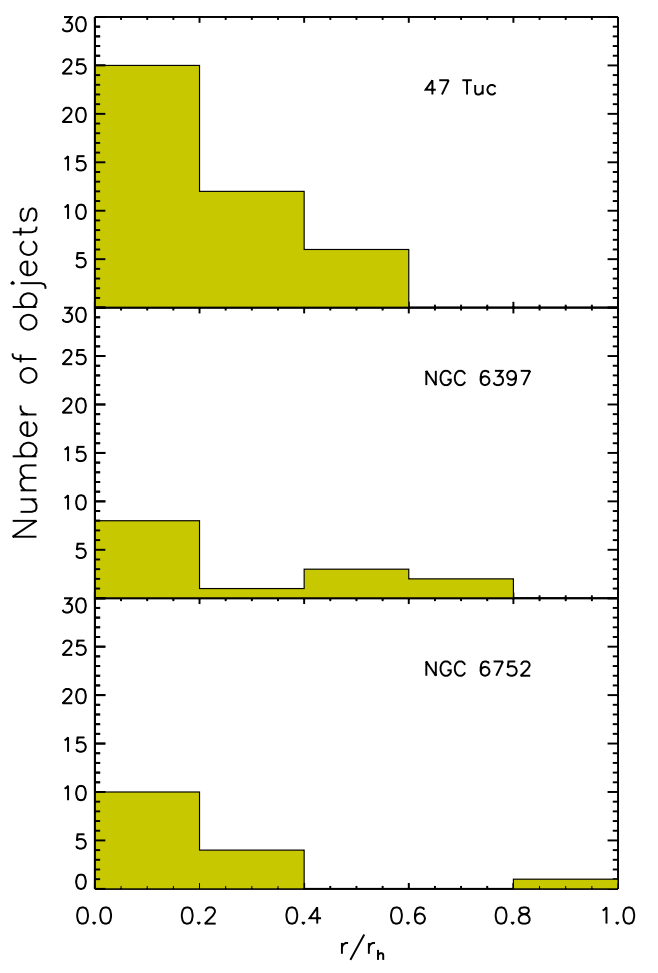

Figure 16. Histogram of the normalized radial distance of $\mathrm{CV}$ identifications in the globular clusters 47 Tuc, NGC 6397 and NGC 6752. The last two clusters show a second group at larger radii. For each cluster, the projected radial distance $(r)$ has been normalized by the corresponding half-mass radius $\left(\mathrm{r}_{h}\right)$. The green color denotes the total number of objects per bin.

is caused more by their higher total masses than by their place of formation. This may indicate that both the bright and faint CVs are old enough to have relaxed to their equilibrium radial distribution. We then quantified the CV masses by carrying out maximum-likelihood fits of the cored-powerlaw model to three samples (all, bright and faint CVs). As discussed by Cohn et al. (2010), this procedure provides an estimate for the characteristic mass of an object in each group relative to the MSTO mass. As in Cohn et al. (2010), we adopt the approximation that the mass groups above the MSTO mass are in thermal equilibrium. In this case, the surface-density profile for a mass group with mass $m$ is given by Eqn. 1 1 with a slope parameter $\alpha$ related to the turnoff-mass slope $\alpha_{\text {to }}$ by,

$$
\alpha=q\left(\alpha_{\text {to }}-1\right)+1
$$

where $q=m / m_{\text {to }}$, with $m_{\text {to }}$ the average mass of the MSTO stars and $m$ is the mass of the binaries (in this case of the CVs).

Table 4 gives the results of maximum-likelihood fits of Eqn. 1 to the samples formed by the turnoff-mass stars, all CVs, bright CVs, and faint CVs. In all cases, we only consider objects that lie within $80^{\prime \prime}$ of the cluster center, to exclude incomplete annuli that extend into the corners of the mosaic. Figure 18 shows a binned radial profile for

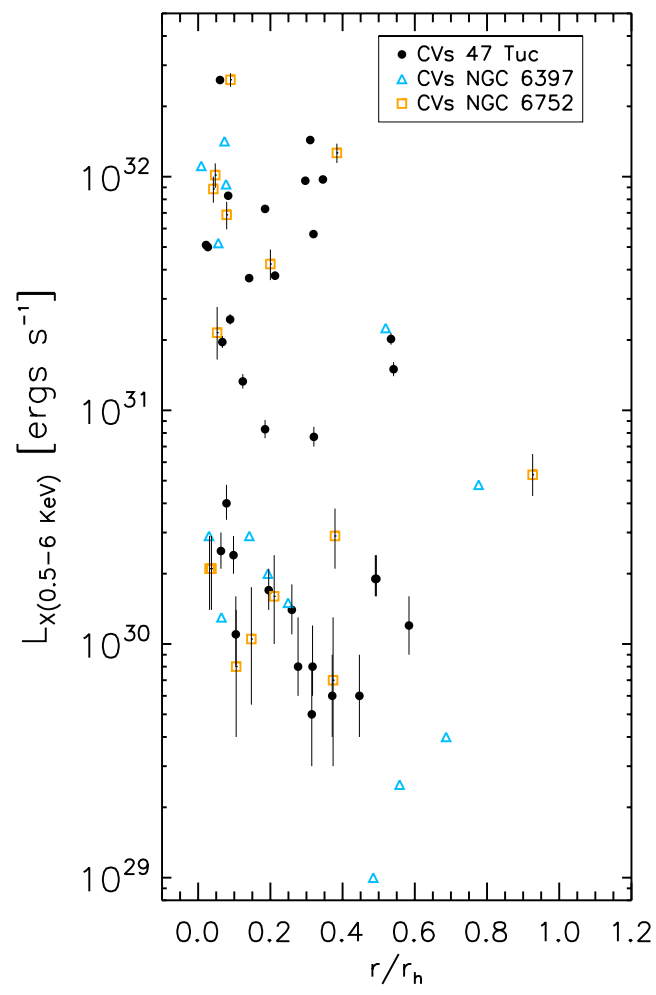

Figure 17. Normalized radial distribution vs X-ray luminosity for the CVs in the globular clusters 47 Tuc, NGC 6397 and NGC 6752. For 47 Tuc, only X-ray sources (plus W100) that are not part of the X-ray sources with multiple NUV/optical counterparts are plotted. In all the clusters we see that at $r / r_{h} \sim 0.5$ the CVs have X-ray luminosities below $2 \times 10^{31} \mathrm{erg} \mathrm{s}^{-1}$. X-ray luminosities for the CVs in NGC 6397 were derived from Bogdanov et al. (2010). However, errors are not specified by those authors.

the MSTO group with the cored-power-law fit. As can be seen from the table, the $q$ values for the three CV samples exceed unity, indicating that the characteristic masses indeed exceed the turnoff mass. We assume a MSTO mass of $0.85 \pm 0.05 M_{\odot}$, based on the study of Ferraro et al. (2016). For the all-CV and faint-CV samples, the excesses are significant at the $\sim 3 \sigma$ level, while for the smaller bright-CV sample, the significance of the excess is nearly $2 \sigma$. As found in the analysis described above, there is no apparent difference between the characteristic masses of the bright and faint CVs.

The inferred characteristic mass for the all-CV sample of $1.4 \pm 0.2 M_{\odot}$ is similar to what has been found in the core-collapsed clusters NGC 6397 (Cohn et al. 2010) and NGC 6752 (Lugger et al. 2017). For 47 Tuc, the median absolute magnitude of the bright $\mathrm{CVs}$ is about $M_{R} \approx 7.0$. Assuming that most of this flux is contributed by the main sequence secondary, the mass of the secondary is about $0.55 M_{\odot}$, based on stellar evolutionary models of Baraffe et al. (1997) (this is consistent with the mass derived for the object $\mathrm{W} 23_{U V}$ in Section 4.1.4. This implies a WD mass of $0.9 M_{\odot}$. We note that this is consistent with the mean WD mass of $0.83 \pm 0.23 M_{\odot}$ obtained by Zorotovic et al. (2011) for field CVs in the Sloan Digital Sky Survey (SDSS). For the faint CVs, in which the optical flux is dominated by the WD and accretion disk, the secondary mass is likely to be 
Table 4. Cored-Power-Law model fits to $80^{\prime \prime}$ from the cluster center. the parameter $\mathrm{N}$ is the number of stars in each sample within $80^{\prime \prime}$ of cluster center, $q$ is the mass ratio, $r_{c}$ is the core radius, $\alpha$ is the slope parameter, $m$ is the mass of the MSTO stars or of the CVs, $\sigma$ is the significance of mass excess above MSTO mass, and finally the parameter $\mathrm{p}$ is the K-S probability of consistency with the MSTO group.

\begin{tabular}{cccccccc} 
Sample & $\mathrm{N}$ & $\mathrm{q}$ & $\mathrm{r}_{c}\left({ }^{\prime \prime}\right)$ & $\alpha$ & $m\left(M_{\odot}\right)$ & $\sigma$ & $\mathrm{p}$ \\
\hline MSTO & 34358 & 1.0 & $25.1 \pm 0.6$ & $-1.58 \pm 0.04$ & $0.85 \pm 0.05$ & - & - \\
all CVs & 36 & $1.63 \pm 0.20$ & $15.6 \pm 1.5$ & $-3.21 \pm 0.51$ & $1.39 \pm 0.17$ & 3.0 & $0.067 \%$ \\
bright CVs & 11 & $1.70 \pm 0.36$ & $15.1 \pm 2.6$ & $-3.39 \pm 0.94$ & $1.45 \pm 0.31$ & 1.9 & $9.7 \%$ \\
faint CVs & 25 & $1.60 \pm 0.24$ & $15.8 \pm 2.0$ & $-3.14 \pm 0.62$ & $1.36 \pm 0.20$ & 2.5 & $1.0 \%$
\end{tabular}

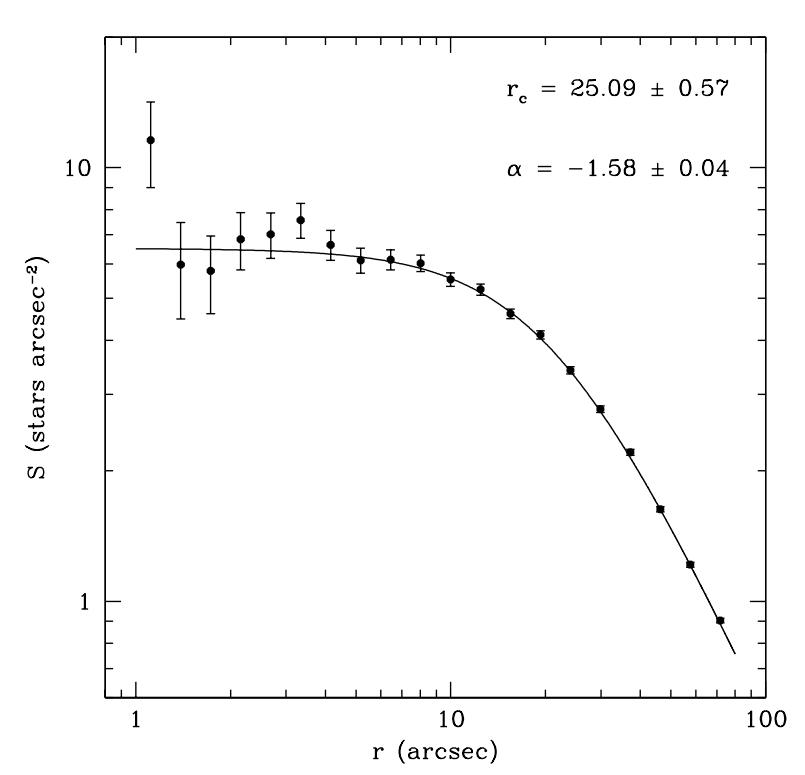

Figure 18. Binned radial surface-density profile for MSTO group with cored-power-law fit. The model gives a good fit to all but the innermost bin, which is the most subject to small-number statistics.

$\lesssim 0.2 M_{\odot}$, assuming that the secondary is a main sequence star. In this case, the inferred typical WD mass is $\sim 1.2 M_{\odot}$. This suggests a scenario in which there is a net increase in WD mass in CVs over the combined action of accretion and nova outbursts, as has been investigated by Wijnen et al. (2015). Alternatively, the high system masses that we infer for the faint CVs in 47 Tuc may, at least in part, represent a selection effect. If we are most sensitive to the most X-ray bright sample of a larger population of faint CVs, then we may be biased towards detecting the high-WD-mass end of the population, since larger WD masses (and concomitantly smaller radii) lead to deeper potential wells that generate more X-rays for a given mass accretion rate.

To complement this analysis, we performed the Kolmogorov-Smirnov (K-S) comparison of the CV samples to the MSTO sample, restricting the samples to objects that are within $80^{\prime \prime}$ of the cluster center. The resulting cumulative distributions for the present analysis are shown in Figure 19 and the K-S probability values are given in Table 4 The results of this KS analysis are similar to the results of the profile fitting. Both the all-CV and faint-CV samples are found to have significantly more centrally concentrated distributions than that of the MSTO sample.

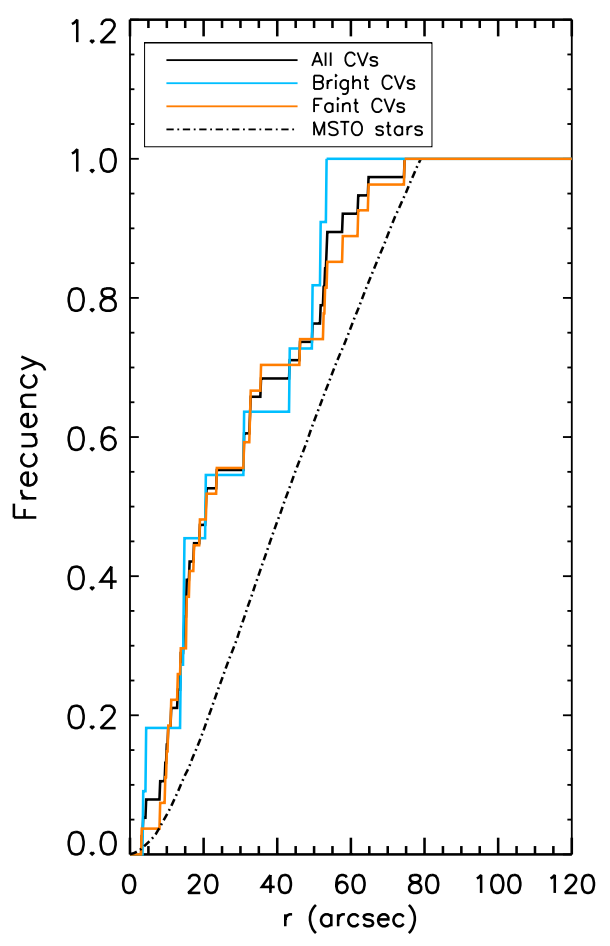

Figure 19. Cumulative radial distributions for selected stellar groups, within $80^{\prime \prime}$ of the cluster center. Note that the all-CV, bright-CV, and faint-CV groups all show central concentration relative to the MSTO group.

\subsection{The influence of stellar interactions}

Our results and comparisons suggest that core-collapsed GCs, in terms of CVs, behave optically different than 47 Tuc which is non core-collapsed. Of course, more identifications of CVs in both types of clusters are necessary in order to have better statistics. However, our comparison gives for the first time suggestions about the observational differences between the two kind of clusters.

For a long time it has been debated whether the corecollapse process in GCs affects the creation and destruction of CVs. Observations of the core-collapsed clusters NGC 6397 and NGC 6752 are consistent with an evolutionary scenario in which dynamical interactions have a stronger effect in the creation of these binaries near the center of the cluster. This explains the brighter and more concentrated population of CVs. When these CVs become old and faint they scatter to larger radii due to interactions with other stars in the cluster, in agreement with the observed less bright population (Cohn et al. 2010, Lugger et al. 2017). 
The population of CVs in 47 Tuc is dominated by faint systems, suggesting that there are more primordial and/or old dynamically formed CVs in comparison to NGC 6397 and NGC 6752. The bright CVs in 47 Tuc were probably created a few Gyrs ago, but clearly in less amount than in the core-collapsed clusters. This is consistent with evolutionary models of CVs which indicate that, since dynamicallyformed CVs generally have relatively massive secondaries, brighter CVs have generally been produced more recently than fainter ones (e.g Belloni et al. 2017b).

According to the CV evolutionary models of Ivanova et al. (2006) that are relevant for 47 Tuc (results of their Table 3 multiplied by a factor of 1.3 , considering a core mass for 47 Tuc of $\left.6.5 \times 10^{4} M_{\odot}\right), \sim 276-287$ CVs should currently ${ }^{5}$ be present in the cluster. Of these CVs, 53-66 are predicted to be detectable, for which Ivanova et al. (2006) required that $L_{X}>3 \times 10^{30} \mathrm{erg} \mathrm{s}^{-1}$, and the donor stellar luminosity $>0.06 L_{\odot}$, such that the $\mathrm{CV}$ could be detected by both Chandra and HST. In Section 4.1.5 we mentioned that due to the high stellar crowding in 47 Tuc, some X-ray emitters can lie too close to each other and therefore remain unresolved by Chandra. H05 estimated that around $10 \%$ of the $\mathrm{X}$-ray sources detected within the $\mathrm{r}_{h}$ could be blends. This implies that a similar percentage of CVs detected through $\mathrm{X}$-ray emission could be missing. It is also possible that some CVs could have landed on bright stars, thus being undetected. Additionally, our limiting magnitudes in X-rays $\left(3 \times 10^{29} \mathrm{erg} / \mathrm{s}\right.$ in the band $\left.0.5-6 \mathrm{keV}\right)$ and in NUV/optical $(\sim 27.5$ in NUV) prevent us to detect CVs that are fainter than these limits. However, the predicted number of "detectable" CVs by Ivanova et al. (2006) is in good agreement with the $43 \mathrm{CVs}$ that we have detected in 47 Tuc, though note that neither the X-ray nor the optical/UV flux can be accurately predicted for real (highly variable) CVs, so the Ivanova et al. (2006) model predictions should be taken as rough indicators (Figure 20 shows a comparison between our detections and the models of Ivanova et al. 2006). Ivanova et al. (2006) also found that at 11-12 Gyrs, 30-34\% of the CVs (corresponding to $\sim 15$ of the identified CVs in 47 Tuc), were descendants of primordial binaries (though a significant fraction of these primordial CVs have been affected by encounters, e.g. by increasing their eccentricity). Also, 35-39\% of CVs were formed through binary exchange encounters, the largest channel for production of CVs. Binary collisions (13\%), tidal captures (3\%), and direct collisions of stars with red giants $(15 \%)$ offer alternative dynamical formation mechanisms for CVs in these models.

Shara \& Hurley (2006), and Belloni et al. (2016, 2017 $2017 \mathrm{~b}$ ) also found that the creation of exchange binaries is more common in dense environments. These exchange interactions are likely to make CVs with MS star masses $<0.8$ $\mathrm{M}_{\odot}$ (Belloni et al. 2017b), which tend to be brighter and shorter-lived than CVs in non-cluster environments. Ivanova et al. (2006) pointed out that the formation of CV progenitor binaries in high-density environments happens a couple of Gyrs before they appear as CVs, and that immediately after a substantial increase in the density of the cluster core, some existing CVs are dynamically destroyed while CV progenitors that are just being created are not yet bright. Thus, the

\footnotetext{
5 At an age of 11-12 Gyr, approximate age of 47 Tuc.
}

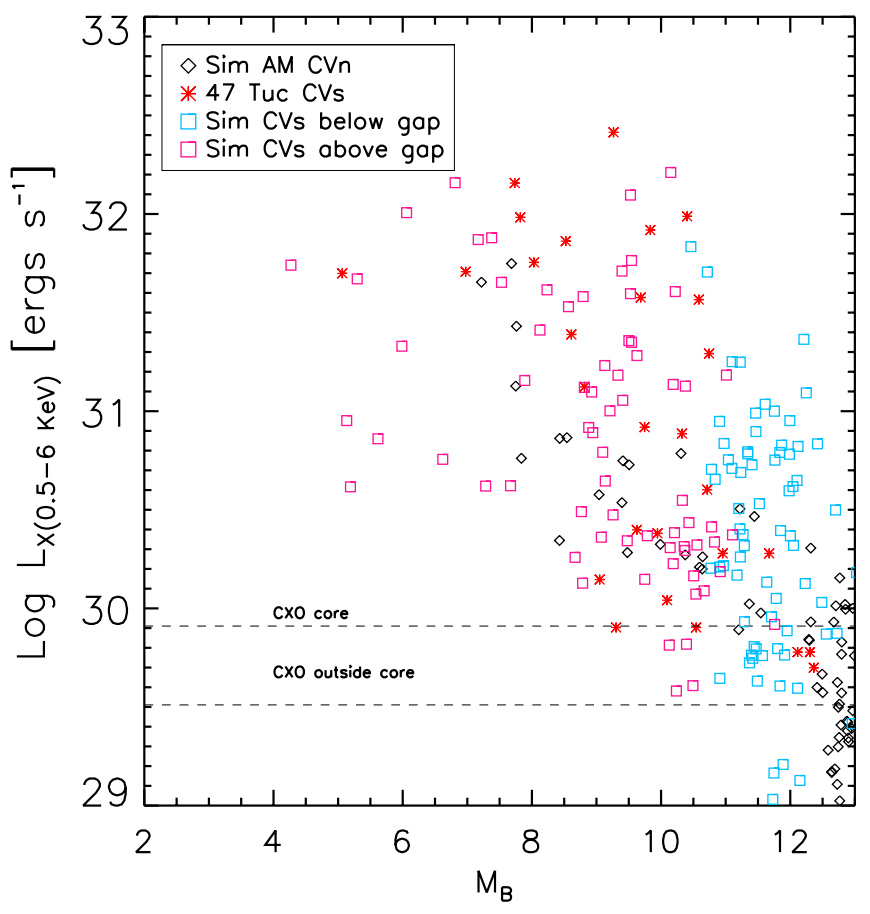

Figure 20. $M_{B}$ vs $\log L_{X}$ for CVs and AM CVn systems (sim CVs and sim AM CVn) obtained with the models of Ivanova et al. (2006). For comparison, we have plotted the CVs discussed in this paper, excluding those mentioned in Section 4.1.5 and the counterparts to W100, W120, W122 and W229. The dashed lines indicate the depth of the Chandra X-ray observations in 47 Tuc inside and outside the core.

relatively bright CVs in each cluster are a tracer of the density of the cluster core a couple of Gyrs ago. The relatively high number of bright CVs in NGC 6397 and NGC 6752 with respect to 47 Tuc, scaled by mass, indicates that these clusters have higher production rates of CVs per unit mass than 47 Tuc, as might be expected given their higher core densities (though NGC 6752's core density is only slightly higher). These clusters also have a relatively high number of bright CVs compared to 47 Tuc, when scaled by stellar encounter rate. This suggests that NGC 6397 and NGC 6752 have been at even higher densities at some time in the past couple Gyr, during which a large number of CVs with massive secondaries were generated, perhaps through episodes of deep core collapse (see e.g. Fregeau et al. 2003. Heggie \& Giersz 2009 Hurley \& Shara 2012). If so, we might expect that other dense clusters have been observed at times of maximum collapse, and show relatively few bright CVs for their stellar encounter rate. Alternatively, the difference could be due to other unmodeled dynamical or evolutionary effects. In contrast, the differences between the fainter CV populations are less strong, and in particular the faint $\mathrm{CV}$ populations in NGC 6752 and 47 Tuc are roughly comparable (when scaled by stellar encounter rate, or indeed by mass). The faint $\mathrm{CV}$ population likely reflects a combination of primordial CVs and CVs produced more than 1-2 Gyrs ago (as found in the models of Belloni et al. 2017b)

Using Monte Carlo simulations, Hong et al. (2017) studied the effect of stellar interactions on the $\mathrm{CV}$ population 
in GCs. They distinguished two groups of CVs, one group formed by primordial binaries $(\mathrm{P})$, which have experienced some changes in their orbital parameters but which keep the same initial components during the evolution of the cluster, and another group (E) in which the primordial binaries have suffered strong dynamical interactions leading to the replacement of one of the components. Group E has also more massive and younger companions than group $\mathrm{P}$. These authors found that the group $\mathrm{E}$ is radially more concentrated than the group $\mathrm{P}$. This is in agreement with findings for core-collapsed clusters like NGC 6397 and NGC 6752. However, in 47 Tuc we do not see such radial distribution (see Section 5.2). This suggests that there is not such a clear distinction between the older and younger CV populations in 47 Tuc as seen in the core-collapsed clusters, strengthening the case for a recent episode of close binary formation in the core-collapsed clusters.

We appear to see a higher ratio of older to younger CVs in 47 Tuc than in NGC 6752 and NGC 6397. This suggests that the core-collapse process may have formed many more young CVs in the last few Gyrs in NGC 6397 and NGC 6752. At the same time, that process may be more efficient in destroying ${ }^{6}$ and/or ejecting older CVs and chromospherically active binaries than creating them, as core-collapsed clusters seem to have a deficiency of X-ray binaries overall (Cohn et al. 2010, Bahramian et al. 2013), which would explain the bimodal CV luminosity distributions and different radial distributions of the CVs in core-collapsed clusters.

\section{SUMMARY}

We can summarize the findings of this paper as follows:

- In this work we report the identification of $43 \mathrm{CVs}$ and CV candidates in 47 Tuc using NUV and optical data from the HST. All of them are Chandra X-ray sources. Of these 43 systems, 22 are for the first time reported in this work. We found variability at a confidence level equal or higher than $95 \%$ for 9 out of these 22 objects. This suggests that these systems are indeed the real counterparts to the corresponding X-ray sources. With $43 \mathrm{CVs}$ and CV candidates, 47 Tuc is the GC with the most CVs identified so far.

- We have made use of the first deep $\mathrm{H} \alpha$ study of 47 Tuc with HST that uses near - simultaneous continuum - band photometry, which reduces the risk of obtaining misleading results since variability is accounted for.

- All the objects classified as CVs and CV candidates in this paper have a blue color in the NUV and in the optical CMDs due to emission from the accretor (the WD) and the accretion disk.

- For eighteen CVs out of the $43 \mathrm{CVs}$ and CV candidates in the cluster we have identified $\mathrm{H} \alpha$ emission likely coming from the accretion disk or stream, and contributions from the companion star. Unfortunately, because of stellar crowding, faintness, presence of saturated stars, objects outside of the optical FOV and bleeding trails, $\mathrm{H} \alpha$ photometry was not obtained for all the systems here reported.

6 Among the 'destruction' channels of CVs are unstable mass transfer, strong dynamical interactions and cluster escape (Belloni et al. 2017).
- We have identified four CVs that have large amplitude variations (up to $\sim 3$ magnitudes) in time-scales of hours in the filter $\mathrm{U}_{300}$. Flickering does not seem to be a plausible explanation for these variations. The dramatical changes of the eclipsing variable $\mathrm{W} 2_{U V}$ could be due to precession of the disk, or changes in the thickness of the accretion disk. Alternatively they could be due to low and high state switches, like happens in magnetic CVs.

- For the first time we compared the CV population in 47 Tuc (a non core collapsed cluster), to those of NGC 6397 and NGC 6752 (core-collapsed clusters).

- We found that unlike the luminosity functions in these two core-collapsed clusters, the NUV and optical luminosity functions of CVs in 47 Tuc are unimodal.

- 47 Tuc has many more faint CVs than those corecollapsed clusters. This suggests that the CV population in 47 Tuc is a combination of primordial CVs and dynamically $\mathrm{CVs}$ formed a long time ago

- If we scale the masses of NGC 6397 and NGC 6752 to that of 47 Tuc, the core-collapsed clusters have many more bright CVs. This seems to indicate that the dynamical interactions play a major role in core-collapsed clusters to create CVs.

- We showed that 47 Tuc and the core-collapsed clusters NGC 6397 and NGC 6752 have a bimodal distribution of $\mathrm{CVs}$ in X-rays. This could be related to the orbital periods of the systems and the donor mass transfer rates (the higher the mass transfer, the higher the X-ray luminosity). CVs below the period gap would have smaller transfer rates than CVs above the gap.

- The CVs in 47 Tuc are more concentrated in the core of the cluster than the MSTO stars. Given that faint and bright CVs are equally segregated, it means that they are more massive. In fact we found that CVs with $\mathrm{M}_{B 390} \leqslant 9$ could have $M_{\mathrm{WD}} \sim 0.9 M_{\odot}$ with a MS secondary of mass $\sim 0.55 M_{\odot}$. At the same time CVs with $\mathrm{M}_{B 390}>9$ could have secondary masses $\lesssim 0.2 M_{\odot}$ and $M_{\mathrm{WD}} \sim 1.2 M_{\odot}$. These massive WDs in faint CVs suggest a scenario in which there is a net increase in mass over the combined action of accretion and nova outbursts.

- In this paper we also present the first AM CVn candidate in 47 Tuc $\left(\mathrm{W} 62_{U V}\right)$. That object is blue in the NUV and optical. Its colors are consistent with those of confirmed AM CVns. It shows NUV variability at a confidence level higher than $99 \%$. It is also an X-ray source with $L_{X(0.5-6 \mathrm{keV})}=2.6 \times 10^{30} \mathrm{erg} \mathrm{s}^{-1}$ and it seems to have an $\operatorname{EW}(\mathrm{H} \alpha)$ emission line of $\sim-3 \pm 6 \AA$, consistent with zero. The $\mathrm{V}$ absolute magnitude of the system is consistent with that of an AM CVn with an orbital period of $\sim 6$ mins. All these characteristics would make it very similar to the known AM CVn system HM Cnc. If confirmed, it would be one of the few AM CVn systems with a period shorter than 10 mins. Also, It would help understanding the formation and evolution of AM CVn systems in dense stellar environments.

- The nature of $\mathrm{W} 21_{U V}$ is intriguing and uncertain since we have discarded possible explanations given its photometric behavior.

- Additionally, we discuss four counterparts to our X-ray sources that show NUV and optical colors similar to those found for MSP counterparts. The colors of three of these objects $\left(\mathrm{W} 70_{U V}, \mathrm{~W} 85_{U V}\right.$ and $\left.\mathrm{W} 253_{U V}\right)$ are consistent with 
having deep $\mathrm{H} \alpha$ absorption lines, which are compatible with those of DA WDs. The other system $\left(\mathrm{W} 34_{U V}\right)$ shows extremely strong heating (varying by $\sim 3$ mags in the NUV) which cannot be provided by the weak X-ray source, requiring irradiation by unobserved processes from an accretor star (e.g. particle heating from pulsar winds). The photometric results obtained for this object strongly suggest that this system could be an ablated (black widow or redback system) MSP companion. None of the four discussed objects have been reported as radio sources yet.

\section{ACKNOWLEDGMENTS}

The authors appreciate the comments by the anonymous referee, which improved this paper. LERS acknowledges support from NOVA and a CONACyT (Mexico) fellowship. COH acknowledges support from an NSERC Discovery Grant and a Discovery Accelerator Supplement. The authors would like to thank Dr. Andrew Dolphin who kindly answered questions about the usage of the DOLPHOT photometry package, as well to Dr. Paulo Freire for comments on the paper. 
Table 5: Results for the CVs and CV candidates in 47 Tuc.

\begin{tabular}{|c|c|c|c|c|c|c|c|c|c|c|c|c|}
\hline $\begin{array}{l}\text { W source } \\
\text { counterpart }\end{array}$ & $\begin{array}{l}\text { R.A. } \\
\text { (J2000) }\end{array}$ & $\begin{array}{c}\text { Decl. } \\
\text { (J2000) }\end{array}$ & $\mathrm{r}\left({ }^{\prime}\right)$ & $\begin{array}{c}d_{X} \\
\left(" / \sigma_{X}\right)\end{array}$ & $\mathrm{U}_{300}$ & $\mathrm{~B}_{390}$ & $\mathrm{~B}_{435}$ & $\mathrm{H} \alpha$ & ${ }_{\left(10^{30} \mathrm{erg} \mathrm{s}^{-1}\right)}^{\mathrm{R}_{625}}$ & $L_{X(0.5-6 \mathrm{keV})}$ & Type & Notes \\
\hline 1 & $00: 24: 16.969$ & $-72: 04: 27.20$ & 0.964 & $0.03 / 0.4$ & $23.04(1)$ & $23.94(2)$ & $20.25(1)$ & $19.31(1)$ & 19.99(1) & $97.3_{-2.2}^{+2.4}$ & $\mathrm{CV}$ & $\mathrm{p}-\mathrm{v}^{*}$ \\
\hline $2^{1}$ & $00: 24: 15.887$ & $-72: 04: 36.38$ & 0.828 & $0.009 / 0.1$ & $21.356(4)$ & $21.357(4)$ & $22.17(8)$ & $20.44(4)$ & $22.58(5)$ & $96.1_{-2.3}^{+2.4}$ & $\mathrm{CV}$ & $\mathrm{p}-\mathrm{v}$ \\
\hline 8 & $00: 24: 10.753$ & $-72: 04: 25.71$ & 0.594 & $0.03 / 0.3$ & $22.048(7)$ & $23.22(1)$ & $22.75(4)$ & $20.75(3)$ & $21.92(3)$ & $37.7_{-1.5}^{+1.6}$ & $\mathrm{CV}$ & $\mathrm{p}-\mathrm{v}$ \\
\hline $15^{1}$ & $00: 24: 08.479$ & $-72: 05: 00.31$ & 0.247 & $0.005 / 0.06$ & $21.666(6)$ & $22.147(8)$ & $22.77(6)$ & $20.78(4)$ & $21.73(4)$ & $24.5_{-1.3}^{+1.3}$ & $\mathrm{CV}$ & $\mathrm{p}-\mathrm{v}$ \\
\hline 16 & $00: 24: 08.300$ & $-72: 04: 35.79$ & 0.345 & $0.05 / 0.6$ & $21.97(2)$ & $22.35(2)$ & - & - & - & $13.3_{-0.9}^{+1.0}$ & $\mathrm{CV}$ & $\mathrm{C}-\mathrm{V}$ \\
\hline $23^{1}$ & $00: 24: 07.804$ & $-72: 04: 41.53$ & 0.246 & $0.03 / 0.4$ & $23.03(2)$ & $22.45(2)$ & $22.27(2)$ & $20.11(2)$ & $20.47(1)$ & $\leqslant 41.8_{-1.6}^{+1.7}$ & $\mathrm{CV}$ & $\mathrm{n}-\mathrm{v}$ \\
\hline 24 & $00: 24: 07.380$ & $-72: 04: 49.65$ & 0.138 & $0.06 / 0.7$ & $22.94(1)$ & $24.32(4)$ & $24.36(8)$ & - & - & $\leqslant 31.8_{-1.6}^{+1.7}$ & CV? & $\mathrm{n}$ \\
\hline 25 & $00: 24: 07.142$ & $-72: 05: 45.75$ & 0.891 & $0.03 / 0.4$ & $20.702(8)$ & $21.568(7)$ & $20.44(3)$ & $18.89(2)$ & $19.62(3)$ & $56.8_{-1.7}^{+1.9^{\circ}}$ & $\mathrm{CV}$ & $\mathrm{p}-\mathrm{v}$ \\
\hline $27^{1}$ & $00: 24: 06.373$ & $-72: 04: 43.00$ & 0.169 & $0.02 / 0.3$ & $20.943(4)$ & $22.80(1)$ & $21.99(2)$ & $20.20(3)$ & $21.01(5)$ & $259.3_{-4.1}^{+4.2}$ & $\mathrm{CV}$ & $\mathrm{p}-\mathrm{v}$ \\
\hline 30 & $00: 24: 06.002$ & $-72: 04: 56.16$ & 0.061 & $0.03 / 0.4$ & $19.74(4)$ & $20.51(1)$ & $21.69(1)$ & $20.10(3)$ & $20.62(2)$ & $51.0_{-1.7}^{+1.8}$ & $\mathrm{CV}$ & $\mathrm{p}-\mathrm{v}$ \\
\hline 33 & $00: 24: 05.381$ & $-72: 04: 21.67$ & 0.517 & $0.15 / 1.6$ & $22.392(8)$ & $23.28(1)$ & $23.33(6)$ & $21.24(3)$ & $22.21(4)$ & $8.3_{-0.7}^{+0.8}$ & $\mathrm{CV}$ & $\mathrm{p}-\mathrm{v}$ \\
\hline 35 & $00: 24: 05.024$ & $-72: 05: 06.30$ & 0.230 & $0.4 / 2.5$ & $22.59(3)$ & $21.87(3)$ & $20.96(5)$ & - & - & $\leqslant 1.0_{-0.2}^{+0.4}$ & $\mathrm{CV}$ & $\mathrm{p}-\mathrm{v}$ \\
\hline 36 & $00: 24: 04.924$ & $-72: 04: 55.45$ & 0.075 & $0.09 / 1.1$ & $18.97(3)$ & $18.6(2)$ & $18.12(1)$ & $16.66(1)$ & $16.98(1)$ & $50.0_{-2.2}^{+2.2}$ & $\mathrm{CV}$ & $\mathrm{p}-\mathrm{v}$ \\
\hline 44 & $00: 24: 03.688$ & $-72: 04: 58.98$ & 0.187 & $0.08 / 1.0$ & $22.98(3)$ & $24.28(6)$ & - & - & - & $19.6_{-1.1}^{+1.2}$ & $\mathrm{CV}$ & $\mathrm{p}-\mathrm{v}^{*}$ \\
\hline 45 & $00: 24: 03.764$ & $-72: 04: 22.97$ & 0.517 & $0.03 / 0.3$ & $20.83(2)$ & $22.06(2)$ & $22.06(3)$ & $20.29(2)$ & $21.39(2)$ & $72.8_{-2.0}^{+2.1}$ & $\mathrm{CV}$ & $\mathrm{p}-\mathrm{v}$ \\
\hline 49 & $00: 24: 03.102$ & $-72: 04: 47.34$ & 0.219 & $0.07 / 0.2$ & $23.54(2)$ & $24.25(3)$ & $25.5(2)$ & - & - & $4.0_{-0.6}^{+0.8}$ & $\mathrm{CV}$ & $\mathrm{p}-\mathrm{v}$ \\
\hline 51 & $00: 24: 02.814$ & $-72: 04: 49.10$ & 0.232 & $0.1 / 1.6$ & $23.81(3)$ & $23.37(2)$ & $23.13(7)$ & $20.65(5)$ & $21.41(6)$ & $82.9_{-2.2}^{+0.6}$ & $\mathrm{CV}$ & $\mathrm{p}-\mathrm{v}$ \\
\hline 53 & $00: 24: 02.533$ & $-72: 05: 11.26$ & 0.394 & $0.03 / 0.3$ & $24.49(3)$ & $24.12(2)$ & $23.52(4)$ & $20.93(2)$ & - & $36.8_{-1.4}^{+1.5}$ & $\mathrm{CV}$ & $\mathrm{p}-\mathrm{v}^{*}$ \\
\hline 55 & $00: 24: 02.185$ & $-72: 04: 50.28$ & 0.273 & $0.3 / 2.5$ & $22.24(2)$ & $23.48(3)$ & $24.04(6)$ & - & - & $2.4_{-0.4}^{+0.5}$ & CV? & $\mathrm{c}$ \\
\hline 56 & $00: 24: 02.125$ & $-72: 05: 42.02$ & 0.866 & $0.01 / 0.1$ & $20.33(2)$ & $21.27(1)$ & $21.41(2)$ & $19.55(3)$ & $20.56(1)$ & $143.5_{-2.8}^{+2.9}$ & $\mathrm{CV}$ & $\mathrm{p}-\mathrm{v}$ \\
\hline 75 & $00: 24: 06.425$ & $-72: 04: 52.35$ & 0.055 & $0.3 / 3.7$ & $21.391(5)$ & $23.09(2)$ & $23.54(9)$ & - & - & $\leqslant 5.6_{-0.6}^{+0.8}$ & CV? & $\mathrm{n}$ \\
\hline 98 & $00: 24: 05.108$ & $-72: 05: 02.92$ & 0.176 & $0.1 / 1.3$ & $22.75(1)$ & $23.16(2)$ & - & - & - & $2.5_{-0.4}^{+0.5}$ & CV? & $\mathrm{n}$ \\
\hline $100-\mathrm{a}^{2}$ & $00: 24: 01.318$ & $-72: 04: 26.99$ & 0.545 & $0.25 / 1.5$ & $24.62(3)$ & $25.29(4)$ & $26.3(2)$ & - & - & $1.7_{-0.3}^{+0.4}$ & CV? & $\mathrm{n}$ \\
\hline $100-b^{2}$ & $00: 24: 01.281$ & $-72: 04: 27.11$ & 0.545 & $0.18 / 1.0$ & $25.76(7)$ & $25.56(6)$ & - & - & - & & $\mathrm{CV} ?$ & $\mathrm{n}$ \\
\hline 120 & $00: 24: 11.097$ & $-72: 06: 20.03$ & 1.514 & - & - & - & $23.40(1)$ & $22.67(3)$ & $23.07(2)$ & $15.0_{-10}^{+1.1}$ & $\mathrm{CV}$ & $\mathrm{p}$ \\
\hline 122 & $00: 24: 03.849$ & $-72: 06: 21.60$ & 1.488 & - & - & - & $23.25(2)$ & $21.48(1)$ & $22.36(1)$ & $20.2_{-1.1}^{+1.0}$ & $\mathrm{CV}$ & $\mathrm{p}$ \\
\hline 140 & $00: 24: 09.213$ & $-72: 05: 43.87$ & 0.894 & $0.04 / 0.4$ & $22.62(1)$ & $23.86(2)$ & $24.4(1)$ & - & $24.2(3)$ & $7.7_{-0.7}^{+0.8}$ & $\mathrm{CV}$ & $\mathrm{c}-\mathrm{V}$ \\
\hline 187 & $00: 23: 56.897$ & $-72: 03: 41.33$ & 1.369 & $0.3 / 2.2$ & $24.09(1)$ & $25.21(3)$ & - & - & - & $1.9_{-0.3}^{+0.5}$ & CV? & $\mathrm{n}$ \\
\hline 200 & $00: 24: 07.125$ & $-72: 05: 06.81$ & 0.259 & $0.2 / 2.0$ & $22.226(8)$ & $23.78(3)$ & $23.37(5)$ & - & $24.35(2)$ & $\leqslant 4.1_{-0.5}^{+0.6}$ & $\mathrm{CV}$ & $\mathrm{n}-\mathrm{v} ?$ \\
\hline 202 & $00: 24: 04.946$ & $-72: 04: 43.71$ & 0.161 & $0.3 / 2.7$ & $21.472(5)$ & $23.11(4)$ & $23.16(5)$ & - & - & $\leqslant 3.6_{-0.5}^{+0.6}$ & CV? & $\mathrm{n}$ \\
\hline 223 & $00: 24: 20.916$ & $-72: 05: 18.62$ & 1.246 & $0.3 / 1.5$ & $24.63(3)$ & $25.84(7)$ & - & - & - & $0.6_{-0.2}^{+0.3}$ & CV? & $\mathrm{n}$ \\
\hline 229 & $00: 24: 18.511$ & $-72: 03: 34.79$ & 1.629 & $0.1 / 0.8$ & $25.6(2)$ & $<26.5$ & - & - & - & $1.2_{-0.3}^{+0.4}$ & CV? & $\mathrm{n}$ \\
\hline 235 & $00: 24: 15.986$ & $-72: 04: 12.52$ & 1.036 & $0.3 / 1.4$ & $24.59(3)$ & $25.65(4)$ & $25.60(8)$ & - & - & $0.6_{-0.2}^{+0.3}$ & CV? & $\mathrm{n}$ \\
\hline 256 & $00: 24: 10.047$ & $-72: 04: 26.64$ & 0.547 & $0.6 / 1.7$ & $24.20(2)$ & $25.23(5)$ & $25.6(1)$ & - & - & $\leqslant 0.7_{-0.2}^{+0.4}$ & $\mathrm{CV}$ & $\mathrm{n}-\mathrm{v} ?$ \\
\hline 292 & $00: 24: 04.972$ & $-72: 03: 30.14$ & 1.377 & $0.3 / 1.7$ & $23.55(2)$ & $24.49(2)$ & - & - & - & $1.9_{-0.3}^{+0.5}$ & $\mathrm{CV}$ & $n-v$ \\
\hline 295 & $00: 24: 04.657$ & $-72: 05: 09.46$ & 0.291 & $0.09 / 0.6$ & $22.56(1)$ & $23.63(3)$ & $23.7(1)$ & $21.5(1)$ & $21.90(7)$ & $1.1_{-0.3}^{+0.5}$ & $\mathrm{CV}$ & $\mathrm{n}-\mathrm{v}$ \\
\hline 299 & $00: 24: 04.136$ & $-72: 05: 12.32$ & 0.348 & $0.1 / 1.2$ & $23.72(6)$ & $24.3(1)$ & $23.7(1)$ & $21.59(8)$ & $22.06(9)$ & $\leqslant 1.8_{-0.3}^{+0.5}$ & $\mathrm{CV}$ & $\mathrm{n}-\mathrm{v} ?$ \\
\hline
\end{tabular}




\begin{tabular}{|c|c|c|c|c|c|c|c|c|c|c|c|c|}
\hline $\begin{array}{c}\text { W source } \\
\text { counterpart }\end{array}$ & $\begin{array}{l}\text { R.A. } \\
\text { (J2000) }\end{array}$ & $\begin{array}{c}\text { Decl. } \\
(\mathrm{J} 2000)\end{array}$ & $r\left({ }^{\prime}\right)$ & $\begin{array}{c}d_{X} \\
\left({ }^{\prime \prime} / \sigma_{X}\right) \\
\end{array}$ & $\mathrm{U}_{300}$ & $\begin{array}{c}\text { Continued } \\
\mathrm{B}_{390}\end{array}$ & $\mathrm{~B}_{435}$ & $\mathrm{H} \alpha$ & $\mathrm{R}_{625}$ & $\begin{array}{l}L_{X(0.5-6 \mathrm{keV})} \\
\left(10^{30} \mathrm{erg} \mathrm{s}^{-1}\right)\end{array}$ & Type & Notes \\
\hline 302 & $00: 24: 02.976$ & $-72: 04: 43.80$ & 0.258 & $0.3 / 0.8$ & $23.07(1)$ & $24.38(3)$ & $24.7(1)$ & - & - & $\leqslant 1.2_{-0.3}^{+0.4}$ & CV? & $\mathrm{n}$ \\
\hline 304 & $00: 24: 02.047$ & $-72: 05: 01.75$ & 0.319 & $0.4 / 2.4$ & 23.11(1) & $23.83(2)$ & $25.3(3)$ & - & - & $\leqslant 0.9_{-0.2}^{+0.4}$ & $\mathrm{CV}$ & $\mathrm{n}-\mathrm{v} ?$ \\
\hline 309 & $00: 24: 01.070$ & $-72: 05: 30.48$ & 0.724 & $0.3 / 1.9$ & $23.32(1)$ & $22.585(7)$ & - & - & - & $1.4_{-0.3}^{+0.4}$ & $\mathrm{CV}$ & $n-v$ \\
\hline 311 & $00: 24: 00.631$ & $-72: 04: 12.72$ & 0.772 & $0.3 / 1.7$ & $23.29(2)$ & $24.08(9)$ & $24.9(1)$ & - & - & $0.8_{-0.2}^{+0.5}$ & $\mathrm{CV}$ & $n-v$ \\
\hline 324 & 00:23:57.691 & $-72: 04: 14.27$ & 0.889 & $0.2 / 1.1$ & $21.882(6)$ & $22.84(1)$ & - & - & - & $0.8_{-0.2}^{+0.4}$ & CV? & $\mathrm{n}$ \\
\hline 329 & $00: 23: 55.025$ & $-72: 04: 34.22$ & 0.878 & $0.5 / 2.2$ & $25.02(6)$ & $25.9(1)$ & - & - & - & $0.5_{-0.2}^{+0.3}$ & CV? & $\mathrm{n}$ \\
\hline 335 & $00: 23: 52.053$ & $-72: 04: 37.11$ & 1.082 & $0.4 / 2.4$ & $24.30(2)$ & $24.94(3)$ & $25.8(2)$ & - & - & $\leqslant 1.1_{-0.2}^{+0.4}$ & CV? & $\mathrm{n}$ \\
\hline 21 & $00: 24: 07.766$ & $-72: 05: 27.28$ & 0.597 & $0.06 / 0.7$ & $21.381(7)$ & $21.43(1)$ & $21.17(1)$ & $20.29(2)$ & $20.57(2)$ & $14.2_{-0.9}^{+1.0}$ & $?$ & $r-v$ \\
\hline 62 & $00: 23: 59.340$ & $-72: 04: 48.25$ & 0.049 & $0.1 / 1.0$ & $19.651(2)$ & $19.71(3)$ & $19.54(2)$ & $19.04(5)$ & $19.16(6)$ & $2.6_{-0.4}^{+0.5}$ & AM CVn? & $n-v$ \\
\hline
\end{tabular}

NOTES.- Astrometric positions correspond to the counterparts associated to the respective W source. Units of right ascension are hours, minutes, and seconds, and

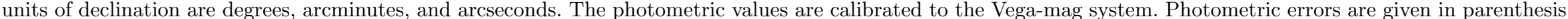

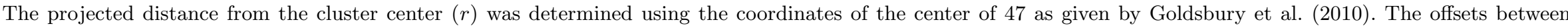

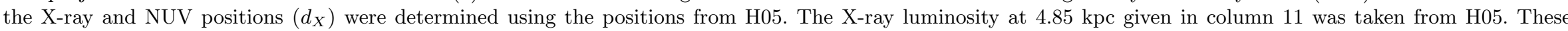

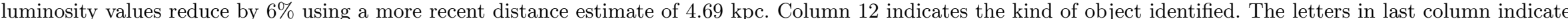

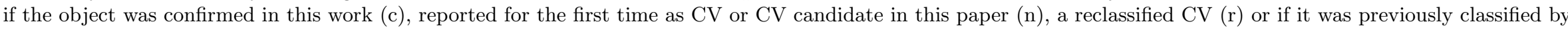

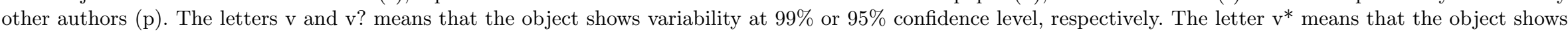
variability in the I or F300W band according to E03b.

1 Mean magnitudes.

${ }^{2}$ For this object the correct counterpart is ambiguous. 


\section{REFERENCES}

Albrow M. D., Gilliland R. L., Brown T. M., Edmonds P. D., Guhathakurta P., Sarajedini A., 2001, ApJ, 559, 1060

Augusteijn T., van der Hooft F., de Jong J. A., van Paradijs J., 1996, A\&A, 311, 889

Auriere M., Koch-Miramond L., Ortolani S., 1989, A\&A, 214,113

Bahramian A., Heinke C. O., Sivakoff G. R., Gladstone J. C., 2013, ApJ, 766, 136

Baraffe I., Chabrier G., Allard F., Hauschildt P. H., 1997, A\&A, 327, 1054

Bassa C. G., van Kerkwijk M. H., Koester D., Verbunt F., 2006, A\&A, 456, 295

Beccari G., De Marchi G., Panagia N., Pasquini L., 2014, MNRAS, 437, 2621

Belloni D., Giersz M., Askar A., Leigh N., Hypki A., 2016, MNRAS, 462, 2950

Belloni D., Giersz M., Rocha-Pinto H. J., Leigh N. W. C., Askar A., 2017, MNRAS, 464, 4077

Belloni D., Zorotovic M., Schreiber M. R., Leigh N. W. C., Giersz M., Askar A., 2017, MNRAS, 468, 2429

Bildsten L., Townsley D. M., Deloye C. J., Nelemans G., 2006, ApJ, 640, 466

Bogdanov S., Grindlay J. E., Heinke C. O., Camilo F., Freire P. C. C., Becker W., 2006, ApJ, 646, 1104

Bogdanov S., van den Berg M., Heinke C. O., Cohn H. N., Lugger P. M., Grindlay J. E., 2010, ApJ, 709, 241

Bressan A., Marigo P., Girardi L., Salasnich B., Dal Cero C., Rubele S., Nanni A., 2012, MNRAS, 427, 127

Cadelano M., Pallanca C., Ferraro F. R., Stairs I., Ransom S. M., Dalessandro E., Lanzoni B., Hessels J. W. T., Freire P. C. C., 2015, ApJ, 807, 91

Carter P. J., Marsh T. R., Steeghs D., Groot P. J., Nelemans G., Levitan D., Rau A., Copperwheat C. M., Kupfer T., Roelofs G. H. A., 2013, MNRAS, 429, 2143

Cohn H. N., Lugger P. M., Couch S. M., Anderson J., Cool A. M., van den Berg M., Bogdanov S., Heinke C. O., Grindlay J. E., 2010, ApJ, 722, 20

Cool A. M., Haggard D., Arias T., Brochmann M., Dorfman J., Gafford A., White V., Anderson J., 2013, ApJ, 763,126

Davies M. B., 1997, MNRAS, 288, 117

Di Stefano R., Rappaport S., 1994, ApJ, 423, 274

Dieball A., Long K. S., Knigge C., Thomson G. S., Zurek D. R., 2010, ApJ, 710, 332

Dolphin A. E., 2000, PASP, 112, 1383

Edmonds P. D., Gilliland R. L., Camilo F., Heinke C. O., Grindlay J. E., 2002, ApJ, 579, 741

Edmonds P. D., Gilliland R. L., Heinke C. O., Grindlay J. E., 2003a, ApJ, 596, 1177 (E03a)

Edmonds P. D., Gilliland R. L., Heinke C. O., Grindlay J. E., 2003b, ApJ, 596, 1197 (E03b)

Ferraro F. R., Lapenna E., Mucciarelli A., Lanzoni B., Dalessandro E., Pallanca C., Massari D., 2016, ApJ, 816, 70 Forestell L. M., Heinke C. O., Cohn H. N., Lugger P. M., Sivakoff G. R., Bogdanov S., Cool A. M., Anderson J., 2014, MNRAS, 441, 757

Fregeau J. M., Gürkan M. A., Joshi K. J., Rasio F. A., 2003, ApJ, 593, 772

Gänsicke B. T., Dillon M., Southworth J., Thorstensen
J. R., Rodríguez-Gil P., Aungwerojwit A., Marsh T. R., Szkody P., Barros S. C. C., Casares J., de Martino D., Groot P. J., Hakala P., Kolb U., Littlefair S. P., et al. 2009, MNRAS, 397, 2170

Goldsbury R., Richer H. B., Anderson J., Dotter A., Sarajedini A., Woodley K., 2010, AJ, 140, 1830

Grindlay J. E., Cool A. M., Callanan P. J., Bailyn C. D., Cohn H. N., Lugger P. M., 1995, ApJ, 455, L47

Grindlay J. E., Heinke C., Edmonds P., Murray S. S., 2001, Science, 292, 2290

Grindlay J. E., Heinke C. O., Edmonds P. D., Murray S. S., Cool A. M., 2001, ApJ, 563, L53

Harlaftis E. T., Horne K., 1999, MNRAS, 305, 437

Harris W. E., 1996, AJ, 112, 1487

Heggie D. C., Giersz M., 2009, MNRAS, 397, L46

Heinke C. O., Grindlay J. E., Edmonds P. D., Cohn H. N., Lugger P. M., Camilo F., Bogdanov S., Freire P. C., 2005, ApJ, 625, 796 (H05)

Heyl J. S., Richer H., Anderson J., Fahlman G., Dotter A., Hurley J., Kalirai J., Rich R. M., Shara M., Stetson P., Woodley K. A., Zurek D., 2012, ApJ, 761, 51

Hong J., Vesperini E., Belloni D., Giersz M., 2017, MNRAS, 464, 2511

Howell J. H., Guhathakurta P., Gilliland R. L., 2000, PASP, 112,1200

Howell S. B., Nelson L. A., Rappaport S., 2001, ApJ, 550, 897

Huang R. H. H., Becker W., Edmonds P. D., Elsner R. F., Heinke C. O., Hsieh B. C., 2010, A\&A, 513, A16

Hurley J. R., Shara M. M., 2012, MNRAS, 425, 2872

Israel G. L., Esposito P., Rodríguez Castillo G. A., Sidoli L., 2016, MNRAS, 462, 4371

Israel G. L., Hummel W., Covino S., Campana S., Appenzeller I., Gässler W., Mantel K.-H., Marconi G., Mauche C. W., Munari U., Negueruela I., Nicklas H., Rupprecht G., Smart R. L., Stahl O., Stella L., 2002, A\&A, 386, L13 Ivanova N., Heinke C. O., Rasio F. A., Taam R. E., Belczynski K., Fregeau J., 2006, MNRAS, 372, 1043

Kimmig B., Seth A., Ivans I. I., Strader J., Caldwell N., Anderton T., Gregersen D., 2015, AJ, 149, 53

King I. R., 1966, AJ, 71, 64

Knigge C., Baraffe I., Patterson J., 2011, ApJS, 194, 28

Knigge C., Dieball A., Maíz Apellániz J., Long K. S., Zurek D. R., Shara M. M., 2008, ApJ, 683, 1006

Knigge C., Zurek D. R., Shara M. M., Long K. S., 2002, ApJ, 579, 752

Knigge C., Zurek D. R., Shara M. M., Long K. S., Gilliland R. L., 2003, ApJ, 599, 1320

Kuiper N. H., 1960, Proc. of the Koninkl. Nederl. Akad. van Wetenschappen, Ser. A., 63, 38

Lu T.-N., Kong A. K. H., Verbunt F., Lewin W. H. G., Anderson S. F., Pooley D., 2011, ApJ, 736, 158

Lugger P. M., Cohn H. N., Cool A. M., Heinke C. O., Anderson J., 2017, ApJ, 841, 53

Lugger P. M., Cohn H. N., Grindlay J. E., 1995, ApJ, 439, 191

Lugger P. M., Cohn H. N., Heinke C. O., Grindlay J. E., Edmonds P. D., 2007, ApJ, 657, 286

Majewski S. R., Patterson R. J., Dinescu D. I., Johnson W. Y., Ostheimer J. C., Kunkel W. E., Palma C., 2000, in Noels A., Magain P., Caro D., Jehin E., Parmentier G., Thoul A. A., eds, Liege International Astrophysical 
Colloquia Vol. 35 of Liege International Astrophysical Colloquia, $\omega$ Centauri : Nucleus of a milky way dwarf spheroidal?. p. 619

Maxwell J. E., Lugger P. M., Cohn H. N., Heinke C. O., Grindlay J. E., Budac S. A., Drukier G. A., Bailyn C. D., 2012, ApJ, 756, 147

Miller-Jones J. C. A., Strader J., Heinke C. O., Maccarone T. J., van den Berg M., Knigge C., Chomiuk L., Noyola E., Russell T. D., Seth A. C., Sivakoff G. R., 2015, MNRAS, 453, 3918

Mukai K., 2017, PASP, 129, 062001

Nelemans G., 2005, in Hameury J.-M., Lasota J.-P., eds, The Astrophysics of Cataclysmic Variables and Related Objects Vol. 330 of Astronomical Society of the Pacific Conference Series, AM CVn stars. p. 27

Nelson L. A., Rappaport S. A., Joss P. C., 1986, ApJ, 304, 231

Nice D. J., Thorsett S. E., 1992, ApJ, 397, 249

Noyola E., Gebhardt K., Bergmann M., 2008, ApJ, 676, 1008

Pan Z., Hobbs G., Li D., Ridolfi A., Wang P., Freire P., 2016, MNRAS, 459, L26

Paresce F., de Marchi G., 1994, ApJ, 427, L33

Paresce F., de Marchi G., Ferraro F. R., 1992, Nature, 360, 46

Parzen E., 1962, Ann. Math. Statist., 33, 1065

Pooley D., Lewin W. H. G., Homer L., Verbunt F., Anderson S. F., Gaensler B. M., Margon B., Miller J. M., Fox D. W., Kaspi V. M., van der Klis M., 2002, ApJ, 569, 405 Pretorius M. L., Knigge C., Schwope A. D., 2013, MNRAS, 432, 570

Ramsay G., Groot P. J., Marsh T., Nelemans G., Steeghs D., Hakala P., 2006, A\&A, 457, 623

Reynolds M. T., Callanan P. J., Fruchter A. S., Torres M. A. P., Beer M. E., Gibbons R. A., 2007, MNRAS, 379,1117

Rivera Sandoval L. E., Hernandez Santisteban J. V., Degenaar N., Wijnands R., Knigge C., Miller J. M., Reynolds M., Altamirano D., van den Berg M., Hill A., 2017, ArXiv e-prints

Rivera-Sandoval L. E., van den Berg M., Heinke C. O., Cohn H. N., Lugger P. M., Freire P., Anderson J., Serenelli A. M., Althaus L. G., Cool A. M., Grindlay J. E., Edmonds P. D., Wijnands R., Ivanova N., 2015, MNRAS, 453, 2707

Rodríguez-Gil P., Gänsicke B. T., Hagen H.-J., Nogami D., Torres M. A. P., Lehto H., Aungwerojwit A., Littlefair S., Araujo-Betancor S., Engels D., 2005, A\&A, 440, 701

Romani R. W., Filippenko A. V., Cenko S. B., 2014, ApJ, 793, L20

Salaris M., Held E. V., Ortolani S., Gullieuszik M., Momany Y., 2007, A\&A, 476, 243

Serenelli A. M., Althaus L. G., Rohrmann R., Benvenuto O. G., 2002, MNRAS, 337, 1091

Servillat M., Dieball A., Webb N. A., Knigge C., Cornelisse R., Barret D., Long K. S., Shara M. M., Zurek D. R., 2008, A\&A, 490, 641

Shara M. M., Bergeron L. E., Gilliland R. L., Saha A., Petro L., 1996, ApJ, 471, 804

Shara M. M., Hurley J. R., 2006, ApJ, 646, 464

Silverman B. W., 1986, Density estimation for statistics and data analysis
Sirianni M., Jee M. J., Benítez N., Blakeslee J. P., Martel A. R., Meurer G., Clampin M., De Marchi G., Ford H. C., Gilliland R., Hartig G. F., Illingworth G. D., Mack J., McCann W. J., 2005, PASP, 117, 1049

Solheim J.-E., 2010, PASP, 122, 1133

Steeghs D., Knigge C., Drew J., Unruh Y., Greimel R., 2008, The Astronomer's Telegram, 1653

Szkody P., Anderson S. F., Brooks K., Gänsicke B. T., Kronberg M., Riecken T., Ross N. P., Schmidt G. D., Schneider D. P., Agüeros M. A., Gomez-Moran A. N., Knapp G. R., Schreiber M. R., Schwope A. D., 2011, AJ, 142, 181

Thomson G. S., Knigge C., Dieball A., Maccarone T. J., Dolphin A., Zurek D., Long K. S., Shara M., Sarajedini A., 2012, MNRAS, 423, 2901

Thorstensen J. R., Fenton W. H., Patterson J. O., Kemp J., Krajci T., Baraffe I., 2002, ApJ, 567, L49

Trager S. C., King I. R., Djorgovski S., 1995, AJ, 109, 218 van den Berg M., Hong J. S., Grindlay J. E., 2009, ApJ, 700, 1702

van Kerkwijk M. H., Bergeron P., Kulkarni S. R., 1996, ApJ, 467, L89

VandenBerg D. A., Brogaard K., Leaman R., Casagrande L., 2013, ApJ, 775, 134

Verbunt F., Meylan G., 1988, A\&A, 203, 297

Warner B., 1987, MNRAS, 227, 23

Wijnen T. P. G., Zorotovic M., Schreiber M. R., 2015, A\&A, 577, A143

Witham A. R., Knigge C., Gänsicke B. T., Aungwerojwit A., Corradi R. L. M., Drew J. E., Greimel R., Groot P. J., Morales-Rueda L., Rodriguez-Flores E. R., Rodriguez-Gil P., Steeghs D., 2006, MNRAS, 369, 581

Woodley K. A., Goldsbury R., Kalirai J. S., Richer H. B., Tremblay P.-E., Anderson J., Bergeron P., Dotter A., Esteves L., Fahlman G. G., Hansen B. M. S., Heyl J., Hurley J., Rich R. M., Shara M. M., Stetson P. B., 2012, AJ, 143, 50

Zamanov R. K., Latev G. Y., Stoyanov K. A., Boeva S., Spassov B., Tsvetkova S. V., 2012, Astronomische Nachrichten, 333, 736

Zorotovic M., Schreiber M. R., Gänsicke B. T., 2011, A\&A, 536, A42

Zurek D. R., Knigge C., Maccarone T. J., Pooley D., Dieball A., Long K. S., Shara M., Sarajedini A., 2016, MNRAS, 460, 3660 


\section{APPENDIX A: THE CORE OF 47 TUC IN NUV}

We show a stacked image in the filter $\mathrm{U}_{300}$ which shows the portion of 47 Tuc covered in our study.

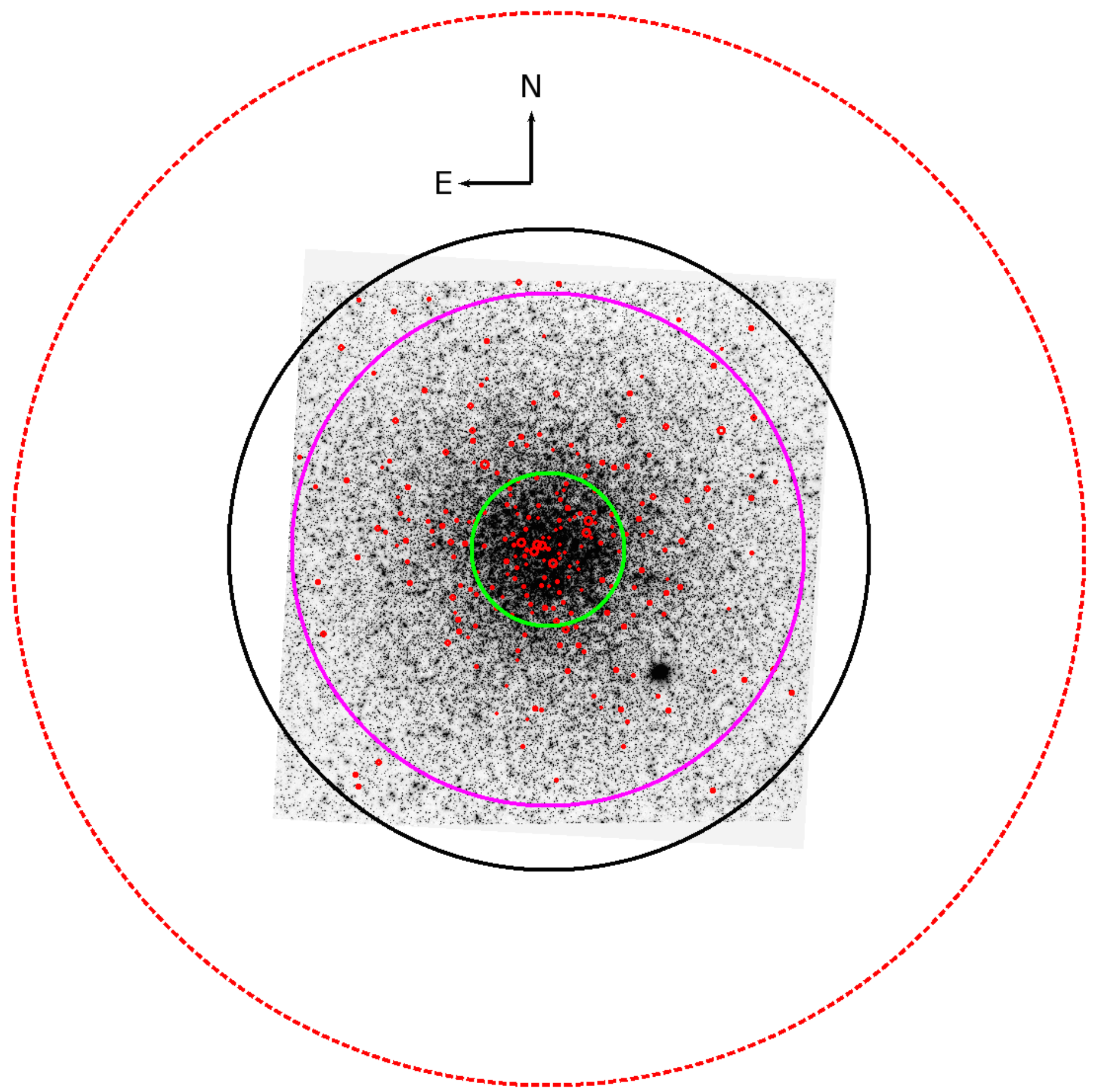

Figure A1. Stacked HST $\mathrm{U}_{300}$ image of 47 Tuc with $3 \sigma$ Chandra source error circles (small red circles). The red dashed circle indicates the $\mathrm{r}_{h}$ of the cluster $(2 ! 79)$. The green circle shows the core radius $\left(24^{\prime \prime}\right)$. The black circle has a radius of $100^{\prime \prime}$, and the magenta one indicates the largest circle that can be inscribed in our NUV images $\left(80^{\prime \prime}\right)$. There are $88 \mathrm{X}$-ray sources within the core radius out of the 238 sources detected in our FOV. 


\section{APPENDIX B: FINDING CHARTS}

We present finding charts in the NUV for some CVs and CV candidates discussed in section 4 For more finding charts see E03a, E03b, Knigge et al. (2008) and Beccari et al. (2014).
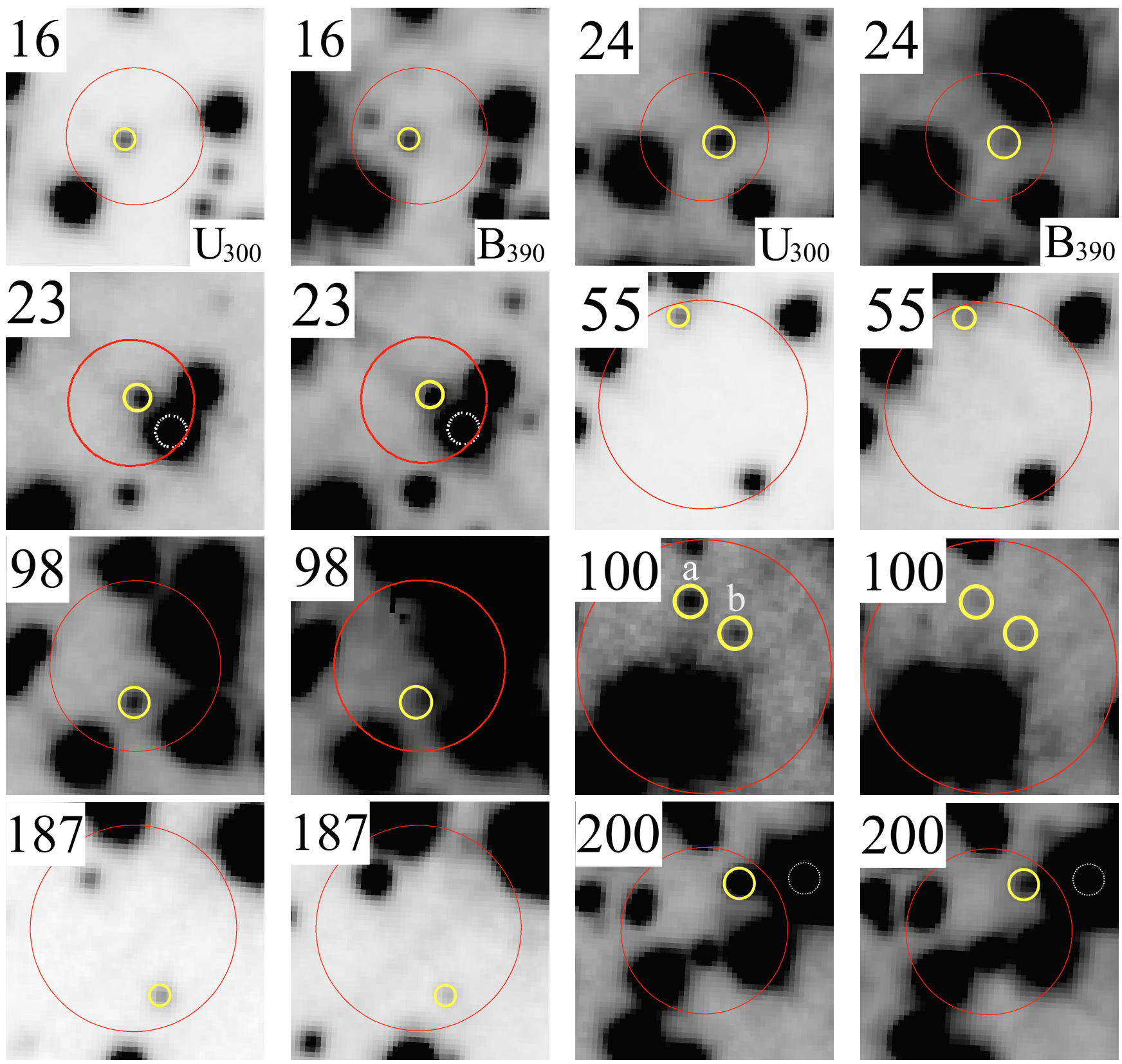

Figure B1. Finding charts in the filters $\mathrm{U}_{300}$ and $\mathrm{B}_{390}$ for some CVs and CV candidates in 47 Tuc. The solid red circles are the $3 \sigma$ match circles of Chandra sources. The CVs and CV candidates are indicated with a solid yellow circle. The dashed white circles indicate possible additional X-ray counterparts (see section 4.1.5). Each image is $1^{\prime \prime} \times 1^{\prime \prime}$. In each column the images correspond to the same filter that is indicated by the top image of the column. The pixel scale is $0{ }^{\prime \prime} 02$. 

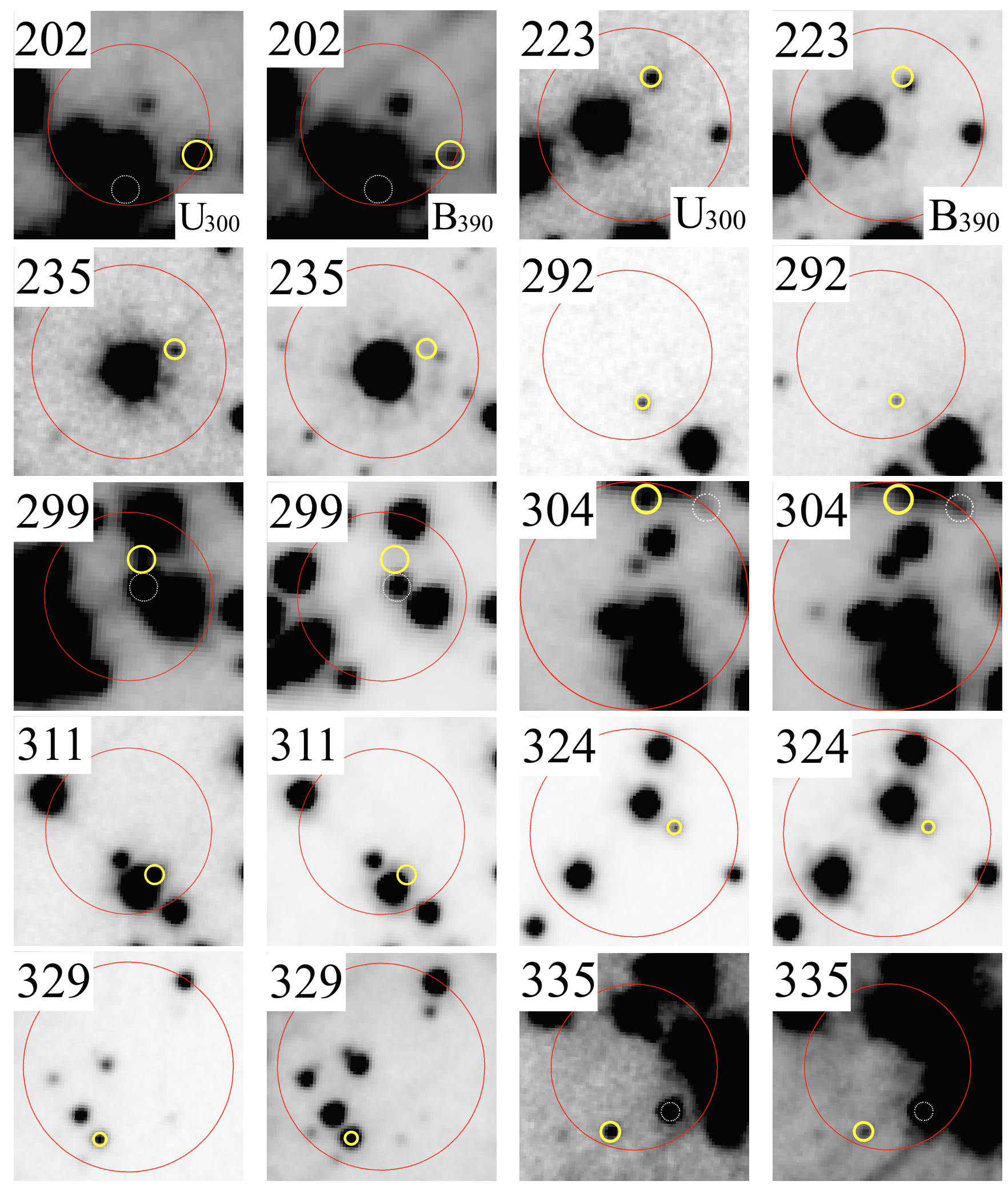

Figure B2. Finding charts in the filters $\mathrm{U}_{300}$ and $\mathrm{B}_{390}$ for some CVs and CV candidates in 47 Tuc. The solid red circles are the $3 \sigma$ match circles of Chandra sources. The CVs and CV candidates are indicated with a solid yellow circle. The dashed white circles indicate possible additional X-ray counterparts (see section 4.1.5). Each image is $1.5^{\prime \prime} \times 1.5^{\prime \prime}$, except for W202, W299 and W304 which images are $1^{\prime \prime} \times 1^{\prime \prime}$. In each column the images correspond to the same filter that is indicated by the top image of the column. The pixel scale is $0 . \prime 02$. 


\section{APPENDIX C: CATACLYSMIC VARIABLES IN OTHER GLOBULAR CLUSTERS}

In this appendix we show the CV and CV candidates in the core-collapsed clusters NGC 6397 and NGC 6752 that were used in the comparison with the CV population in 47 Tuc (Section 5.

Table C1. CVs and CV candidates in NGC 6397 which have proper motions that are consistent with that of the cluster. The information was taken from Cohn et al. (2010) and Bogdanov et al. (2010).

\begin{tabular}{cccccc}
\hline \hline NGC 6397 & & & & & \\
\hline Source & R.A. & Decl (J2000) & r (') & $\begin{array}{c}L_{X(0.5-6 \mathrm{keV})}{ }^{*} \\
\left(10^{30} \mathrm{ergs} \mathrm{s}^{-1}\right)\end{array}$ & Type \\
\hline U7 - CV10 & $17: 40: 52.832$ & $-53: 41: 21.77$ & 1.81 & 4.8 & CV \\
U10 - CV6 & $17: 40: 48.978$ & $-53: 39: 48.62$ & 1.21 & 22.5 & CV \\
U11 - CV7 & $17: 40: 45.781$ & $-53: 40: 41.52$ & 0.58 & 1.5 & CV \\
U13 - CV8 & $17: 40: 44.084$ & $-53: 40: 39.17$ & 0.33 & 2.9 & CV \\
U17 - CV3 & $17: 40: 42.651$ & $-53: 40: 19.30$ & 0.17 & 141.8 & CV \\
U19 - CV2 & $17: 40: 42.306$ & $-53: 40: 28.70$ & 0.02 & 111.2 & CV \\
U21 - CV4 & $17: 40: 41.830$ & $-53: 40: 21.37$ & 0.13 & 52.0 & CV \\
U22 - CV5 & $17: 40: 41.701$ & $-53: 40: 29.00$ & 0.07 & 2.9 & CV \\
U23 - CV1 & $17: 40: 41.597$ & $-53: 40: 19.30$ & 0.18 & 92.8 & CV \\
U25 - CV13 & $17: 40: 41.237$ & $-53: 40: 25.79$ & 1.0 & 1.3 & CV? \\
U60 - CV9 & $17: 40: 47.807$ & $-53: 41: 28.40$ & 1.30 & 0.25 & CV \\
U61 - CV12 & $17: 40: 45.223$ & $-53: 40: 28.60$ & 0.45 & 2.0 & CV? \\
U80 - CV14 & $17: 40: 46.455$ & $-53: 41: 56.50$ & 1.60 & 0.4 & CV? \\
U83 - CV15 & $17: 40: 49.615$ & $-53: 40: 43.02$ & 1.13 & 0.1 & CV? \\
\hline
\end{tabular}

* Luminosities in the 0.5-6 keV band were calculated by taking the fluxes from Bogdanov et al. (2010), and assuming a distance to NGC 6397 of $2.2 \mathrm{kpc}$. Errors are not provided by the authors.

Table C2. CVs and CV candidates in NGC 6752 which have proper motions that are consistent with that of the cluster, the information was taken from Lugger et al. Lugger et al. (2017) and Forestell et al. (2014).

\begin{tabular}{|c|c|c|c|c|c|}
\hline \multicolumn{6}{|c|}{ NGC 6752} \\
\hline Source & R.A. & Decl (J2000) & $\mathrm{r}\left({ }^{\prime}\right)$ & $\begin{array}{l}L_{X(0.5-6 \mathrm{keV})^{*}} \\
\left(10^{30} \mathrm{ergs} \mathrm{s}^{-1}\right)\end{array}$ & Type \\
\hline CX1 & 19:10:51.138 & $-59: 59: 11.92$ & 0.17 & $259.8_{-17.4}^{+17.4}$ & $\mathrm{CV}$ \\
\hline $\mathrm{CX} 2$ & 19:10:56.005 & $-59: 59: 37.33$ & 0.73 & $126.4_{-11.9}^{+12.4}$ & $\mathrm{CV}$ \\
\hline CX4 & $19: 10: 51.586$ & $-59: 59: 01.73$ & 0.08 & $88.6_{-11.2}^{+11.9}$ & $\mathrm{CV}$ \\
\hline CX5 & $19: 10: 51.414$ & $-59: 59: 05.18$ & 0.09 & $101.6_{-12.1}^{+11.2}$ & $\mathrm{CV}$ \\
\hline CX6 & $19: 10: 51.505$ & $-59: 59: 27.10$ & 0.38 & $42.3_{-6.2}^{+6.5}$ & CV? \\
\hline CX7 & $19: 10: 51.511$ & $-59: 58: 56.85$ & 0.15 & $68.7_{-9.2}^{+9.4}$ & $\mathrm{CV}$ \\
\hline CX9 & $19: 10: 51.766$ & $-59: 58: 59.25$ & 0.10 & $21.5_{-5.0}^{+6.2}$ & CV? \\
\hline CX13 & 19:10:40.610 & $-60: 00: 05.91$ & 1.76 & $5.3_{-1.0}^{+1.2}$ & $\mathrm{CV}$ \\
\hline CX21 & $19: 10: 49.516$ & $-59: 59: 43.16$ & 0.72 & $2.9_{-0.8}^{+0.9}$ & CV? \\
\hline CX23 & $19: 10: 52.546$ & $-59: 59: 04.38$ & 0.06 & $2.1_{-0.7}^{+0.8}$ & CV? \\
\hline CX24 & $19: 10: 52.670$ & $-59: 59: 03.21$ & 0.07 & $2.1_{-0.7}^{+0.8}$ & CV? \\
\hline CX25 & $19: 10: 51.957$ & $-59: 58: 40.55$ & 0.40 & $1.6_{-0.6}^{+0.8}$ & CV? \\
\hline CX32 & $19: 10: 54.137$ & $-59: 59: 11.04$ & 0.28 & $1.05_{-0.5}^{+0.6}$ & CV? \\
\hline CX35 & $19: 10: 52.165$ & $-59: 59: 16.73$ & 0.20 & $0.8_{-0.4}^{+0.6}$ & CV? \\
\hline CX36 & $19: 10: 49.585$ & $-59: 58: 26.41$ & 0.71 & $0.7_{-0.4}^{+0.6}$ & $\mathrm{CV}$ \\
\hline
\end{tabular}

\footnotetext{
* Luminosities in the $0.5-6 \mathrm{keV}$ band were calculated by multiplying the $0.5-8 \mathrm{keV}$ band luminosities given in Forestell et al. (2014) by
} the appropriate factor (0.95) assuming a $2 \mathrm{keV}$ bremsstrahlung model. 